\title{
A feasibility study about the use of vector tomography for the reconstruction of the coronal magnetic field
}

\author{
Dissertation \\ zur Erlangung des Doktorgrades \\ der Mathematisch-Naturwissenschaftlichen Fakultäten \\ der Georg-August-Universität zu Göttingen
}

vorgelegt von

Maxim I. Kramar

aus Witebsk/Belarus

Göttingen 2005 


\section{Bibliografische Information Der Deutschen Bibliothek}

Die Deutsche Bibliothek verzeichnet diese Publikation in der Deutschen Nationalbibliografie; detaillierte bibliografische Daten sind im Internet über http: //dnb. ddb. de abrufbar.

D7

Referent: Prof. Dr. F. Kneer

Korreferent: Prof. Dr. E. Marsch

Tag der mündlichen Prüfung: 19. September 2005

Copyright (C) Copernicus GmbH 2006

ISBN 3-936586-46-2

Copernicus GmbH, Katlenburg-Lindau

Druck: Schaltungsdienst Lange, Berlin

Printed in Germany 


\section{Contents}

$\begin{array}{ll}\text { Summary } & 5\end{array}$

1 Introduction $\quad 7$

2 Scalar Field Tomography $\quad 13$

2.1 Formulation of the scalar field tomography problem . . . . . . . . . . 13

2.2 Matrix formulation of the scalar field tomography problem . . . . . . . 15

2.3 Singular Value Decomposition method . . . . . . . . . . . . . . . . . 16

2.4 Regularization . . . . . . . . . . . . . . . . . 16

3 Vector Tomography 19

3.1 The formulation of the vector field tomography problem . . . . . . . . 19

3.2 Vector tomography for the LOS projection data . . . . . . . . . . . . 20

3.3 Matrix formulation of the vector tomography problem . . . . . . . . . . 22

3.4 Special regularization for coronal vector tomography . . . . . . . . . . 22

4 The possible effects used for deriving the magnetic field 25

4.1 Zeeman-effect . . . . . . . . . . . . . . . . 25

4.2 Hanle-effect . . . . . . . . . . . . . . . . . . 26

4.3 Faraday-effect . . . . . . . . . . . . . . . . . 28

5 The line formation of magnetically sensitive lines 31

5.1 Stokes vector . . . . . . . . . . . . . . . 31

5.2 Radiative Transfer for the Polarized Radiation . . . . . . . . . . . . . . 32

5.3 Statistical-equilibrium equation . . . . . . . . . . . . . . . . 35

5.4 The role of the collisions . . . . . . . . . . . . . . 37

5.5 Photo-excitation . . . . . . . . . . . . . . . 37

5.6 Density matrix in irreducible tensor representation . . . . . . . . . . . . . 39

5.7 The non-coherence approximation . . . . . . . . . . . . . . 40

5.8 Weak field approximation . . . . . . . . . . . . . . . . . 40

5.9 Magnetograph formula . . . . . . . . . . . . . . . . . . 43

5.10 Emission line $10747 \AA$ of the Fe XIII . . . . . . . . . . . . . . 44

5.11 Emission line $5303 \AA$ of the $\mathrm{Fe} \mathrm{xIV} \ldots \ldots \ldots$. . . . . . . . . . . . . . . . . . . . 46

5.12 Influence of the alignment factor . . . . . . . . . . . . . . . 49 
6 Test simulations $\quad \mathbf{5 1}$

6.1 The coordinate system . . . . . . . . . . . . . . . 51

6.2 Discretization of the divergence operator . . . . . . . . . . . . 51

6.3 Discretization of the line-of-sight integration . . . . . . . . . . . 52

6.4 Magnetic field configuration for the test calculations . . . . . . . . . . 53

6.5 Reconstruction based on the Zeeman-effect data . . . . . . . . . . . . . . 54

6.6 Reconstruction based on the Hanle-effect data . . . . . . . . . . . . . . . 59

6.7 Comparison of the Hanle- and Zeeman-effect solutions . . . . . . . . . . 61

6.8 Reconstruction based on the Hanle-effect: Zeeman-effect solution as initial field . . . . . . . . . . . . . . . . . . . 64

$\begin{array}{lll}7 & \text { Conclusion } & 67\end{array}$

$\begin{array}{ll}\text { Outlook } & 71\end{array}$

$\begin{array}{ll}\text { A Appendix } & \mathbf{7 3}\end{array}$

A.1 Potential field approximation . . . . . . . . . . . . . . . 73

A.2 Force-free field reconstruction . . . . . . . . . . . . . . . 74

A.3 Michelson Doppler Imager (MDI) _ . . . . . . . . . . . . . 75

A.4 Spherical tensor $\mathcal{T}$ for polarimetry of M1 transitions . . . . . . . . 77

A.5 Wigner symbols . . . . . . . . . . . . . . . . 78

A.5.1 Wigner $3-j$ symbol $\ldots \ldots \ldots . \ldots . \ldots 78$

A.5.2 Wigner $6-j$ symbol . . . . . . . . . . . . . . . . 79

A.5.3 Wigner $9-j$ symbol . . . . . . . . . . . . 80

A.6 List of analyzed reconstructions _. . . . . . . . . . . . . 82

A.7 Cross sections of the reconstructed field . . . . . . . . . . . . 83

$\begin{array}{ll}\text { Bibliography } & 107\end{array}$

$\begin{array}{lr}\text { Acknowledgements } & 115\end{array}$

$\begin{array}{ll}\text { Curriculum Vitae } & 117\end{array}$ 


\section{Summary}

The magnetic field contains the dominant energy per unit volume in the solar corona and therefore plays an important role in most coronal phenomena. But until now, no direct measurement of the magnetic field vector distribution in the corona could be made. Models of the coronal magnetic field rely almost enterely on extrapolations of photospheric magnetic field observations. Some indirect information about the coronal magnetic field, however, can be obtained using the Faraday, longitudinal Zeeman or Hanle effects on emissions at magnetically sensitive coronal transition lines. The Faraday and longitudinal Zeeman effects provide the line-of-sight component of the magnetic field integrated over the line-of-sight. Polarimetric measurements of the Hanle effect yields information about the magnetic field orientation integrated along the line of sight.

In this thesis, we investigate whether a tomographic reconstruction based on these observations allow us to obtain a reliable model of the vector magnetic field in the whole solar corona. The inversion problem is strongly ill-posed. To improve the condition of the inversion problem we use the fact that the magnetic field has to satisfy $\nabla \cdot \boldsymbol{B}=0$ as an additional regularization constraint. The use of this constraint, however, may require additional solar surface magnetogram data as boundary condition. With the help of this constraint, we show that it is possible to reconstruct both the strength and direction of the magnetic field from the mentioned above observations. The reconstructed field contains details, which cannot be obtained with a traditional extrapolation of the photospheric surface field measurements.

The inversion code based on the effects mentioned above has been developed. The code is tested using simulated data of the longitudinal Zeeman and Hanle effects including some artificial noise. The magnetic field configuration is chosen to consist of two parts: a mean dipole field component and non-potential field component induced by a circular current in the corona. The tomographic inversion of the simulated data allows us to reconstruct the potential and the non-potential component of the field, while a traditional potential field approximation reconstructs only the main dipole field component. 



\section{Introduction}

The corona is the outermost part of the Sun's atmosphere. It is bounded below at $\sim$ $2.5 \mathrm{Mm}$ above the solar surface by the thin $(\sim 500 \mathrm{~km})$ transition region within which the plasma temperature rises from chromospheric values of below $10^{4} \mathrm{~K}$ to typical coronal temperatures of above $10^{6} \mathrm{~K}$. The solar corona is structured by the coronal magnetic field which is rooted at the solar surface and is partially open to the heliosphere. The outer boundary of the corona is not precisely defined. Its outer boundary may be placed at a distance of $\sim 2-3 R_{\odot}$ above the solar surface where the magnetic field lines are dragged out by the solar wind and bent into radial direction.

The solar corona consists of a hot $\left(>10^{6} \mathrm{~K}\right)$, highly ionized and very low density plasma $\left(\lesssim 10^{10} \mathrm{~cm}^{-3}\right)$. The highest temperature of the coronal plasma is achieved in regions with closed magnetic field lines where the plasma is confined and cannot escape into the heliosphere. The reason for its high temperature is still uncertain but most explanations for the coronal heating mechanism involve the coronal magnetic field (Zirker 1993; Ulmschneider 1998; Erdelyi 2004).

The bulk motion of the coronal plasma as a fluid is governed by the pressure gradient, gravity and magnetic Lorentz force. The ratio of the first two forces can be expressed by the ratio of the pressure scale height $H$ to a typical length scale $L$ over which the pressure varies. Here, $H=k_{B} T / m_{\mathrm{H}} g_{\odot}$ where $k_{B}$ is the Boltzmann constant, $T$ is the coronal temperature, $m_{\mathrm{H}}$ is the mass of a proton, the dominant ion in the corona, and $g_{\odot}$ is the Sun's gravitational acceleration. Perpendicular to the field lines, typically $L \ll H \sim$ $50 \mathrm{Mm}$, so that gravity often plays a minor role. The ratio of the pressure force to the Lorentz force $\boldsymbol{J} \times \boldsymbol{B}$ is expressed by the parameter $\beta=2 \mu_{0} p / B^{2}$, where $p$ is the thermal pressure, $\boldsymbol{B}$ is the magnetic field vector, $\boldsymbol{J}$ is the electric current density vector, and $\mu_{0}$ is the magnetic permeability. In the inner corona from the chromosphere up to $\sim 1.5 R_{\odot}$, (and sometimes higher) the plasma- $\beta$ mostly is less than unity (Gary 2001). Therefore, the coronal magnetic field is strong enough to effectively dominate the plasma motion. It can therefore be considered as the main driving force of most plasma phenomena occurring in the inner corona. To understand the physics of the corona, a detailed knowledge of the coronal magnetic field is therefore absolutely essential.

Unfortunately, direct magnetic field measurements in the corona are extremely difficult. The majority of solar magnetic field measurements in the past have been taken in the photosphere. A standard way to estimate the field in the corona is then to extrapolate the radial photospheric magnetic field component using the potential field or more sophisticated model approximations. But the potential field extrapolation does not take into account any electric current in the corona and, therefore, misses an important part of the physics. Since the potential magnetic field is the field with the minimum energy for a given photospheric radial boundary condition (Sakurai 1989), it cannot account for 
dynamical processes such as eruptions and flares, where very probably magnetic energy is converted into plasma kinetic energy. Indeed, soft X-ray observations of active regions often show a non-potential structure of the magnetic field (Jiao et al. 1997). Observations of the horizontal surface field by vector magnetographs have been used to estimate radial currents in the photosphere, part of which probably flows along field lines out into the corona (Hagyard \& Pevtsov 1999). More realistic models of the coronal field can be obtained by applying the force-free approximation which neglects pressure and gravity forces (Cuperman et al. 1990; Flyer et al. 2004). This method consists in solving a boundary value problem for the equations $(\nabla \times \boldsymbol{B}) \times \boldsymbol{B}=0$ and $\nabla \cdot \boldsymbol{B}=0$, using the radial and horizontal magnetic field components measured at the photospheric level as boundary values. The main difficulties here are: the procedure is numerically ill-posed, and the results are less reliable with increasing distance from the surface (Demoulin et al. 1992).

The measurements used for the determination of the line-of-sight (LOS) component of the magnetic field at the photosphere are based on the Zeeman effect. At optical wavelengths this method is not sensitive enough to be used for coronal measurements, because the coronal magnetic field strength $(\sim 10 \mathrm{G})$ is relatively small and the temperature $\left(\sim 10^{6} \mathrm{~K}\right)$ in the corona is very high so that the Zeeman splitting is much smaller than the thermal broadening of the line.

Polarimetric coronograph observations of coronal emission lines have been used to estimate the orientation of the coronal magnetic field in the plane of the sky (POS) through the Hanle effect for emission lines from forbidden atomic transitions. The possibility of making use of the Hanle effect to derive the coronal magnetic field from the polarimetric measurements was first investigated by Charvin(1965) and was further developed by many authors (House 1974,1977; House et al. 1982; Sahal-Brechot 1974a,b, 1977; Cassini \& Judge 1999). An example of such an observations is shown in Fig. 1.1a.

Landi Degl'Innocenti \& Landi Degl'Innocenti (1973), Landi Degl'Innocenti (1982) and later Casini \& Judge (1999) proposed and investigated the possibility to use spectropolarimetric observations at infrared wavelengths to derive a longitudinal magnetic field estimate in the corona from the longitudinal Zeeman effect. Promising coronagraph measurements of this kind have only recently been achieved for infrared coronal lines (Lin et al. 2000, 2004). Although these measurements were restricted to heights below $0.45 R_{\odot}$ above an active region, they demonstrate that the measurement technique has the potential to be applied to the whole corona. Currently, a precision of $\pm 1 \mathrm{G}$ is obtained with these observations after 70 minutes of integration (for Stokes- $V$ spectrum).

Both the coronal Hanle effect observations and the coronal Zeeman effect measurements are effectively LOS integrations through the corona because the respective coronal lines are optically thin. The measurements are therefore not localized and their interpretation in terms of magnetic flux densities is not straightforward. In the present thesis we want to explore to which extent these data provide sufficient information to allow a reconstruction of the three-dimentional (3D) magnetic field in the corona. It is not clear that the information is sufficient: a 3D vector field locally has three degrees of freedom (i.e., the three vector components), while the Zeeman and Hanle effect observations provide only a single scalar value (the projection of the vector along the LOS integrated over the LOS for the Zeeman effect) or two scalar values (polarization degree and orientation of the polarization plane integrated over the LOS for the Hanle effect). 
The inversion of LOS integrated information to a local 3D distribution is called a tomographic inversion. In the past, the tomography technique has been applied in solar physics only for the reconstruction of a scalar field, the electron and ion densities. The principle of scalar field tomography is based on measuring the LOS integral of the absorption or emissivity through the region to be investigated. The observations must be performed from different positions to be able to resolve the 3D structure. The application of scalar field tomography in solar physics has been investigated before by several authors (Davila, 1994; Davila and Thompson, 1992; Zidowitz 1999; Frazin 2000; Frazin \& Janzen 2002).

Since we want to reconstruct a vector field, we employ an extension of classical scalar

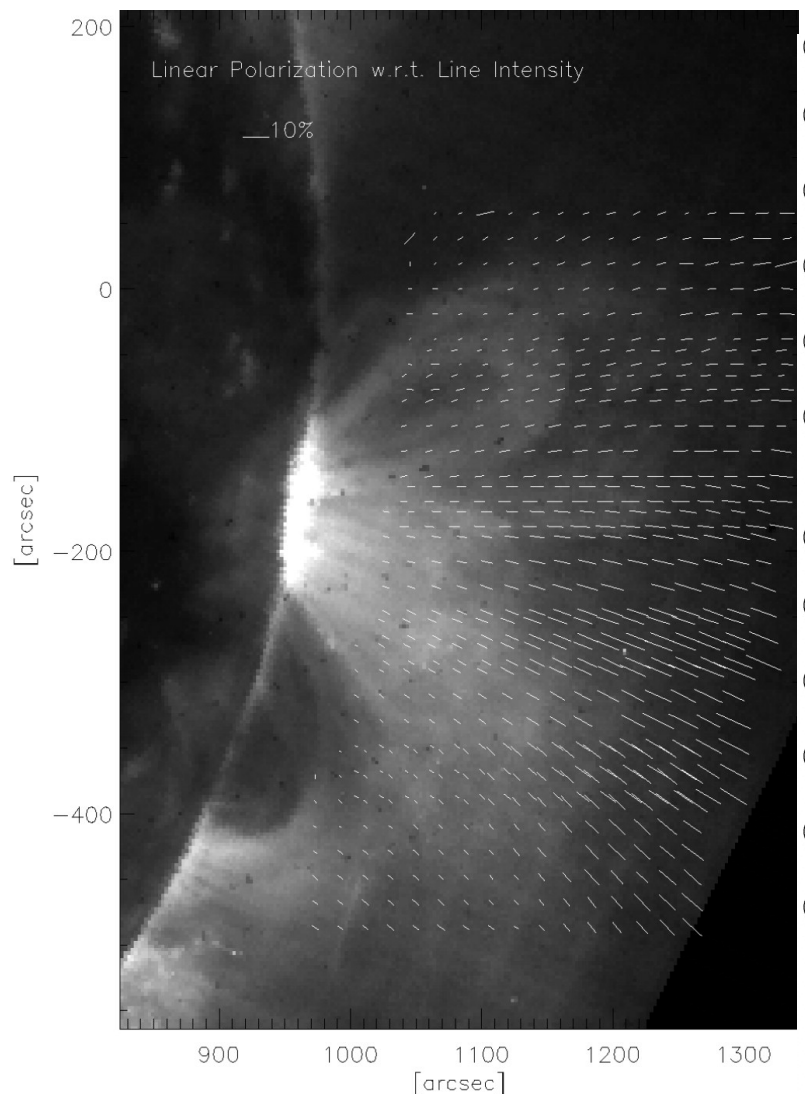

(a)

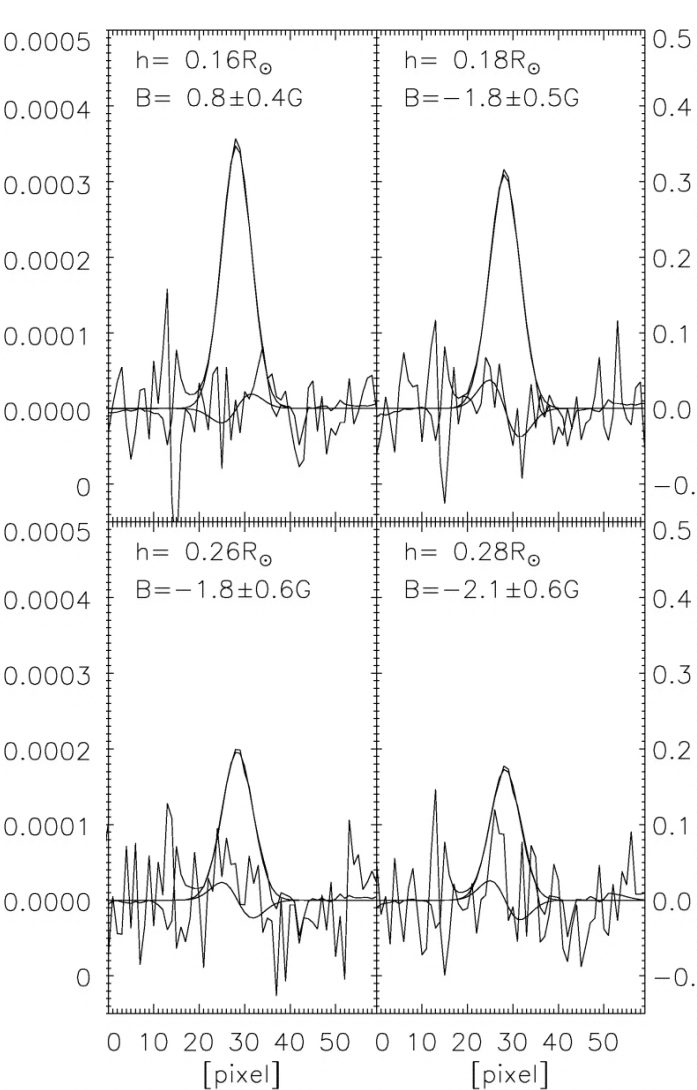

(b)

Figure 1.1: An example of the spectropolarimetric observations of the coronal line $\lambda=10747 \AA$ emitted from Fe xIII taken from Lin et al. (2004). The picture on the panel (a) shows the measurements of the Hanle effect as short lines which represent the orientation and, by their length, the degree of the line's linear polarization. The measurements were made above the active region NOAA AR 581 shown in the EUV image (EIT, $\lambda=284 \AA$ of $\mathrm{Fe} \mathrm{xv}$ ) onto which the polarization data is superposed. The graphics on the panel (b) show the fitted Stokes- $V$ profiles due to the longitudinal Zeeman effect (antisymmetric smooth lines, left-side scale) and fitted Stokes- $I$ profiles (lines having biggest symmetric peak, right-side scale) above the active region. The values of the projection of the magnetic field vector on the LOS which best fits the lines shapes are shown in the inserts. These values were calculated by the standard magnetograph formula without correction for an alignment factor different from unity (see section 5.9). 
field tomography, namely vector field tomography (Sparr \& Strahlen 1998; Osman \& Prince 1997). A variant of vector tomography is used in astrophysical observations, sometimes called Doppler tomography, to derive plasma flow around binary systems and accretion disks (see Boffin et al. (2001) for a review), for the plasma diagnostic in the laboratory plasmas (Howard 1996; Fuchs \& Pickalov 1998). To our knowledge, this thesis is the first investigation on whether vector tomography can be used in solar physics for reconstruction of coronal magnetic fields. The tomography of vector fields is fundamentally more difficult than scalar field tomography. While the latter is mildly ill-posed (Natterer 1986), Norton (1989) showed that a whole class of vector fields may be invisible to certain types of observations. Hence, the inversion problem of vector field tomography has to cope with an extended null-space. For example, for the 2D vector field, Norton (1989) showed that the irrotational field component cannot be reconstructed from the LOS projected data.

In the present thesis, we consider the possibility of a reconstruction of the coronal magnetic field by tomographic technique based on possible coronagraph observations of the Hanle and Zeeman effects. A prerequisite for a succesful reconstruction is a quantitative describtion of these effects for the emission lines observed. In this work we focus our considerations where quantitative expressions are needed on two coronal emission lines: $\lambda=5303 \AA$ of Fe xIv, for which many of the Hanle effect observations were made (Arnaud 1982a,b; Querfeld \& Smartt 1984; ), and $\lambda=10474 \AA$ of Fe XIII for which both the Hanle effect (Arnaud \& Newkirk 1987; Habbal et al. 2001) and the longitudinal Zeeman effect observations are in promising progress (Lin et al. 2000, 2004). It is assumed that the observations are made repetitively for one solar rotation. The problems which arise due to the non-stationarity of the coronal magnetic field during the measurement sequence are ignored here.

The $5303 \AA$ line of Fe XIV (green line) is the brightest forbidden emission line in the visible coronal spectrum. The line was discovered by Young and Harkness during the 1869 total solar eclipse, and identified later by Grotrian (1939) and Edlen (1943). A large progress in intensity observations of the line was reached by the launch of the SOHO spacecraft with LASCO coronagraph on board (Brueckner et al. 1995; Schwenn et al. 1997; Inhester et al. 1999). A specially designed coronameter to measure the green line polarization has been built at Pic-du-Midi observatory (Charvin 1971; Arnaud 1982a). For the Hanle effect application it is sufficient to perform line-integrated polarimetric measurements over the line profile. It is not necessary to use spectropolarimetry.

The coronal emission line at $10747 \AA$ was first observed by B. Lyot (Lyot 1939) in 1936. The first measurements of the polarization of the line were made by Eddy and Malville (1967) during the 1965 eclipse. Later, the Coronal Emission Line Polarimeter (KELP) was constructed by the High Altitude Observatory (HAO) and located at the Sacramento Peak Observatory to provide more precise measurements (Querfeld \& Elmore 1976; Querfeld \& Smartt 1984). Lin et al. (2000, 2004) have achieved considerable progress in the spectropolarimetric measurements of the line (see Fig. 1.1b) so that they could estimate the longitudinal field strength from the Stokes- $\mathrm{V}$ polarization component.

The goal of the thesis is to investigate the possibility to apply vector field tomography based on the polarimetric and spectropolarimetric measurements of the lines mentioned above in order to reconstruct the coronal magnetic field.

The thesis is organized as follows. In the second and third chapters the fundamentals 
of scalar and vector field tomography are reviewed. The observational geometry is defined there. Special emphasize is devoted to the question of how an inversion effectively can be improved by regularization (section 2.4), and to the theoretical limitations of vector tomography (section 3.2). A special regularization operator described in section 3.4 will be used in an attempt to overcome these limitations.

The inversion of the LOS integrated data requires a quantitative understanding of how the observed signal is formed. In our case, we need quantitative expressions for the formation of the coronal lines at $\lambda=5303$ and $10747 \AA$. The Zeeman and Hanle effects are qualitatively reviewed in chapter 4 . Chapter 5 is devoted to the description of quantitative expressions for the observed polarimetric and spectropolarimetric data in terms of the magnetic field. These expressions are then used in developing an inversion code for the data and in demonstrating its capabilities in chapter 6 . The results of our model reconstructions are summarized in the conclusion chapter. In a final outlook we point out the potential of our new method and how furthers improvements can be made. 



\section{Scalar Field Tomography}

In this chapter the basics of scalar tomography are described. As for many ill-posed problems, the tomography inversion is often stabilized by regularization which is also illustrated in this chapter. Although currently in the inversion program we do not use the traditional form of the regularization presented in this chapter, it can be incorporated into the vector field reconstruction procedure too. Instead we will modify the regularization approach for our purposes as described in chapter 3 .

\subsection{Formulation of the scalar field tomography problem}

For wavelength for which the corona is optically thin, the radiation coming from the corona is a LOS integral of the emissivity in the observed direction. Therefore, it is impossible to reconstruct the spatial distribution of the emissivity from a single (in geometric sense) measurement or projection. The solution space is reduced if we have measurements from many different view points. The reconstruction based on the observations of an object from different view angles is essential for tomography. The possibility of the reconstruction of a function from its projections was firstly studied by Radon (1917). Several decades later, this purely mathematical research formed the basis for the tomography method which was developed for the reconstruction of the X-ray absorption coefficient in human bodies. The first experimental X-ray tomographic scanner was made

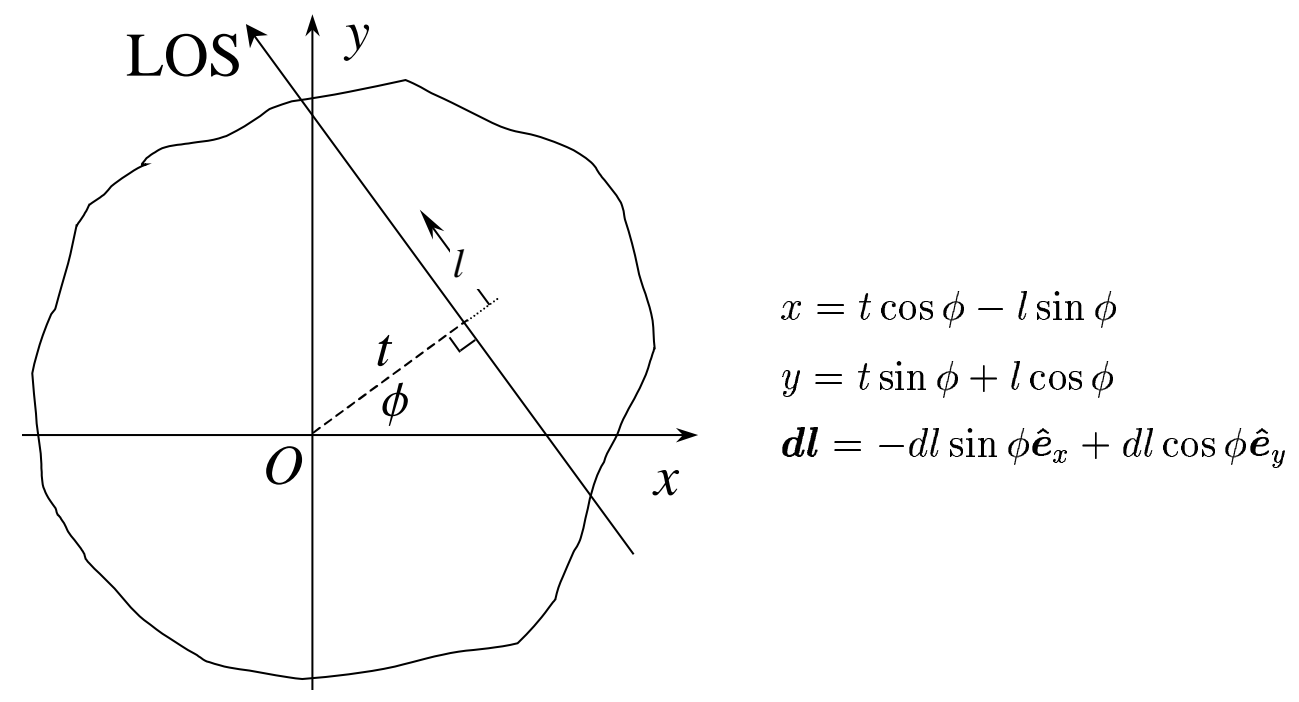

Figure 2.1: The 2D slice with the line-of-sight (LOS) in the scalar field tomography. 
by Hounsfield $(1972)$, and Cormack $(1963,1964)$ independently discovered some of the algorithms for the reconstruction. The both authors received the Nobel prize for their investigations in 1979. Today, tomography is used in many of the fields: medicine, material structure testing, geophysics, thermonuclear synthes investigations (TOKAMAK reactors), astrophysics (Doppler tomography for the accretion disks) (Boffin 2001). In solar coronal physics, the use of tomography was first proposed by Wilson (1976) and independently by Davila $(1992,1994)$. Recently, the method was applied for reconstruction of the 3D electron density distribution over the corona from 2.4 to $6.0 R_{\odot}$ (Frazin 2000; Frazin \& Janzen 2002).

Since the distance between the Sun and the Earth orbit is much greater than size of investigated volume of the corona ( $\sim 4 R_{\odot}$ in diameter in our investigations), it is possible to assume that the rays related to all the pixels of one image are parallel (parallel beam geometry).

Let us make the following two assumptions:

1) The corona is stationary during at least one solar rotation, that does not cause to an big error during solar minimum, and rotates together with Sun. So, it is possible to make measurements from the Earth during solar rotation instead of observations from many of spacecrafts.

2) The inclination of the Sun's rotation axis with respect to the ecliptic plane $\left(7.26^{\circ}\right)$ is neglected, i.e. we assume that the rotation axis is perpendicular to the line connecting the Sun and the Earth. Then, we can divide the investigated volume into plane-parallel slices in such way, that each of them is perpendicular to the rotation axis, and rays that penetrate one slice do not intersect any other slice; i.e. slices are decoupled, and can be processed independently. This assumption can be avoided twice per year.

So, to reconstruct a 3D configuration of a scalar field $\varepsilon(\boldsymbol{r})$, it is possible to split the investigated volume in plane-parallel slices with normals along the rotation axis and deal with the 2D problem for every slice separately. Let us consider one slice (Figure 2.1). A single data point defined by the observing angle, $\phi$, and distance of the ray from the origin, $t$, can be described by equation

$$
I(\phi, t)=\int_{\operatorname{LOS}_{\phi, t}} \varepsilon(x, y) d l=\int_{\operatorname{LOS}_{\phi, t}} \varepsilon(t \cos \phi-l \sin \phi, t \sin \phi+l \cos \phi) d l .
$$

The Fourier transform of $I(\phi, t)$ with respect to image position, $t$, is

$$
\begin{array}{r}
\tilde{I}(\phi, \rho)=\int I(\phi, t) e^{-i \rho t} d t= \\
=\int\left[\int_{\operatorname{Los}_{\phi, t}} \varepsilon(t \cos \phi-l \sin \phi, t \sin \phi+l \cos \phi) e^{-i \rho t} d l\right] d t= \\
=\iint \varepsilon(x, y) e^{-i \rho(x \cos \phi+y \sin \phi)} d x d y=\tilde{\varepsilon}(\rho \cos \phi, \rho \sin \phi)=\tilde{\varepsilon}(u, v)
\end{array}
$$

Equation (2.2) is the central slice theorem of scalar tomography (Natterer 1986; Kak \& Slaney 1987). The right hand side in (2.2) is the two-dimensional Fourier transform of the scalar field function. Therefore, applying the inverse two-dimensional Fourier transform 
to $\tilde{I}$ we reconstruct $\varepsilon(x, y)$ :

$$
\varepsilon(x, y)=\iint \tilde{\varepsilon}(u, v) e^{i(u x+v y)} d u d v=\iint \tilde{I}(\phi, \rho) e^{i \rho(x \cos \phi+y \sin \phi)} \mathcal{J}(\rho, \phi) d \rho d \phi
$$

where

$$
\mathcal{J}(\rho, \phi)=\left|\begin{array}{ll}
\frac{\partial u}{\partial \rho} & \frac{\partial u}{\partial \phi} \\
\frac{\partial v}{\partial \rho} & \frac{\partial v}{\partial \phi}
\end{array}\right|=\left|\begin{array}{cc}
\cos \phi & -\rho \sin \phi \\
\sin \phi & \rho \cos \phi
\end{array}\right|=\rho
$$

is Jacobian determinant of the variable transform $(u, v) \rightarrow(\rho, \phi)$. This way of reconstruction is call the Fourier method. Applying this method for every plane, we can reconstruct the scalar function $\varepsilon(\boldsymbol{r})$ in all three dimensions. The method, however, involves the re-sampling from polar to rectangular coordinates which leads to considerable interpolation. This makes the solution very noisy. In practice, other numerical methods are used. However, the Fourier method is useful for analytical investigations of reconstruction possibilities in principal. Especially, it allows one to proof that a reconstruction from the projections is possible.

The corona extends to the Earth's orbit and further. But we concentrate on measurements only of the part of the corona up to several solar radii. This is called interior problem, and this problem has no unique solution. Only bounded problems have a unique solution (Natterer 1986). Therefore, we need to introduce an outer boundary, so that regions outside of the bounded domain do not make any contribution to the data. This assumption is close to reality because the coronal mass density and strength of magnetic field decrease with distance from the sun very rapidly.

Observations of the solar corona have one more special feature: on some slices, the central part of the image is occulted, i.e. we have data only for $t^{2}>R_{\odot}^{2}-h^{2}\left(h \leq R_{\odot}\right)$, where $h$ is the distance from the ecliptic plane to the reconstructing slice. This is called the exterior problem in tomography, and it is uniquely solvable too (Natterer 1986).

\subsection{Matrix formulation of the scalar field tomography problem}

Discretizing the LOS integral (2.1) we obtain a set of algebraic equations which can be represented by the matrix equation

$$
\mathbf{A} \cdot \mathbf{X}=\mathbf{Y}
$$

Here, the elements $x_{j}$ of the column vector $\mathbf{X}$ contain the values of $\varepsilon$ in the grid cells with index $j=1, \ldots, n$, and $y_{i}$ is the data value for the $i$-th ray, where index $i=1, \ldots, m$ counts both the discrete view angle $\phi$ and pixel position $t$. The matrix element $a_{i j}$ represents the LOS projection of volume element $j$ onto LOS related to the pixel $i$.

Usually, the matrix $\mathbf{A}$ is rectangular $(m \times n, m \neq n)$ with $\operatorname{rank}(\mathbf{A}) \leq \min (m, n)$. There are two important cases:

1) If $\operatorname{rank}(\mathbf{A})<n$, then the system is underdetermined, and there are infinitely many solutions;

2) If $\operatorname{rank}(\mathbf{A})<m$, then the system is overdetermined, and usually no solutions are possible. 
Direct inversion is formally possible if $m=n=\operatorname{rank}(\mathbf{A})$, which is usually not the case. In all other cases it is possible to find some approximation to the real solution (residual), $\tilde{\mathbf{X}}$, by minimizing the distance between $\mathbf{A} \cdot \mathbf{X}$ and $\mathbf{Y}$ (least-square fitting), i.e.

$$
\tilde{\mathbf{X}}=\operatorname{argmin}_{\mathbf{X}}|\mathbf{A} \cdot \mathbf{X}-\mathbf{Y}|^{2} .
$$

In the underdetermined case, (2.6) yields the solution with the smallest norm, $\min |\tilde{\mathbf{X}}|^{2}$; in the overdetermined case, we have the solution with the smallest data error, $\min |\mathbf{A} \cdot \tilde{\mathbf{X}}-\mathbf{Y}|^{2}$.

\subsection{Singular Value Decomposition method}

One of the methods which is suitable for numerical calculations and allows one to find approximate solutions for both cases listed above is based on the singular value decomposition (SVD) of the matrix A developed by Lanczos (1961).

Any real rectangular matrix ( $m$ rows, $n$ columns) can be decomposed into three matrices:

$$
\mathbf{A}=\mathbf{U} \cdot \boldsymbol{\Sigma} \cdot \mathbf{V}^{\mathrm{T}}
$$

where $\mathbf{U}$ and $\mathbf{V}$ are $m \times n$ and $n \times n$ matrices, respectively, and both have orthogonal columns so that

$$
\mathbf{U}^{\mathrm{T}} \mathbf{U}=\mathbf{1}_{m}, \mathbf{V}^{\mathrm{T}} \mathbf{V}=\mathbf{1}_{n} .
$$

The columns of the matrices $\mathbf{U}$ and $\mathbf{V}$ are the left and right singular eigenvectors of A, respectively. $\Sigma$ is a square $n \times n$ matrix with off-diagonal elements set to be zero and non-negative diagonal elements, $\sigma_{i}$ (with $i=1, \ldots, n$ ), which are the eigenvalues (singular values) of the matrix $\mathbf{A}$ usually ordered with descending magnitude. In the case of $\operatorname{rank}(\mathbf{A})<n$, all the rest $n-\operatorname{rank}(\mathbf{A})$ diagonal elements in $\boldsymbol{\Sigma}$ are set to zero.

The residual of (2.5) is calculated by pseudoinverse of $\mathbf{A}$ :

$$
\tilde{\mathbf{X}}=\left(\mathbf{V} \boldsymbol{\Sigma}^{-1} \mathbf{U}^{\mathrm{T}}\right) \mathbf{Y}
$$

where the elements $\lambda_{i}$ of the inverse matrix $\Sigma^{-1}$ are equal to $1 / \sigma_{i}$. The singular values $\sigma_{i}$ strongly decrease with $i$. Therefore, to avoid a big error in $\lambda_{i}$ for the large $i$, the values of $\lambda_{i}$ corresponding to the small singular values are set to zero, because small $\sigma_{i}$ means that the measurements were insensitive to the structure represented by eigenvector $\boldsymbol{u}_{i}(i$-th column of the $\mathbf{U})$.

For problems which involve integral equations, the left and right singular vectors $\mathbf{u}_{i}$ and $\mathbf{v}_{i}$ tend to an oscillatory behaviour with increasing index $i$, i.e., with decreasing value of $\sigma_{i}$. Therefore, setting these elements to zero (cut-off), the high-frequency components in the solution are damped, i.e., the solution is smoother.

The SVD method is only possible for moderately small $\left(\operatorname{rank}(\mathbf{A}) \lesssim 10^{3}\right)$ matrices. For the high-rank matrices, it is more suitable to use algorithms based on the algebraic iterative techniques.

\subsection{Regularization}

In astrophysics we usually have underdetermined problems. Moreover, data obtained from measurement process are always contaminated by noise. So $\mathbf{Y}=\mathbf{Y}_{0}+\mathbf{E}$, where 
$\mathbf{Y}_{0}$ is the unperturbed data, and $\mathbf{E}$ is a vector containing random noise. Therefore, to stabilize the problem, it is necessary to add more information about the possible solution. One type of additional information can be the property that the residual norm $|\tilde{\mathbf{X}}|$ must be as small as possible. To find a solution in this case, we should minimize $|\mathbf{A} \cdot \mathbf{X}-\mathbf{Y}|^{2}$ and $|\mathbf{X}|^{2}$ together, which is the simpliest form of the regularization method. The regularization method was developed independently by Phillips (1962) and, in a more general way, by Tikhonov (1963). For the problem (2.5) the regularized solution can be found by the minimization:

$$
\tilde{\mathbf{X}}_{\mu}=\operatorname{argmin}_{\mathbf{X}}\left(|\mathbf{A} \cdot \mathbf{X}-\mathbf{Y}|^{2}+\mu|\mathbf{R} \cdot \mathbf{X}|^{2}\right),
$$

where the second term is a general linear regularization term with the regularization matrix $\mathbf{R}$ and regularization parameter $\mu$. If $\mathbf{R}$ is a unit matrix, then we have a zero-order regularization term. If $\mathbf{R} \cdot \mathbf{X}$ represents a finite difference approximation to the first derivative, there is first-oder regularization; and, analogously, when $\mathbf{R} \cdot \mathbf{X}$ represents a finite difference approximation to the second derivative, we have a second-oder regularization, and so on for the higher orders. The general solution of the minimizing problem (2.10) is

$$
\tilde{\mathbf{X}}_{\mu}=\left[\left(\mathbf{A}^{\mathrm{T}} \mathbf{A}+\mu^{2} \mathbf{R}^{\mathrm{T}} \mathbf{R}\right)^{-1} \mathbf{A}^{\mathrm{T}}\right] \mathbf{Y}
$$

The eigenvalues of matrix $\mathbf{A}^{\mathrm{T}} \mathbf{A}$ are $\sigma_{i}^{2}$. Even if they are small or zero for large $i$, the eigenvalues of $\left(\mathbf{A}^{\mathrm{T}} \mathbf{A}+\mu^{2} \mathbf{R}^{\mathrm{T}} \mathbf{R}\right)$ are finite, and the smallest is controlled by $\mu$.

The solution (2.11) depends on the chosen regularization parameter $\mu$. What is the optimal value of $\mu$ ? To answer this question, it is very convenient to use the L-curve method (Miller 1970; Lawson \& Hanson 1974; Hansen 1993). The L-curve is a log-log plot of the residual norm $\left|\mathbf{A} \cdot \tilde{\mathbf{X}}_{\mu}-\mathbf{Y}\right|^{2}$ versus the norm $\left|\mathbf{R} \cdot \tilde{\mathbf{X}}_{\mu}\right|^{2}$ of the regularization term. The curve is often has an "L" shape (Fig. 2.2).

The error $\tilde{\mathbf{X}}_{\mu}-\mathbf{X}_{\mathbf{0}}$ of the regularized solution consists of two components: the perturbation error from the error $\mathbf{E}$ in the data and regularization error due to the regularization of the error-free data component $\mathbf{Y}_{\mathbf{0}}$.

The almost vertical part of the L-curve corresponds to the solutions where the regularization error dominates, i.e. where the solution is very smooth due to the big value

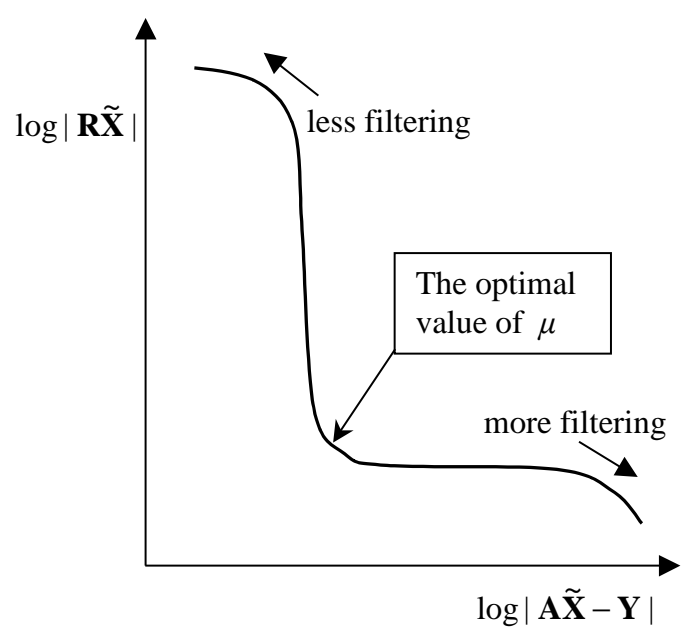

Figure 2.2: The L-curve 
of $\mu$, and therefore the regularization term $\left|\mathbf{R} \cdot \tilde{\mathbf{X}}_{\mu}\right|^{2}$ changes a little with the regularization parameter. The almost horizontal part of the curve corresponds to solutions that are dominated by the data error, and therefore the smoothness of the solution rapidly decreases. The logarithmic scale emphasizes this difference of the vertical and horizontal parts (Hansen 1992; Hansen \& O'Leary 1993). Therefore, it is logical to choose the optimum value of $\mu$ which corresponds to the point closest situated to the corner of the L-curve (Hansen \& O'Leary 1993). It is impossible to find a solution that corresponds to a point below the L-curve. Any regularized solution must be on or above (when it was not enough number of iterations proceeded) this curve. 


\section{Vector Tomography}

The basic introduction to the vector tomography is presented here. The possibility of the reconstruction of the irrotational and solenoidal components of the $3 \mathrm{D}$ vector field are considered from a theoretical point of view. Then, for practical implementation the discrete formulation of the vector tomography problem in a matrix form is introduced. A modified regularization procedure for the vector tomography of the magnetic field is described.

\subsection{The formulation of the vector field tomography prob- lem}

Vector tomography data for optically thin objects is generally expressed by line-of-sight integrals like for scalar field tomography, but instead of a scalar function $\varepsilon(\boldsymbol{r})$ we must reconstruct a vector argument $\boldsymbol{B}(\boldsymbol{r})$ of a scalar function $S$. The observations can be expressed as

$$
I\left(\phi, \theta, x_{p}, y_{p}\right)=\int_{\operatorname{LOS}\left(\phi, \theta, x_{p}, y_{p}\right)} S\left(\boldsymbol{r}, \boldsymbol{B}(\boldsymbol{r}), \hat{\boldsymbol{e}}_{\phi, \theta}\right) d l,
$$

where angles $\phi$ and $\theta$ define the direction $\hat{\boldsymbol{e}}_{\phi, \theta}$ of the LOS in a spherical coordinate system, $x_{p}$ and $y_{p}$ are coordinates of the image pixel in the projection plane (Fig. 3.1). Contrary to scalar field tomography, not only the integration domain but also the integrand, function $S$ in (3.1), depends on the LOS direction.

Depending on the physical effect used in the measurements, the function $S$ can depend on some components of $\boldsymbol{B}$. For example, the longitudinal component, $B_{\|}=\boldsymbol{B} \cdot \hat{\boldsymbol{e}}_{\phi, \theta}$ for the Faraday- and Zeeman-effect, or the direction of the transverse component $\boldsymbol{B}_{\perp}=$ $\boldsymbol{B} \times \hat{\boldsymbol{e}}_{\phi, \theta}$ for the Hanle-effect with respect to the LOS:

$$
\left(\begin{array}{c}
I^{\|} \\
I^{\perp}
\end{array}\right)_{\phi, \theta, x_{p}, y_{p}}=\int_{\operatorname{LOS}\left(\phi, \theta, x_{p}, y_{p}\right)}\left(\begin{array}{c}
S^{\|}\left(\boldsymbol{r}, \boldsymbol{B} \cdot \hat{\boldsymbol{e}}_{\phi, \theta}\right) \\
S^{\perp}\left(\boldsymbol{r}, \boldsymbol{B} \times \hat{\boldsymbol{e}}_{\phi, \theta}\right)
\end{array}\right) d l .
$$

The special expressions for $S^{\|}$and $S^{\perp}$ (Zeeman and Hanle observations) will be derived later in the chapter 5 . Here we will consider simplified, linearized expression $S^{\|}=f(\boldsymbol{r})\left(\boldsymbol{B} \cdot \hat{\boldsymbol{e}}_{\phi, \theta}\right) \equiv \boldsymbol{V}(\boldsymbol{r})$ to derive some theoretical results. Firstly, the linearization is a good approximation for the actual kernel for Faraday- and Zeeman-effect observations. Secondly, the limitations of a reconstruction with the linearized kernel will almost certainly prevail for the non-linear problem. 


\subsection{Vector tomography for the LOS projection data}

The first row of (3.2) connected with $B_{\|}$is known as vectorial ray transform. The tomography problem for the vectorial ray transform is investigated for the case of velocity field of a fluid flow (Sparr \& Strahlen 1998; Osman \& Prince 1997; and references therein). In this case the data for the coronal observations of the longitudinal Zeeman or Faraday effects is expressed by

$$
\begin{gathered}
I^{\|}\left(\phi, \theta, x_{p}, y_{p}\right)=\int_{\operatorname{LOS}\left(\phi, \theta, x_{p}, y_{p}\right)} f(\boldsymbol{r}) \boldsymbol{B}_{\|}(\boldsymbol{r}) \boldsymbol{d} \boldsymbol{\ell}=\int_{\operatorname{LOS}\left(\phi, \theta, x_{p}, y_{p}\right)} \boldsymbol{V}_{\|}(\boldsymbol{r}) \boldsymbol{d} \boldsymbol{\ell}= \\
=\int_{\operatorname{LOS}\left(\phi, \theta, x_{p}, y_{p}\right)}\left(V_{x} \cos \phi \sin \theta+V_{y} \sin \phi \sin \theta+V_{x} \cos \theta\right) d \ell
\end{gathered}
$$

where $f(\boldsymbol{r})$ is some scalar function of position in space (in the case of the Faraday effect, this function is the electron density), and $\boldsymbol{V}=f \boldsymbol{B}$. In the case when the observer positions are confined to the equatorial plane $\theta=\pi / 2$, we can decompose the reconstruction

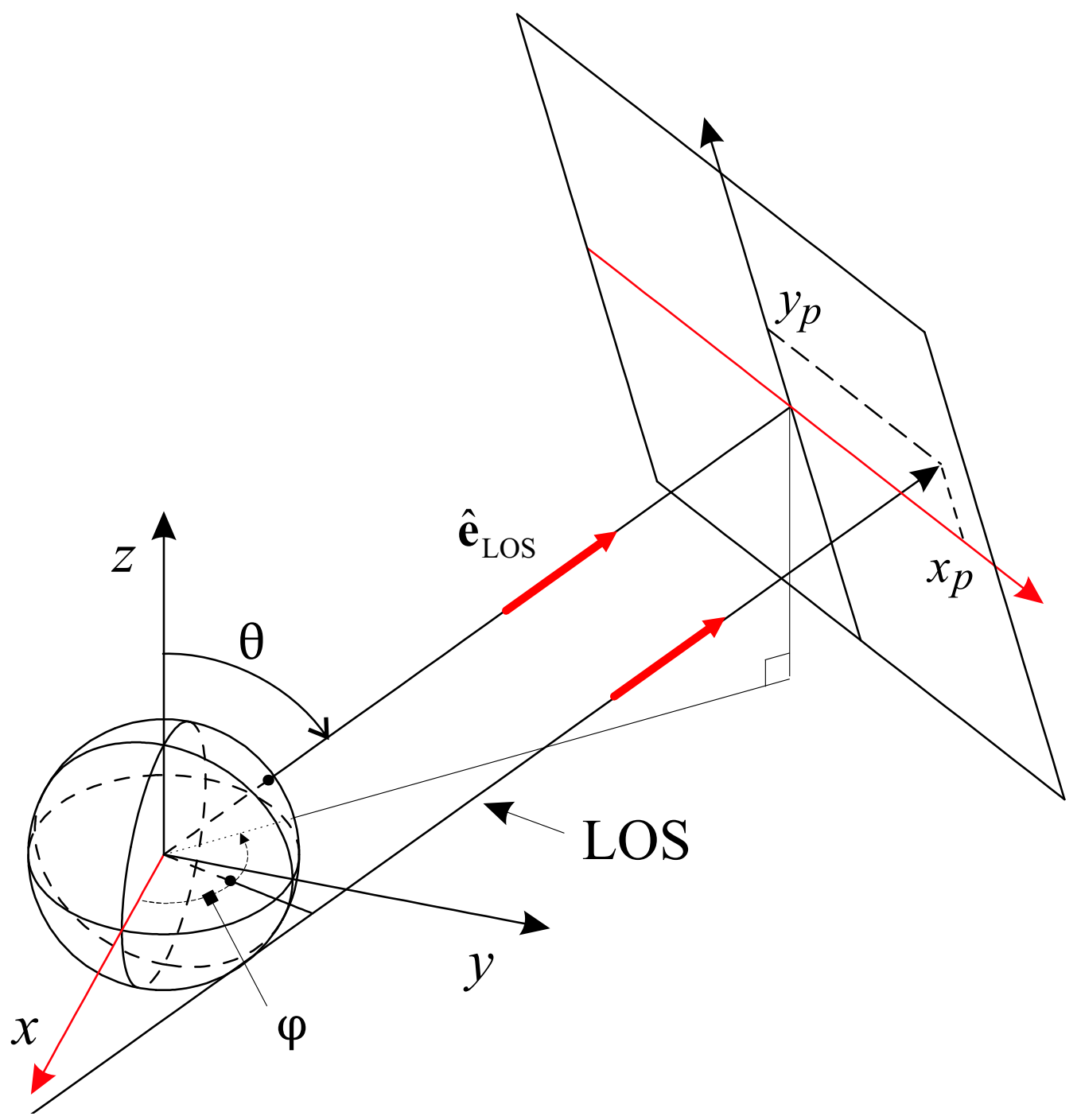

Figure 3.1: The 3D view of the tomography geometry 
domain into plane-parallel slices normal to the $z$-axis. Then, from eq. (3.3) one can see that for the plane-parallel case only two components of the vector field, $V_{x}$ and $V_{y}$, contribute to the data. Therefore, having $I^{\|}$-type data from plane-parallel measurements, it is impossible to fully reconstruct a vector field without additional constraint. This, however, is the situation for most observing space crafts usually located in the plane of the ecliptic.

But even the reconstruction of the $V_{x}$ and $V_{y}$ components which could be performed independently for each plane is not unique. Norton (1989) showed that the irrotational part of a $2 \mathrm{D}$ vector field cannot be reconstructed from the vectorial ray transform. It can be reconstructed uniquely if $\nabla \cdot \boldsymbol{V}=0$ by solving a boundary value problem.

We extend the work of Norton (1989) to the case of a 3D vector field $\boldsymbol{V}(\boldsymbol{r})$ for which we have a Helmholz decomposition:

$$
\boldsymbol{V}=\nabla \times \boldsymbol{\Psi}+\nabla \cdot \Phi
$$

where $\boldsymbol{\Psi}$ and $\Phi$ are the vector and scalar potentials respectively, $\nabla \times \Psi$ and $\nabla \cdot \Phi$ are the solenoidal and irrotational parts of $\boldsymbol{V}$, respectively. The vectorial ray transform (3.3) can be rewritten in the form

$$
\begin{array}{r}
I_{\phi, \theta}\left(x_{p}, y_{p}\right)=\cos \phi \sin \theta \iiint_{\text {Corona }} V_{x}(x, y, z) \delta_{x p} \delta_{y p} d x d y d z+ \\
+\sin \phi \sin \theta \iiint_{\text {Corona }} V_{y}(x, y, z) \delta_{x p} \delta_{y p} d x d y d z+ \\
+\cos \theta \iiint_{\text {Corona }} V_{z}(x, y, z) \delta_{x p} \delta_{y p} d x d y d z,
\end{array}
$$

where the line integration is written as a volume integral with suitable Dirac-delta functions

$$
\begin{aligned}
& \delta_{x p}=\delta\left(x \sin \phi-y \cos \phi-x_{p}\right), \\
& \delta_{y p}=\delta\left(-x \cos \phi \cos \theta-y \sin \phi \cos \theta+z \sin \theta-y_{p}\right),
\end{aligned}
$$

are the Dirac delta-functions. Substituting (3.5) into the 2D Fourier transform of $I_{\phi, \theta}\left(x_{p}, y_{p}\right)$ with respect to the image position $\left(x_{p}, y_{p}\right)$,

$$
\tilde{I}_{\phi, \theta}\left(k_{1}, k_{2}\right)=\iint_{\text {Image of }(\phi, \theta)} I_{(\phi, \theta)}\left(x_{p}, y_{p}\right) e^{-i\left(k_{1} x_{p}+k_{2} y_{p}\right)} d x_{p} d y_{p}
$$

and changing the order of integration, one obtains

$$
\tilde{I}_{\phi, \theta}\left(k_{1}, k_{2}\right)=\cos \phi \sin \theta \tilde{V}_{x}(u, v, w)+\sin \phi \sin \theta \tilde{V}_{y}(u, v, w)+\cos \theta \tilde{V}_{z}(u, v, w),
$$

where $\tilde{V}_{x}, \tilde{V}_{y}$ and $\tilde{V}_{z}$ are the 3D-Fourier transforms of $V_{x}, V_{y}$ and $V_{z}$ respectively, with respect to the position in the corona $(x, y, z)$, and

$$
u=k_{1} \sin \phi-k_{2} \cos \phi \cos \theta, v=-k_{1} \cos \phi-k_{2} \sin \phi \cos \theta, w=k_{2} \sin \theta .
$$

If we have plane-parallel slices ( $\cos \theta=0$ ), then equation (3.8) represents the centralslice theorem for a 2D vector field. Taking into account (3.4) and (3.9), the 3D Fourier 
transform of the components of the vector $\boldsymbol{V}$ are

$$
\begin{aligned}
& \tilde{V}_{x}(u, v, w)=i v \tilde{\Psi}_{z}(u, v, w)-i w \tilde{\Psi}_{y}(u, v, w)+i u \tilde{\Phi}(u, v, w) \\
& \tilde{V}_{y}(u, v, w)=i w \tilde{\Psi}_{x}(u, v, w)-i u \tilde{\Psi}_{z}(u, v, w)+i v \tilde{\Phi}(u, v, w) \\
& \tilde{V}_{z}(u, v, w)=i u \tilde{\Psi}_{y}(u, v, w)-i v \tilde{\Psi}_{x}(u, v, w)+i w \tilde{\Phi}(u, v, w)
\end{aligned}
$$

Placing (3.10) into (3.8), one has

$$
\begin{aligned}
& \tilde{I}_{\phi, \theta}\left(k_{1}, k_{2}\right)=i\left(k_{1} \cos \phi \cos \theta+k_{2} \sin \phi\right) \tilde{\Psi}_{x}+ \\
& +i\left(k_{1} \sin \phi \cos \theta-k_{2} \cos \phi\right) \tilde{\Psi}_{y}-i k_{1} \sin \theta \tilde{\Psi}_{z},
\end{aligned}
$$

which is independent on the source $\tilde{\Phi}$ of the vector field. This means that even with measurements from all possible directions in 3D space, it is impossible to reconstruct the irrotational component of the vector field.

\subsection{Matrix formulation of the vector tomography prob- lem}

Like in the scalar field tomographic problem, vector tomography for the $I^{\|}$-type data (3.2) can be represented in matrix equation form as well:

$$
\mathbf{A} \cdot \mathbf{X}=\mathbf{Y}
$$

Here, the components $x_{3 j-k}(k=2,1,0)$ of the column-vector $\mathbf{X}$ contain components of the vector $\boldsymbol{B}$ in the $j$-cell, and $y_{i}$ is the data value for the $i$-th ray, where the index $i=1, \ldots, m$ counts both view angles, $\phi$ and $\theta$, and pixel position $\left(x_{p}, y_{p}\right)$. The matrix elements $a_{i, 3 j-k}$ represents the projection of volume element $j$ onto the LOS, related to the pixel $i$, multiplied with the components of a unit vector co-directed with the LOS; i.e. $a_{i, 3 j-k}$ are the components of vector $\Delta \boldsymbol{l}$. Here we do not take into the account the electron density dependence of the measured data. The way how to include this dependence into the matrix $\mathbf{A}$ will be described in section 6.3.

\subsection{Special regularization for coronal vector tomography}

Taking into account that magnetic field is free of divergence $(\nabla \cdot \boldsymbol{B}=0)$, it is possible to introduce a regularization term into the minimizing function of the form:

$$
F=F_{\text {tomo }}+\mu F_{\text {reg }}=\sum_{\text {all LoS }}\left(I^{\text {sim }}-I^{\text {obs }}\right)^{2}+\mu \sum_{\text {Corona }}|\nabla \cdot \boldsymbol{B}(\boldsymbol{r})|^{2} .
$$

By applying finite difference approach to $\nabla \cdot \boldsymbol{B}$, one gets a matrix form for the regularization term:

$$
F_{\text {reg }}=\left|\mathbf{A}_{\text {reg }} \cdot \mathbf{X}-\mathbf{Y}_{\mathrm{b}}\right|^{2},
$$

where the column vector $\mathbf{X}$ is the same as in (3.12). The number of rows in $\mathbf{A}_{\text {reg }}$ is equal to the number of cells involved in the calculations (equal to the number of elements in $\mathbf{X}$ 
divided by three). Matrix $\mathbf{Y}_{\mathrm{b}}$ contains boundary values defined from the measurements of the magnetic field at the photospheric level.

This regularization is different from the one presented for the scalar field tomography, where the regularization term represents the roughness of the solution and its minimization ensures a certain degree of smoothness of the solution. The regularization $\nabla \cdot \boldsymbol{B}=0$ has large null space and acts only on the irrotational part of the field. The null space is therefore composed of all divergencefree fields compatible with the measured boundary condition $\mathbf{Y}_{\mathrm{b}}$. It is well known (Bellan 2000) that the field with the smallest energy, i.e. $|\mathbf{X}|^{2}=\sum_{\text {corona }}|\boldsymbol{B}|^{2}$, within this null space is the potential field that fits the boundary conditions. Many solution algorithms for (3.13) implicitly minimize the solution norm $|\mathbf{X}|^{2}$. Hence, such an algorithm will yield the potential field model of the corona if no tomography data is present and only the second term in (3.13) is minimized. Any tomography data will yield possible non-potential extensions of the coronal magnetic field. The numerical implementation of the term will be further described in the section 6.2. 



\section{The possible effects used for deriving the magnetic field}

\subsection{Zeeman-effect}

The Zeeman-effect was discovered by Zeeman (1897), and consists in the splitting of individual spectral lines of an emission spectrum into three or more polarized components in presence of a magnetic field. When spectral lines split into the three components, $\sigma_{b}$, $\sigma_{r}$ and $\pi$, the effect is known as normal Zeeman effect. The case when spectral lines split into more than three components is known as anomalous Zeeman effect (Figure 4.1).

In the presence of a magnetic field each level with the magnetic quantum number $M_{J}$ gets additional energy

$$
E_{B}=g M_{J} \hbar \frac{e B}{2 m_{e}}=g \hbar \omega_{L},
$$

where $e$ is the elementary charge, $B$ the magnetic field strength, $m_{e}$ the mass of the electron, $\hbar$ the Planck constant, $\omega_{L}$ is the Larmour frequency, and $g$ is the Lande factor depending on the quantum numbers $L, S, J$ and $M_{J}: L$ is for orbital angular momentum of the electrons, $S$ is their spin quantum number, $J$ is the associated total angular momentum quantum number, and $M_{J}$ is the quantum number for the component of total angular momentum along the direction of the magnetic field (magnetic quantum number). The Landé factor is

$$
g=\frac{3}{2}+\frac{S(S+1)-L(L+1)}{2 J(J+1)} .
$$

Each level with total angular momentum $J$ splits into $(2 J+1)$ sublevels. As a result, the frequencies related to the transitions between the lower level with $J_{l}$ and upper level with $J_{u}$ are defined by

$$
\nu_{J_{l} M_{l} \leftrightarrow J_{u} M_{u}}=\nu_{0}+\frac{e B}{2 m_{e}} \cdot\left(g_{u} M_{u}-g_{l} M_{l}\right)
$$

where $\nu_{0}$ is the frequency of the line in the absence of magnetic field, $g_{u}$ and $g_{l}$ are the Landé factors for the upper and lower levels respectively, and $M_{u}$ and $M_{l}$ are the magnetic quantum numbers for these levels.

The selection rules for allowed electric dipole (E1) transitions are:

$$
\begin{array}{r}
\Delta J=0, \pm 1, J_{u}=0 \rightarrow J_{l}=0 \text { is forbidden } \\
\Delta L=0, \pm 1, \Delta S=0, \\
\Delta M=0, \pm 1 .
\end{array}
$$



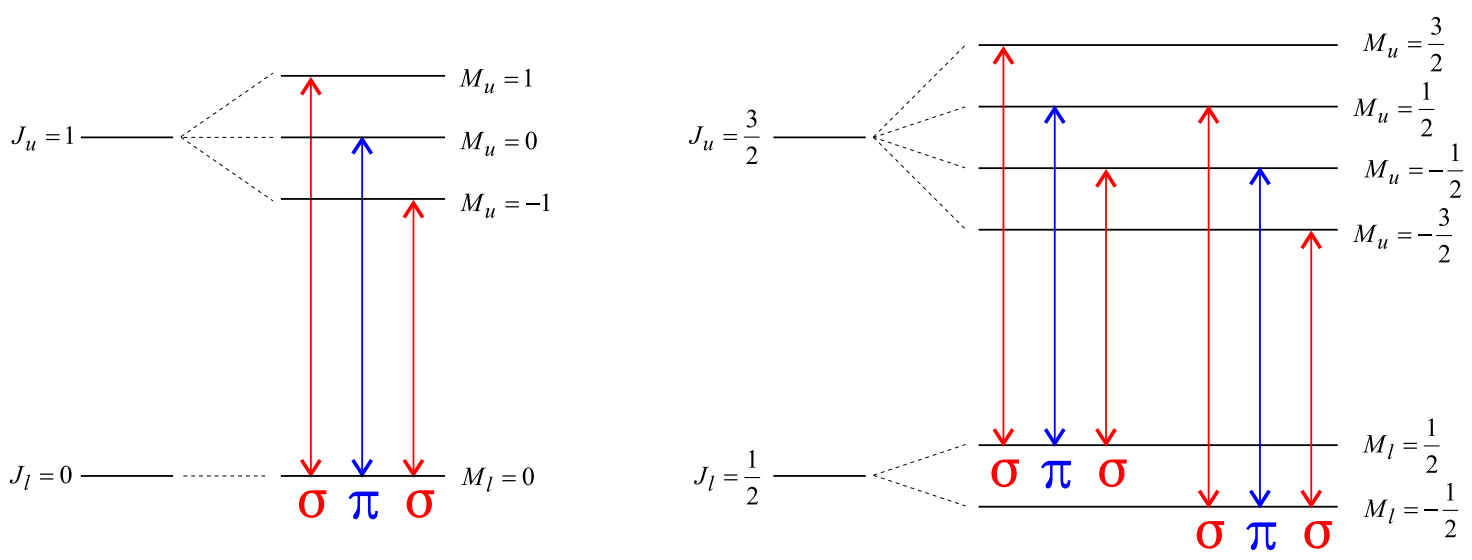

Figure 4.1: Zeeman transitions.

The selection rule for magnetic dipole (M1) transitions is $\Delta M=0, \pm 1$. The lines $5303 \AA$ and $10747 \AA$ of the Fe XIV and Fe XIII ions, respectively, are forbidden for the electric dipole (E1) transitions but allowed for the magnetic dipole (M1) transitions. The $\pi$-components correspond to the transitions with $\Delta M=0$, and $\sigma$-components - with $\Delta M= \pm 1$ (Fig. 4.1). If the LOS is parallel to the direction of the magnetic field, clockwise polarization at frequency $\nu_{\sigma_{b}}$ will be observed and counterclockwise at the frequency $\nu_{\sigma_{r}}$ (longitudinal Zeeman-effect), where

$$
\begin{array}{r}
\nu_{\sigma_{b}}=\nu_{0}-\Delta \nu_{Z}, \\
\nu_{\sigma_{b}}=\nu_{0}-\Delta \nu_{Z}, \\
\Delta \nu_{Z}=\frac{e B}{2 m_{e}} \cdot\left|g_{u} M_{u}-g_{l} M_{l}\right| .
\end{array}
$$

If we observe along the direction perpendicular to the magnetic field vector, we see a linear polarization parallel to the vector $\boldsymbol{B}$ at frequency $\nu=\nu_{0}$ and a linear polarization perpendicular to the $\boldsymbol{B}$ at $\nu=\nu_{0} \pm \Delta \nu_{Z}$ (transversal Zeeman-effect). The intensity of the circularly polarized signal corresponding to the longitudinal Zeeman-effect is much higher than intensity of the linear polarized signal for the transversal one.

\subsection{Hanle-effect}

The Hanle-effect was discovered by Hanle in 1923 (Hanle 1924). Here we briefly describe the key points of this effect. A more detailed description can be found in Mitchel \& Zemansky (1934), and Landi \& Landolfi (2004). Let us consider scattering of unpolarized light of an atom with $J_{l}=0$ and $J_{u}=1$ without magnetic field (Fig. 4.2a). If no magnetic field present, the atom can be described by three independent linear oscillators. The radiation scattered along the direction of incident light is unpolarized. But if we observe from a direction perpendicular to the incident light, we find linear polarization.

Let us introduce now a magnetic field parallel to the $x$-axis (Fig. 4.2b,c). Then the atom must be described by a linear oscillator parallel to the magnetic field and two counter-rotating circular oscillators in the $y z$-plane oscillating at frequencies $\omega_{0}-\omega_{L}$ and 


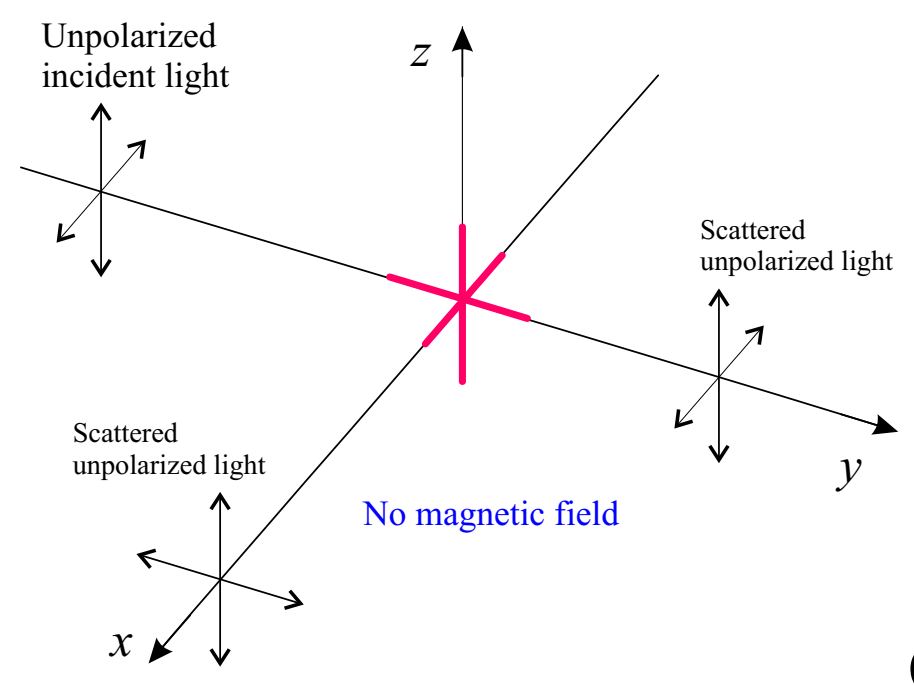

(a)
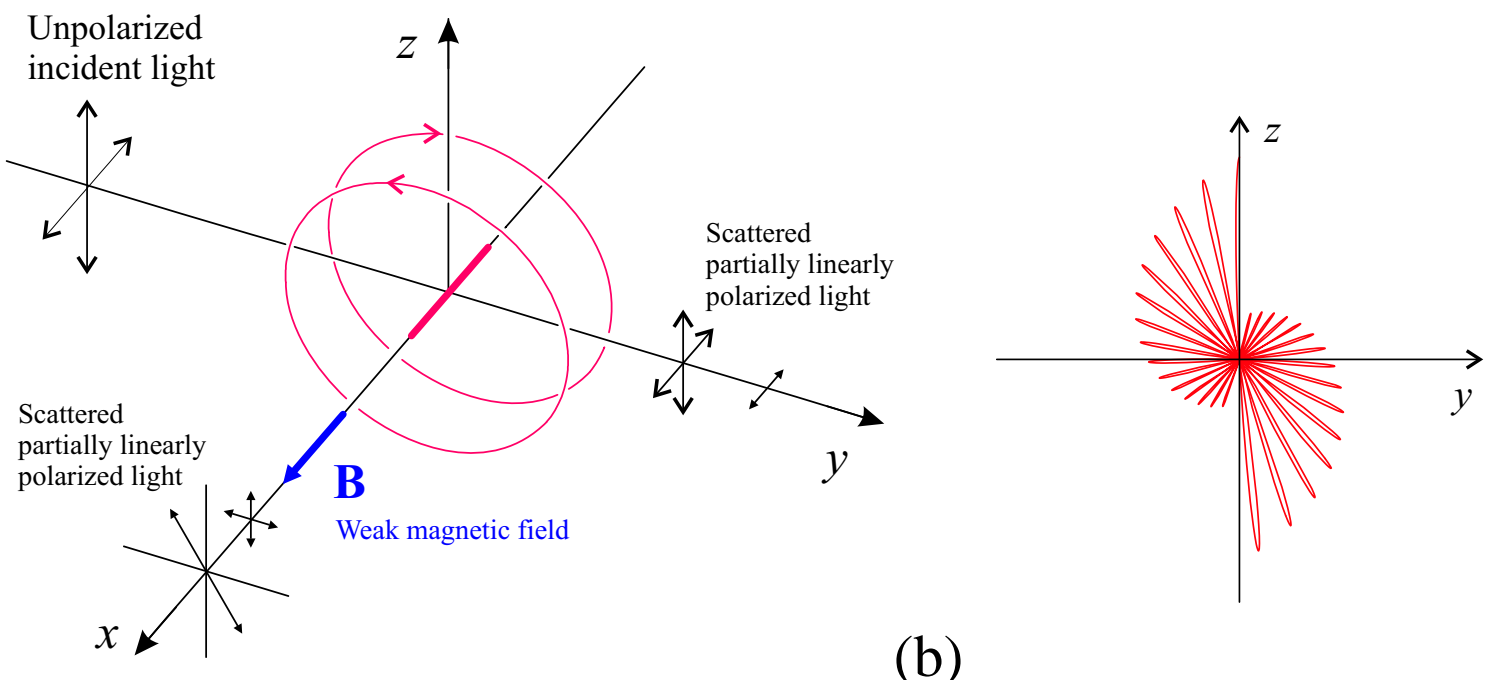

(b)
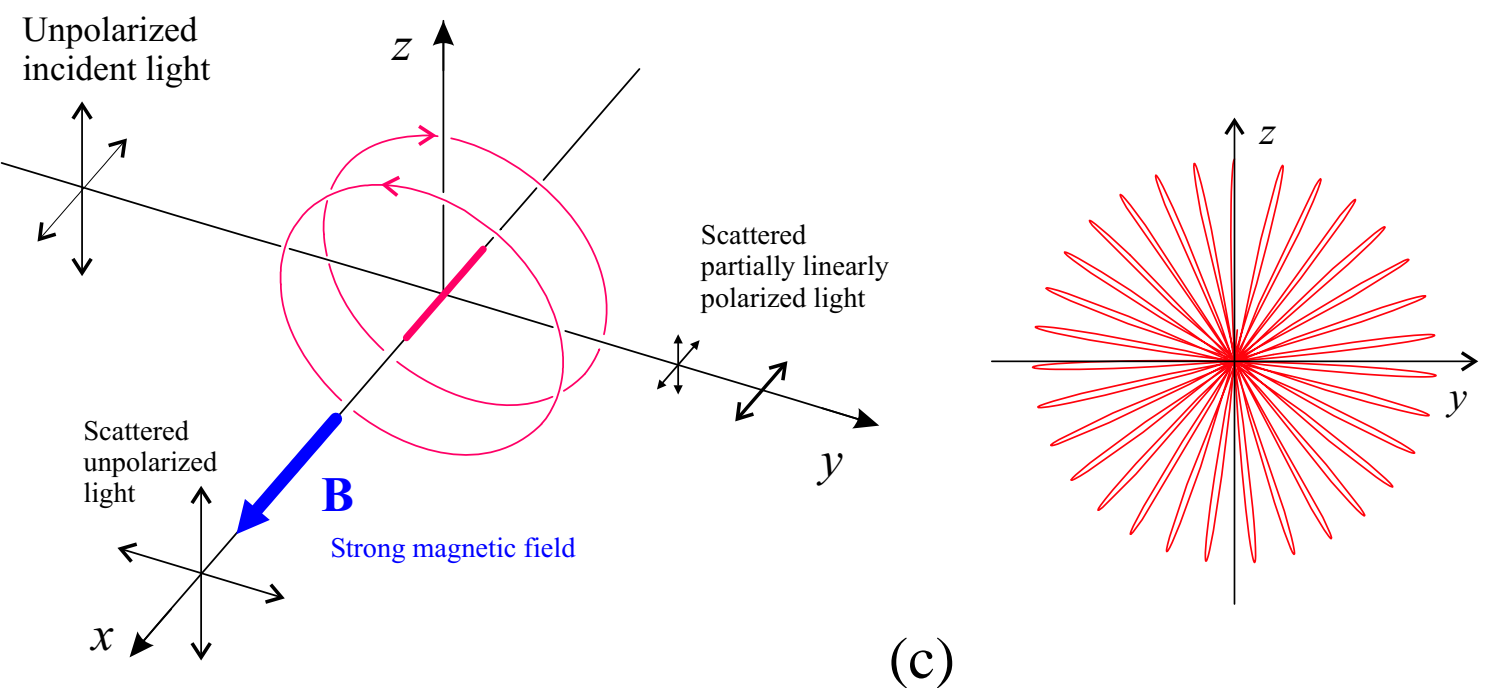

(c)

Figure 4.2: Polarization of forward and perpendicular scattered radiation in the absence of magnetic field (a) and in the presence of the field parallel to the perpendicular LOS direction $(b, c)$. 
$\omega_{0}+\omega_{L}$, where $\omega_{L}$ is the Larmor frequency. The resulting trajectory of the electron in the $y z$-plane is (Trujillo Bueno 2000)

$$
\left\{\begin{array}{l}
y(t)=A \cdot e^{-t / 2 \tau} \cos \left(\omega_{L} t\right) \cos \left(\omega_{0} t\right) \\
z(t)=A \cdot e^{-t / 2 \tau} \sin \left(\omega_{L} t\right) \cos \left(\omega_{0} t\right)
\end{array}\right.
$$

where $\tau$ is a life time.

When the magnetic field is strong $\left(\omega_{L} \tau \gg 1\right)$, the oscillation axis rotates several times (Fig. 4.2c). The trajectory of the electron in $y z$-plane is a "daffodil", and the scattered light into the perpendicular direction is totally unpolarized. For the forward scattering case we have a maximum amount of linear polarization.

In case of the weak magnetic field $\left(\omega_{L} \tau \approx 1\right)$, the oscillation axis rotates only for some angle $\alpha$ (figure 4.2b). The electron now describes a "rosette" in the $y z$-plane, with result that the radiation scattered into the $x$-direction will be partially polarized. In comparison with non-magnetic case, we have a decreased degree of polarization and rotation of the polarization plane.

\subsection{Faraday-effect}

Linearly polarized radiation can be described as the superposition of two circularly polarized components with opposite rotation. The total electric field vector in case of a propagation along the $z$-direction is splitted in a sum of two electric vectors, $\boldsymbol{E}_{\boldsymbol{R}}$ and $\boldsymbol{E}_{\boldsymbol{L}}$, corresponding to the right- and left-hand circularly polarized field components,

$$
\boldsymbol{E}=\boldsymbol{E}_{\boldsymbol{R}}+\boldsymbol{E}_{\boldsymbol{L}}=\left(\hat{\boldsymbol{e}}_{\boldsymbol{x}}+i \hat{\boldsymbol{e}}_{\boldsymbol{y}}\right) E_{0 R} \mathrm{e}^{i\left(k_{R} z-\omega t\right)}+\left(\hat{\boldsymbol{e}}_{\boldsymbol{x}}-i \hat{\boldsymbol{e}}_{\boldsymbol{y}}\right) E_{0 L} \mathrm{e}^{i\left(k_{L} z-\omega t\right)},
$$

where $E_{0 L}$ and $E_{0 R}$ are the amplitudes for the left- and right-hand circularly polarized components, respectively, $k_{L}$ and $k_{R}$ are their respective wave vectors in the $z$-direction, $\omega$ is the cyclic frequency, and $t$ is the time, $\hat{e}_{\boldsymbol{x}}$ and $\hat{\boldsymbol{e}}_{\boldsymbol{y}}$ are the unit vectors defining the $x$ and $y$-directions, respectively.

The polarization angle $\phi$ is defined through the ratio of the $x$ - and $y$-components of the electric field vector,

$$
\cot \phi=\frac{E_{x}}{E_{y}}=\frac{\frac{E_{0 L}}{E_{0 R}} \cos \left(k_{L} z+\omega t\right)+\cos \left(k_{R} z-\omega t\right)}{\frac{E_{0 L}}{E_{0 R}} \sin \left(k_{L} z+\omega t\right)+\sin \left(k_{R} z-\omega t\right)},
$$

and for the circular polarization, when $E_{0 R}=E_{0 R}$, we have

$$
\cot \phi=\cot \left(\frac{k_{L}-k_{R}}{2} z\right)
$$

The values of the propagation vectors $k_{L}$ and $k_{R}$ may differ in a magnetized plasma. We consider radio or light waves for which the frequency $\omega$ is much larger than plasma frequency

$$
\omega_{p}=\frac{n_{e} e^{2}}{m_{e} \epsilon_{0}}
$$


where $n_{e}$ is the electron density, $m_{e}$ the electron mass, $e$ the elementary charge and $\epsilon$ the electric constant. For our purpose the wave numbers as derived in magneto-ionic theory (Stix 1962) therefore are

$$
k_{L}=\frac{\omega}{c} \sqrt{1-\frac{\omega_{p}^{2}}{\omega\left(\omega+\omega_{e}\right)}}, \quad k_{R}=\frac{\omega}{c} \sqrt{1-\frac{\omega_{p}^{2}}{\omega\left(\omega-\omega_{e}\right)}} .
$$

Here, $c$ is the speed of light,

$$
\omega_{e}=\frac{e B_{z}}{m_{e}}
$$

is the electron gyrofrequency, and $B_{z}$ is the projection of the magnetic field vector on the propagation direction $z$. For the radio waves and typical coronal magnetic field strength $\omega \gg \omega_{e}$. So, the total rotation of the polarization plane of the linearly polarized electromagnetic wave after passing the corona is

$$
\phi=\frac{e^{3}}{2 \pi m_{e}^{2} c^{2}(\omega / 2 \pi)^{2}} \int n_{e} B_{z} d z .
$$

Since the rotation angle is inversely proportional to the square of radiation frequency, the Faraday-effect is more effective for radio waves. The Faraday rotation can be used for the determination of the magnetic field in the corona by polarization measurements before and during the occultation by the Sun of a distant linearly polarized radio source. For example, Stelzried et al. (1970) observed this effect between $4-12 R_{\odot}$ during the Pioneer 6 passage behind the Sun. Golnev et al. (1969) found a magnetic field strength of $10^{-2} \mathrm{G}$ for the corona at a distance of $5 R_{\odot}$ during the occultation of the Crab Nebula. 



\section{The line formation of magnetically sensitive lines}

The processes of the formation of polarized emission of coronal lines in presence of a magnetic field is briefly described in this chapter. The emission lines are formed due to atomic transitions from an upper excited state to lower one. The excited state of an atom in the corona is mainly caused by thermal collisions or by radiation incident from the photosphere. The polarization of the emitted radiation is due to the anisotropy of the incident pumping radiation and the anisotropy induced by the magnetic field. The magnetograph formula expresses the dependence of the Stokes- $I$ and $-V$ spectra from the LOS projection of the magnetic field vector. This formula is the background for the tomographic reconstruction based on the longitudinal Zeeman-effect data. The expressions for calculation of the Stokes- $I,-Q$ and $-U$ vectors, integrated over the line profiles for the infrared $(10747 \AA)$ and green $(5303 \AA)$ lines, are presented in the sections 5.10 and 5.11. These expressions are directly involved in the reconstruction procedure using the Hanle-effect data described in the next chapter.

\subsection{Stokes vector}

The electric field vector of the monochromatic electromagnetic wave propagating in the $z$-direction is expressed by

$$
\begin{aligned}
& E_{x}(t)=E_{x} \cos \left(\omega t-\phi_{x}\right) \\
& E_{y}(t)=E_{y} \cos \left(\omega t-\phi_{y}\right)
\end{aligned}
$$

where $E_{x}$ and $E_{y}$ are the amplitudes, $\phi_{x}$ and $\phi_{y}$ are phase shifts, $\omega$ is the cyclic frequency and $t$ the time. Instead of these four parameters describing the wave $\left(E_{x}, E_{y}, \phi_{x}, \phi_{y}\right)$, G.Stokes introduced another set:

$$
\begin{array}{r}
I_{\omega}=E_{x}^{2}+E_{y}^{2}, \\
Q_{\omega}=E_{x}^{2}-E_{y}^{2}, \\
U_{\omega}=2 E_{x} E_{y} \cos \left(\phi_{x}-\phi_{y}\right), \\
V_{\omega}=2 E_{x} E_{y} \sin \left(\phi_{x}-\phi_{y}\right) .
\end{array}
$$

These Stokes parameters satisfy the relationship:

$$
I_{\omega}^{2}=Q_{\omega}^{2}+U_{\omega}^{2}+V_{\omega}^{2}
$$


The Stokes parameters are chosen in the way that $I_{\omega}$ describes the total intensity of polarized monochromatic light, $Q_{\omega}$ is the intensity of linearly polarized radiation along $x$-axis minus intensity of linearly polarized radiation along $y$-axis, $U_{\omega}$ the intensity of linearly polarized radiation along the unit vector $\left(\hat{\boldsymbol{e}}_{x}+\hat{\boldsymbol{e}}_{y}\right) / \sqrt{2}$ minus intensity of linearly polarized radiation along $\left(-\hat{\boldsymbol{e}}_{x}+\hat{\boldsymbol{e}}_{y}\right) / \sqrt{2}$, and $V_{\omega}$ the intensity of right circularly polarized radiation minus the intensity of left circularly polarized radiation.

But the monochromatic approximation of the wave is far from reality. A more realistic description of electromagnetic radiation is the superposition of many wave packets having a limited extension in space and time (quasi-monochromatic radiation). Therefore the Stokes-parameters now is expressed by averaging over the statistical distribution of the wave packets:

$$
\begin{array}{r}
I=\left\langle E_{x}^{2}+E_{y}^{2}\right\rangle, \\
Q=\left\langle E_{x}^{2}-E_{y}^{2}\right\rangle, \\
U=\left\langle 2 E_{x} E_{y} \cos \left(\phi_{x}-\phi_{y}\right)\right\rangle, \\
V=\left\langle 2 E_{x} E_{y} \sin \left(\phi_{x}-\phi_{y}\right)\right\rangle .
\end{array}
$$

Now $I^{2} \geq Q^{2}+U^{2}+V^{2}$, and total intensity, $I$, is a sum of the intensities of the polarized component and unpolarized one:

$$
I=\sqrt{Q^{2}+U^{2}+V^{2}}+I_{\text {unpolarized }} .
$$

The relation

$$
p=\frac{\sqrt{Q^{2}+U^{2}+V^{2}}}{I}
$$

defines the degree of polarization.

\subsection{Radiative Transfer for the Polarized Radiation}

Under the assumption that the corona is optically thin, the radiative transfer equation (RTE) for polarized radiation takes form

$$
\frac{d \mathbf{S}}{d l}=\mathbf{J}
$$

where $\mathbf{S}=(I, Q, U, V)^{\mathrm{T}}$ is the Stokes vector (sign "T" here notes matrix transpose operation), $\mathbf{J}=\left(\varepsilon_{I}, \varepsilon_{Q}, \varepsilon_{U}, \varepsilon_{V}\right)^{\mathrm{T}}$ is the emission vector, and $l$ is the coordinate along the LOS. The emission coefficients in the emission vector for the magnetic dipole transition for polarized radiation can be written in a similar way like for the unpolarized case, but we have to take into the account the dependence of the Stokes components on the orientation of the magnetic dipole with respect to the chosen reference system (Fig. 5.1) (Landi \& Landolfi 2004):

$$
\begin{gathered}
\varepsilon_{I}=\operatorname{Re}\left(f_{++}+f_{--}\right), \\
\varepsilon_{Q}=-\operatorname{Re}\left(f_{+-}+f_{-+}\right), \\
\varepsilon_{U}=\operatorname{Im}\left(f_{+-}-f_{-+}\right), \\
\varepsilon_{V}=\operatorname{Re}\left(f_{++}-f_{--}\right),
\end{gathered}
$$


where for the case of magnetic dipole transitions

$$
\begin{array}{r}
f_{\alpha \beta}=\frac{2 h \nu^{3}}{c^{2}} \frac{4 \pi^{3} \nu}{c h} N \times \\
\times \sum_{m m^{\prime} n}\left[\boldsymbol{\mu}_{m n} \cdot \boldsymbol{\Omega} \times \hat{\boldsymbol{e}}_{\alpha}(\boldsymbol{\Omega})\right]\left[\boldsymbol{\mu}_{n m^{\prime}} \cdot \boldsymbol{\Omega} \times \hat{\boldsymbol{e}}_{\beta}(\boldsymbol{\Omega})^{*}\right] \rho_{m^{\prime} m} \Phi\left(\nu_{m n}-\nu\right) .
\end{array}
$$

Here, $\boldsymbol{\mu}_{m n}$ is magnetic dipole moment for the transition $m \rightarrow n, \rho_{m^{\prime} m}$ is a density matrix element that should be found by solving statistical-equilibrium equations, and $N$ is the density of the radiating ions. Here the reference system defined by the unit vectors $\left(\hat{\boldsymbol{e}}_{\mathrm{a}}, \hat{\boldsymbol{e}}_{\mathrm{b}}\right)$ was replaced by the circulating reference system defining by the unit vectors

$$
\begin{gathered}
\hat{\boldsymbol{e}}_{-1}(\boldsymbol{\Omega})=\frac{1}{\sqrt{2}}\left[\hat{\boldsymbol{e}}_{\mathrm{a}}(\boldsymbol{\Omega})+\mathrm{i} \hat{\boldsymbol{e}}_{\mathrm{b}}(\boldsymbol{\Omega})\right], \\
\hat{\boldsymbol{e}}_{+1}(\boldsymbol{\Omega})=\frac{1}{\sqrt{2}}\left[-\hat{\boldsymbol{e}}_{\mathrm{a}}(\boldsymbol{\Omega})+\mathrm{i} \hat{\boldsymbol{e}}_{\mathrm{b}}(\boldsymbol{\Omega})\right]
\end{gathered}
$$

The function $\Phi$ is a generalized complex line profile expressed in units of Doppler width,

$$
\Delta \lambda_{D}=\lambda_{0} \frac{v_{T}}{c}, \quad \Delta \nu_{D}=\nu_{0} \frac{v_{T}}{c}
$$

where $v_{T}$ is the thermal velocity, $c$ the speed of light, and $\lambda_{0}$ and $\nu_{0}$ define the position of the gravity center of the spectrum in wavelength or frequency (when a macroscopic velocity is not taken into account). The profile of the function $\Phi$ can be written as:

$$
\Phi=\frac{1}{\sqrt{\pi} \Delta \nu_{D}} \cdot H\left(\frac{\nu_{m n}-\nu}{\Delta \nu_{D}}-v_{\mathrm{A}}, a\right)+\frac{\mathrm{i}}{\sqrt{\pi} \Delta \nu_{D}} \cdot L\left(\frac{\nu_{m n}-\nu}{\Delta \nu_{D}}-v_{\mathrm{A}}, a\right)
$$

where

$$
a=\frac{\Gamma}{\Delta \nu_{D}}=\frac{\Gamma \lambda_{0}^{2}}{c \Delta \lambda_{D}}
$$

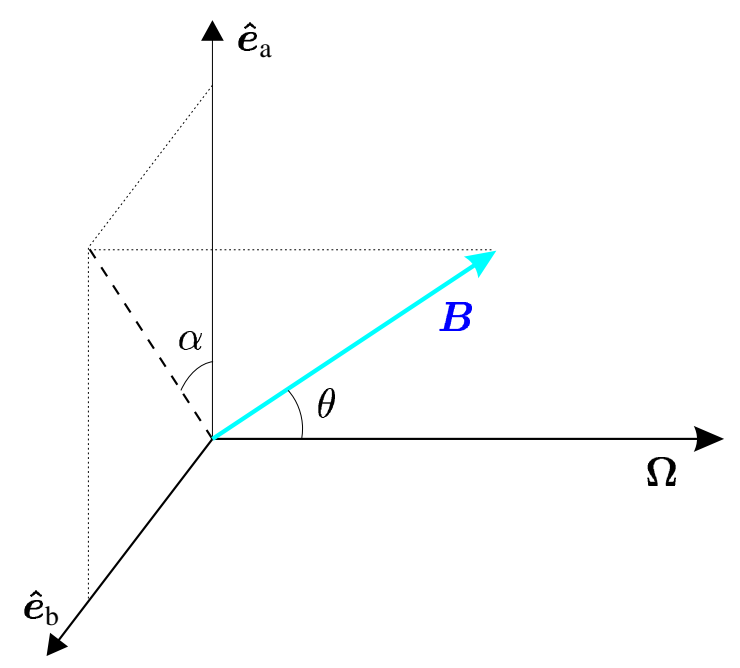

Figure 5.1: The unit vector of reference direction, $\hat{\boldsymbol{e}}_{\mathrm{a}}$, and associated unit vector $\hat{\boldsymbol{e}}_{\mathrm{b}}$ so that $\hat{\boldsymbol{e}}_{\mathrm{a}} \times \hat{\boldsymbol{e}}_{\mathrm{b}} \uparrow \uparrow \boldsymbol{\Omega}$, where $\boldsymbol{\Omega}$ is propagation direction (LOS direction). 
Here $\Gamma=\left(\gamma_{m}+\gamma_{n}\right) / 4 \pi$ is the damping parameter, and $\gamma_{m}$ and $\gamma_{n}$ are probabilities per unit time that the atom leaves the level $m$ or $n$.

The functions

$$
\begin{aligned}
H(v, a) & =\frac{a}{\pi} \int_{-\infty}^{+\infty} \mathrm{e}^{-y^{2}} \frac{1}{(v-y)^{2}+a^{2}} d y \\
L(v, a) & =\frac{1}{\pi} \int_{-\infty}^{+\infty} \mathrm{e}^{-y^{2}} \frac{v-y}{(v-y)^{2}+a^{2}} d y
\end{aligned}
$$

are the Voigt-function and the associated dispersion profile, respectively, obtained by the convolution of a Maxwell distribution function (thermal broadening) with a Lorentzian profile, and $v_{\mathrm{A}}$ is the Doppler shift induced by LOS component of the macroscopic velocity, $v_{\text {LOS }}$ :

$$
v_{\mathrm{A}}=\frac{v_{\mathrm{LOS}}}{v_{T}}=\frac{\lambda_{0} v_{\mathrm{LOS}}}{c \Delta \lambda_{D}}=\frac{\nu_{0} v_{\mathrm{LOS}}}{c \Delta \nu_{D}}
$$

where under the assumption of a Maxwellian velocity distribution and single-atomic gas $v_{T}=\sqrt{3 k T / m_{0}}\left(k\right.$ is the Boltzmann constant, and $m_{0}$ is the mass of the atom).

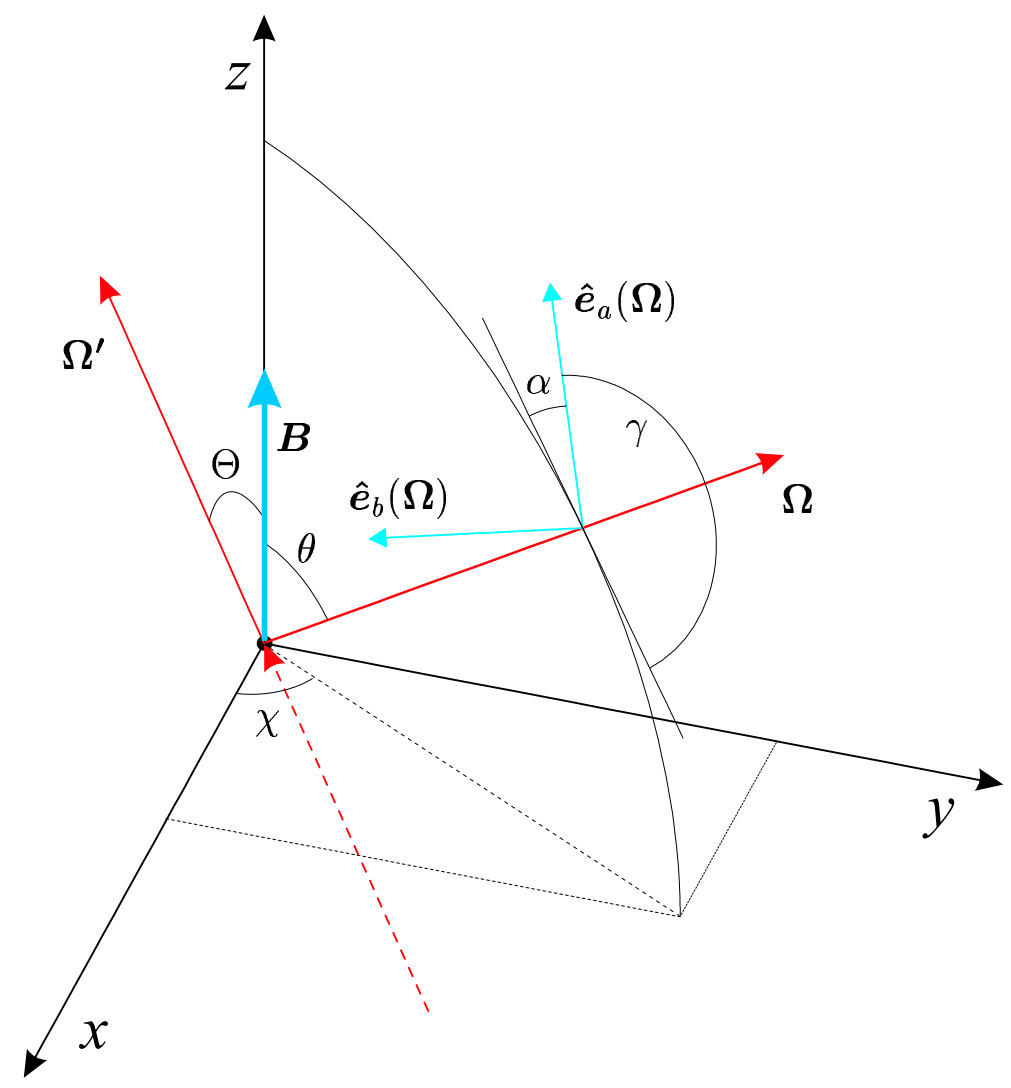

Figure 5.2: The geometry of the scattering process. The vectors $\boldsymbol{\Omega}^{\prime}$ and $\boldsymbol{\Omega}$ define the directions of the incident and scattered radiation, respectively. The unit vectors of polarization reference $\hat{\boldsymbol{e}}_{a}$ and $\hat{\boldsymbol{e}}_{b}$ are chosen in the way that $\hat{\boldsymbol{e}}_{a} \perp \hat{\boldsymbol{e}}_{b}, \hat{\boldsymbol{e}}_{\mathrm{a}} \times \hat{\boldsymbol{e}}_{\mathrm{b}} \uparrow \boldsymbol{\Omega}$, and $\hat{\boldsymbol{e}}_{a}$ lies in the plane defined by the vectors $\Omega$ and $\boldsymbol{\Omega}^{\prime}$. 
Let us consider an atomic system without hyperfine structure, and with energy levels characterized by the quantum numbers $\alpha$ representing the set of quantum numbers (electronic configuration, total orbital angular momentum, $L$, total electronic spin, $S$ ), $J$ representing the total angular momentum, and magnetic quantum number $M$.

Substituting these quantum numbers in (5.16) leads to the subsequent replacements:

$$
n \rightarrow \alpha_{l} J_{l} M_{l}, \quad m \rightarrow \alpha_{u} J_{u} M_{u}, \quad m^{\prime} \rightarrow \alpha_{u} J_{u} M_{u}^{\prime}
$$

where indexes "l" and " $u$ " specify lower and upper levels between which electron transitions occur. Applying the Wigner-Eckart theorem to the spherical components of the dipole operator, and introducing the Einstein coefficient, $A$, for the magnetic dipole spontaneous transition $\alpha_{u} J_{u} \rightarrow \alpha_{l} J_{l}$, we get the spontaneous emission coefficients, $\left(\varepsilon_{I}, \varepsilon_{Q}, \varepsilon_{U}, \varepsilon_{V}\right)=$ $\left(\varepsilon_{0}, \varepsilon_{1}, \varepsilon_{2}, \varepsilon_{3}\right)$, in the form:

$$
\begin{array}{r}
\varepsilon_{i}(\nu, \Omega)=\frac{h \nu}{4 \pi} N \sum_{\alpha_{l} J_{l}} \sum_{\alpha_{u} J_{u}}\left(2 J_{u}+1\right) A_{\alpha_{u} J_{u} \rightarrow \alpha_{l} J_{l}} \times \\
\times \sum_{M_{u} M_{u}^{\prime} M_{l} q q^{\prime}} 3\left(\begin{array}{ccc}
J_{u} & J_{l} & 1 \\
-M_{u} & M_{l} & -q
\end{array}\right)\left(\begin{array}{ccc}
J_{u} & J_{l} & 1 \\
-M_{u}^{\prime} & M_{l} & -q^{\prime}
\end{array}\right) \times \\
\times \operatorname{Re}\left[\mathcal{T}_{q q^{\prime}}(i, \Omega) \rho_{\alpha_{u} J_{u}}\left(M_{u}^{\prime}, M_{u}\right) \Phi\left(\nu_{\alpha_{u} J_{u} M_{u}, \alpha_{l} J_{l} M_{l}}-\nu\right)\right]
\end{array}
$$

Here, $\mathcal{T}_{q q^{\prime}}(i, \Omega)$ with $\left(q, q^{\prime}=0, \pm 1\right)$ is the reducible spherical tensor (Table A.1) specifying the geometry of the observer (Fig. 5.2), and $\Phi$ is the complex line profile centered at $\nu_{0}=\nu_{\alpha_{u} J_{u} M_{u}, \alpha_{l} J_{l} M_{l}}$. It can be decomposed as follows:

$$
\Phi\left(\nu_{a b}-\nu\right)=\phi\left(\nu_{a b}-\nu\right)+\mathrm{i} \psi\left(\nu_{a b}-\nu\right),
$$

where $\phi$ is the Voigt profile and $\psi$ is the associated Faraday-Voigt dispersion function. The expressions in the matrix form in (5.25) are Wigner $3 j$-symbols.

\subsection{Statistical-equilibrium equation}

The density matrix element $\rho_{\alpha J}\left(M, M^{\prime}\right)$ can be found by solving the statistical-equilibrium equation, which involves any possible transition between the level of interest, $(\alpha, J)$, and upper, $\left(\alpha_{u}, J_{u}\right)$, and lower, $\left(\alpha_{l}, J_{l}\right)$, levels:

$$
\begin{array}{r}
\frac{d}{d t} \rho_{\alpha J}\left(M, M^{\prime}\right)=-2 \pi \mathrm{i} \nu_{L} g_{\alpha J}\left(M-M^{\prime}\right) \rho_{\alpha J}\left(M, M^{\prime}\right)+ \\
+\sum_{\alpha_{l} J_{l}} \sum_{M_{l} M_{l}^{\prime}} \rho_{\alpha_{l} J_{l}}\left(M_{l}, M_{l}^{\prime}\right) T_{\mathrm{A}}\left(\alpha J M M^{\prime}, \alpha_{l} J_{l} M_{l} M_{l}^{\prime}\right)+ \\
-\sum_{\alpha_{u} J_{u}} \sum_{M_{u} M_{u}^{\prime}} \rho_{\alpha_{u} J_{u}}\left(M_{u}, M_{u}^{\prime}\right)\left(T_{\mathrm{E}}\left(\alpha J M^{\prime}, \alpha_{u} J_{u} M_{u} M_{u}^{\prime}\right)+T_{\mathrm{S}}\left(\alpha J M M^{\prime}, \alpha_{u} J_{u} M_{u} M_{u}^{\prime}\right)\right)- \\
-\sum_{M^{\prime \prime}}\left[\rho_{\alpha J}\left(M, M^{\prime \prime}\right)\left(R_{\mathrm{A}}\left(\alpha J M^{\prime} M^{\prime \prime}\right)+R_{\mathrm{E}}\left(\alpha J M^{\prime \prime} M^{\prime}\right)+R_{\mathrm{S}}\left(\alpha J M^{\prime \prime} M^{\prime}\right)\right)+\right. \\
\left.+\rho_{\alpha J}\left(M^{\prime \prime}, M^{\prime}\right)\left(R_{\mathrm{A}}\left(\alpha J M^{\prime \prime} M\right)+R_{\mathrm{E}}\left(\alpha J M M^{\prime \prime}\right)+R_{\mathrm{S}}\left(\alpha J M M^{\prime \prime}\right)\right)\right],(5
\end{array}
$$




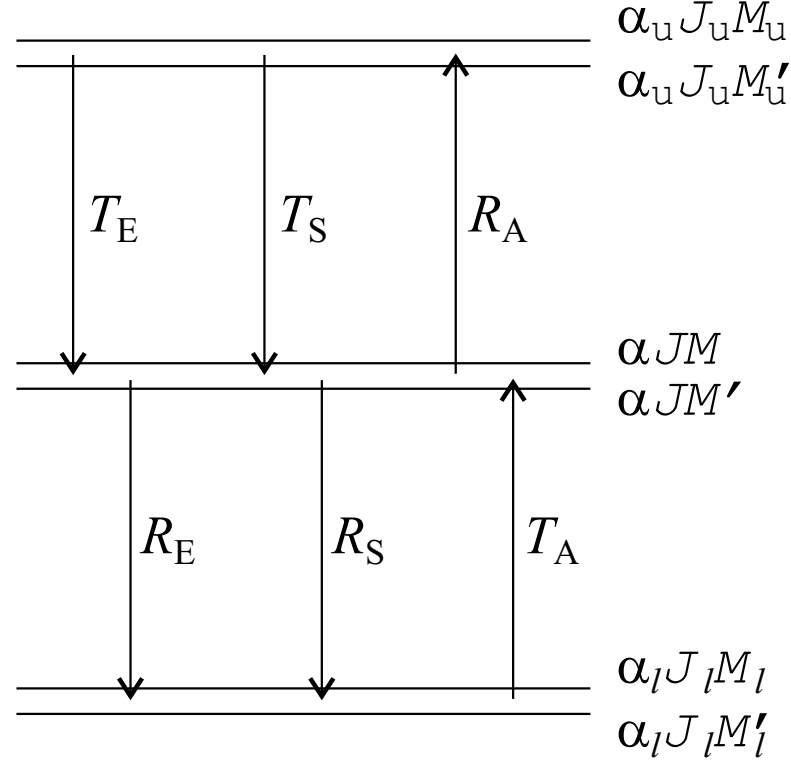

Figure 5.3: Grotrian diagram for the radiative processes contributing to the evolution of the density matrix elements.

where $T_{\mathrm{A}}, T_{\mathrm{E}}$, and $T_{\mathrm{S}}$ are the coherence-transfer rates caused by absorption from lower levels, by spontaneous emission from upper levels and by stimulated emission from upper levels, respectively (Fig. 5.3). The density matrix elements are increased due to these processes. The quantities $R_{\mathrm{A}}, R_{\mathrm{E}}$, and $R_{\mathrm{S}}$ are the coherence-relaxation rates caused by absorption, spontaneous and stimulated emission, respectively. The density matrix elements are decreased due to these processes. The effect of stimulated radiation emission (described by $T_{\mathrm{S}}$ and $R_{\mathrm{S}}$ ) can be neglected in the coronal plasma.

With help of the Wigner-Eckart theorem as for equations (5.16) and (5.25) one finds for the induced radiative processes:

$$
\begin{aligned}
& T_{\mathrm{A}}\left(\alpha J M M^{\prime}, \alpha_{l} J_{l} M_{l} M_{l}^{\prime}\right)=\left(2 J_{l}+1\right) B_{\alpha_{l} J_{l} \rightarrow \alpha J} \times \\
& \times 3(-1)^{M_{l}-M_{l}^{\prime}} \sum_{q q^{\prime}}\left(\begin{array}{ccc}
J & J_{l} & 1 \\
-M & M_{l} & -q
\end{array}\right)\left(\begin{array}{ccc}
J & J_{l} & 1 \\
-M^{\prime} & M_{l}^{\prime} & -q^{\prime}
\end{array}\right) J_{q q^{\prime}}\left(\nu_{\alpha J, \alpha_{l} J_{l}}\right), \\
& R_{\mathrm{A}}\left(\alpha J M M^{\prime}\right)=\frac{1}{2} \sum_{\alpha_{u} J_{u}}(2 J+1) B_{\alpha J \rightarrow \alpha_{u} J_{u}} \times \\
& \times 3(-1)^{M-M^{\prime}} \sum_{q q^{\prime} M_{u}}\left(\begin{array}{ccc}
J_{u} & J & 1 \\
-M_{u} & M & -q
\end{array}\right)\left(\begin{array}{ccc}
J_{u} & J & 1 \\
-M_{u} & M^{\prime} & -q^{\prime}
\end{array}\right) J_{q q^{\prime}}\left(\nu_{\alpha_{u} J_{u}, \alpha J}\right),
\end{aligned}
$$

and for spontaneous radiative processes:

$$
\begin{array}{r}
T_{\mathrm{E}}\left(\alpha J M M^{\prime}, \alpha_{u} J_{u} M_{u} M_{u}^{\prime}\right)=\left(2 J_{u}+1\right) A_{\alpha_{u} J_{u} \rightarrow \alpha J} \times \\
\times(-1)^{M_{u}-M_{u}^{\prime}} \sum_{q}\left(\begin{array}{ccc}
J_{u} & J & 1 \\
-M_{u}^{\prime} & M^{\prime} & -q
\end{array}\right)\left(\begin{array}{ccc}
J_{u} & J & 1 \\
-M_{u} & M & -q
\end{array}\right), \\
R_{\mathrm{E}}\left(\alpha J M M^{\prime}\right)=\frac{1}{2} \delta_{M M^{\prime}} \sum_{\alpha_{l} J_{l}} A_{\alpha J \rightarrow \alpha_{l} J_{l}},
\end{array}
$$


where

$$
J_{q q^{\prime}}(\nu)=\frac{1}{4 \pi} \int \sum_{i=0}^{3} \mathcal{T}_{q q^{\prime}}\left(i, \Omega^{\prime}\right) S_{i}\left(\nu, \Omega^{\prime}\right) d \Omega^{\prime}
$$

is the reducible radiation field tensor. Here, $S_{i}\left(\nu, \Omega^{\prime}\right)$ is the Stokes vector of the incident radiation field propagating in the direction $\Omega^{\prime}$.

The rates in (5.27) are due to radiative processes. To include the collisional excitation and de-excitation, it is possible to modify (5.27) within the impact approximation ( in which the duration of the collisional interaction is much smaller than the relaxation time due to radiation) by adding the collisional rates to the corresponding radiative ones (Lamb $\&$ ter Haar 1971).

\subsection{The role of the collisions}

There are two excitation mechanisms for ions in the corona: excitation by collisions and excitation by incident radiation coming from the photosphere.

Collisions (we consider only Fe XIII and Fe xIV, see figures 5.5 and 5.6) is the dominat exitation process in the lower corona near the photosphere (Sahal-Brechot 1974b). Due to the isotropic character of the collisions, they do not contribute to the polarization. Collisions can be divided in two types: those that are responsible for excitations between two different $J$-levels, and those that induce transitions between two magnetic sublevels within the same $J$-level (depolarizing collisions). The last type of collisions equilibrate the populations of the sublevels, a process which causes a depolarization of the emitted radiation.

Not only collisions for transitions within the line of interest must be taken into account while solving statistical equilibrium equation, but also collisions causing an exited configuration of an ion and subsequent cascades from the higher states must be considered (Pecker and Thomas 1962). The main mechanism for populating high exited levels of Fe XIV is the electron excitation from the ground state and the first exited levels (Petrini 1970).

On the other hand, depending on the impacting particles, collisions can be due to electron and proton impacts (the ion density in the corona is relatively low). Proton impact at coronal temperature can only excite transitions between sublevels of the ground configuration; excitations from the ground state to the exited configuration can be neglected (Landman 1975). For the Fe XIII and Fe XIV, depolarization rate caused by proton impact is very weak in comparison with electron excitation (Sahal-Brechot 1974a).

The numerical solution of the statistical equilibrium equation (5.27) for the density matrix elements was obtained by a number of authors (Sahal-Brechot 1974a,b, 1977; House 1977), who took into account the various collisional regimes listed above.

\subsection{Photo-excitation}

Beside collisional excitation, photo-excitation by radiation coming from the photosphere is another significant excitation process, especially at height above 1.4 solar radii (Raju et 
al. 1991). This process is responsible for the resonance scattering and Hanle (in presence of a magnetic field) effects.

The photospheric radiation from the area bounded by the cone $\Omega$ comes to the point $M$ situated at the distance $R$ from the Sun's center (Fig. 5.4). The radiation density at the photospheric surface according black body assumption is

$$
u(\nu)=\frac{8 \pi h \nu^{3}}{c^{3}} \frac{1}{\mathrm{e}^{h \nu / k T}-1},
$$

with radiation temperature $T \approx 5770 \mathrm{~K}$ (Allen 1973). The radiation density at the point $M$ (Van de Hulst 1950) is

$$
I(\nu)=W u(\nu)=\frac{2 A+B}{4} u(\nu),
$$

where $W$ is the limb-darkened total dilution. The terms $A$ and $B$ are defined through the center-limb darkening coefficient, $q$ (Van de Hulst 1950):

$$
\begin{array}{r}
A=(1-q) \frac{2(1-C)+\frac{1}{3}\left(1-C^{3}\right)}{2}+q \frac{4 D+E}{16}, \\
B=(1-q)\left(\frac{2}{3}-C+\frac{1}{3} C^{3}\right)+q \frac{4 D-E}{8},
\end{array}
$$

where

$$
\begin{array}{r}
D=1+\frac{C^{2}}{S} \ln \frac{C}{1+S}, \\
E=1+S^{2}+\frac{C^{4}}{S} \ln \frac{C}{1+S}, \\
S=\sin \Omega=R_{\odot} / R, C=\cos \Omega
\end{array}
$$

The radiation density, $I$, at the point $M$ can be splitted into an isotropic component, $I_{\mathrm{i}}$, and anisotropic one, $I_{\mathrm{a}},\left(I=I_{\mathrm{i}}+I_{\mathrm{a}}\right)$ :

$$
\begin{array}{r}
I_{\mathrm{i}}=\frac{3}{4} B u_{\nu}=W_{i} u_{\nu}, \\
I_{\mathrm{a}}=\frac{1}{2}(A-B) u_{\nu}=W_{a} u_{\nu},
\end{array}
$$

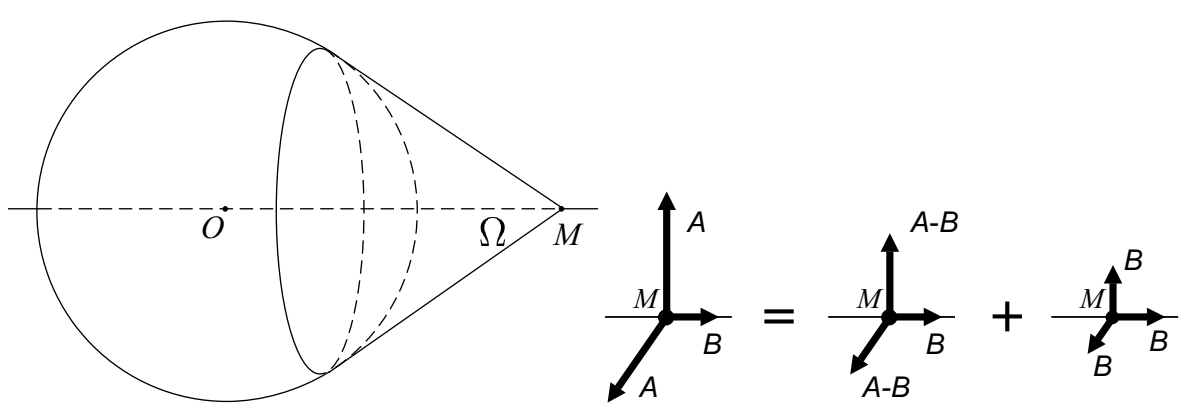

Figure 5.4: Anisotropy of the incident radiation field (Charvin 1965). 
where $W_{i}$ and $W_{a}$ are isotropic and anisotropic dilution factors, respectively. The absorption rate of incident radiation can also be splitted into two parts expressed through the Einstein coefficient for stimulated transition, $B_{\alpha^{\prime} J^{\prime} M^{\prime} \rightarrow \alpha J M}$ (Sahal-Brechot 1974b), an isotropic absorbtion rate

$$
B_{\alpha^{\prime} J^{\prime} M^{\prime} \rightarrow \alpha J M} \frac{\sigma_{i}}{3}
$$

and an anisotropic one, which creates the inequality of the populations of the Zeeman sublevels

$$
B_{\alpha^{\prime} J^{\prime} M^{\prime} \rightarrow \alpha J M} \frac{\sigma_{a}}{2} f(\Delta M, \Theta),
$$

where $\Delta M=M-M^{\prime}, \Theta$ is the angle between the quantization axis co-directed with $\boldsymbol{B}$ and the direction of the incident radiation, $\Omega^{\prime}$, (figure 5.2), and

$$
f(\Delta M, \Theta)=\left\{\begin{array}{l}
\sin ^{2} \Theta, \text { for } \Delta M=0 \\
\frac{1+\cos ^{2} \Theta}{2}, \text { for } \Delta M= \pm 1
\end{array}\right.
$$

\subsection{Density matrix in irreducible tensor representation}

The density matrix elements in (5.25) and (5.27) depend on the reference system. If the "new" reference system, $S_{n e w}$, is obtained from the "old" one, $S_{\text {old }}$, by the Euler rotation, $R$, then the density matrix transforms under the rule:

$$
\left(\rho_{\alpha J}\left(M, M^{\prime}\right)\right)_{S_{\text {new }}}=\sum_{N N^{\prime}} \mathcal{D}_{N M}^{J}(R)^{*} \mathcal{D}_{N^{\prime} M^{\prime}}^{J}(R)\left(\rho_{\alpha J}\left(N, N^{\prime}\right)\right)_{S_{\text {old }}}
$$

where $\mathcal{D}_{N M}^{J}(R)$ are rotation matrixes associated with the transformation $R$. This transformation involves the product of two rotation matrices. It is possible to construct linear combinations of these matrix elements in such way that the transformation $R$ will involve only one rotation matrix. Then the density matrix will be described by spherical tensors of rank $K$ (Landi 1984):

$$
\begin{gathered}
\rho_{Q}^{K}(\alpha J)=\sum_{M M^{\prime}}(-1)^{J-M} \sqrt{2 K+1}\left(\begin{array}{ccc}
J & J^{\prime} & K \\
M & -M^{\prime} & -Q
\end{array}\right) \rho_{\alpha J}\left(M, M^{\prime}\right), \\
\left(\rho_{Q}^{K}(\alpha J)\right)_{S_{\text {new }}}=\sum_{Q^{\prime}} \mathcal{D}_{Q^{\prime} Q}^{K}(R)^{*}\left(\rho_{Q^{\prime}}^{K}(\alpha J)\right)_{S_{\text {old }}},
\end{gathered}
$$

where, due to the properties of the $3-j$ symbol, $K=0, \ldots, 2 J$ and $Q=-K, \ldots, K$. If the density matrix is diagonal $\left(M^{\prime}=M\right)$, i.e. in the no-coherence case, then, because of condition $M^{\prime}-M=-Q, Q=0$, a moment of the form $\rho_{0}^{K}$ is a linear combination of populations. A moment of the form $\rho_{Q}^{K}$ with $Q \neq 0$ is a linear combination of coherences between states characterized by projections of the angular momentum differing by $Q$.

Expressions similar to (5.43) and (5.44) can be written for the geometric tensor:

$$
\begin{gathered}
\mathcal{T}_{Q}^{K}(i, \boldsymbol{\Omega})=\sum_{q q^{\prime}}(-1)^{1+q} \sqrt{3(2 K+1)}\left(\begin{array}{ccc}
1 & 1 & K \\
q & -q^{\prime} & -Q
\end{array}\right) \mathcal{T}_{q q^{\prime}}(i, \boldsymbol{\Omega}), \\
\left(\mathcal{T}_{Q}^{K}(i, \boldsymbol{\Omega})\right)_{S_{\text {new }}}=\sum_{Q^{\prime}} \mathcal{D}_{Q^{\prime} Q}^{K}(R)\left(\mathcal{T}_{Q^{\prime}}^{K}(i, \boldsymbol{\Omega})\right)_{S_{\text {old }}},
\end{gathered}
$$


where $q, q^{\prime}=0, \pm 1$ denote spherical coordinate components, and due to the properties of the 3 - $j$ symbol $0 \leq K \leq 2$ and $Q=-K, \ldots, K$.

For the $K=0$ rank moment of the density matrix we have

$$
\rho_{0}^{0}(\alpha J)=\frac{1}{\sqrt{2 J+1}} \sum_{M} \rho_{\alpha J}(M, M),
$$

which means that $\sqrt{2 J+1} \rho_{0}^{0}(\alpha J)$ is the population of the level $(\alpha J)$. The multipole moments of rank $K=1$ are connected with the average value of the angular momentum components. When these values are non-zero, there is a preferred direction in space, along which the atom is oriented. The multipole moments of rank $K=2$ are connected with the average value of bilinear combinations of the angular momentum components. These multipole moments are called the alignment components of the density matrix, and they characterize the inequality of populations between $\pi$ - and $\sigma$-components of the magnetic sublevels. The multipole moments of higher rank $n$ are connected with the average values of polynomials of degree $n$ in the angular momentum components.

\subsection{The non-coherence approximation}

There is the possibility of coherences between levels belonging to different atomic terms due to the level crossing. But, for Fe XIII and Fe xIv, the level crossing of different terms involves only highly excited states (never involves the ground one), which are excited and depopulated by isotropic collissional and spontateous emission processes (Sahal-Brechot 1977). Therefore, the magnetic levels are equally and incoherently populated within a level $(\alpha J)$, such that

$$
\rho\left(\alpha J M, \alpha^{\prime} J^{\prime} M^{\prime}\right)=\delta_{\alpha^{\prime} \alpha} \delta_{J J^{\prime}} \rho_{\alpha J}\left(M, M^{\prime}\right)
$$

Because of the low spontaneous transition probability, $A$, and the typical coronal magnetic field strength of $\sim 10 \mathrm{G}\left(\omega_{L} \gg A\right.$, strong field regime for Hanle-effect), any coherences between Zeeman sublevels are destroyed. Therefore, the off-diagonal components in the density matrix vanish:

$$
\rho_{\alpha J}\left(M, M^{\prime}\right)=\delta_{M M^{\prime}} \rho_{\alpha J}(M, M) .
$$

This approximation of completely diagonal density matrix leads to the restriction of the $Q$ components in the spherical statistical tensors $(Q=0)$ :

$$
\rho_{Q}^{K}(\alpha J)=\delta_{Q 0} \rho_{0}^{K}(\alpha J) .
$$

\subsection{Weak field approximation}

This section is devoted to considering the emission coefficient for the special case of the solar corona and based mainly on the investigation of Casini \& Judge (1999) and references therein.

Because of high temperature $\left(\sim 10^{6} \mathrm{~K}\right)$ and relatively weak magnetic field strength $(\sim 10 \mathrm{G})$, thermal broadening of the line profile is much larger than Zeeman splitting. 
Therefore it is possible to represent the Voigt profile in (5.25) by a Taylor expansion up to the first order in terms of the Zeeman splitting,

$$
\delta \omega_{M M_{0}}=\omega_{\mathrm{L}}\left(g_{\alpha_{0} J} M-g_{\alpha_{0} J_{0}} M_{0}\right) .
$$

The expansion about the center of gravity of the line yields: $\omega_{c}=\omega_{\alpha_{0} J, \alpha_{0} J_{0}}$ :

$$
\phi\left(\omega_{\alpha_{0} J M, \alpha_{0} J_{0} M_{0}}-\omega\right)=\phi\left(\omega_{c}-\omega\right)+\phi^{\prime}\left(\omega_{c}-\omega\right) \delta \omega_{M M_{0}},
$$

where $g_{\alpha_{0} J}$ and $g_{\alpha_{0} J_{0}}$ are the Lande factors of the levels $\left(\alpha_{0} J\right)$ and $\left(\alpha_{0} J_{0}\right)$, respectively. This representation suggests to divide the emission coefficients into two parts, zero- and first-order contribution ones:

$$
\begin{aligned}
& \varepsilon_{i}(\omega, \boldsymbol{\Omega})=\varepsilon_{i}^{(0)}(\omega, \boldsymbol{\Omega})+\varepsilon_{i}^{(1)}(\omega, \boldsymbol{\Omega}), \\
& \varepsilon_{i}^{(0)}(\omega, \Omega)=\frac{\hbar \omega}{4 \pi} N(2 J+1) A_{\alpha_{0} J \rightarrow \alpha_{0} J_{0}} \phi\left(\omega_{c}-\omega\right) \times \\
& \times \sum_{K}(-1)^{1+J+J_{0}} \sqrt{3}\left\{\begin{array}{ccc}
1 & 1 & K \\
J & J & J_{0}
\end{array}\right\} \rho_{0}^{K}\left(\alpha_{0} J\right) \mathcal{T}_{0}^{K}(i, \boldsymbol{\Omega}), \\
& \varepsilon_{i}^{(1)}(\omega, \boldsymbol{\Omega})=\omega_{\mathrm{L}} \frac{\hbar \omega}{4 \pi} N(2 J+1) A_{\alpha_{0} J \rightarrow \alpha_{0} J_{0}} \phi^{\prime}\left(\omega_{c}-\omega\right) \times \\
& \times \sum_{K K^{\prime}} \sqrt{3(2 K+1)\left(2 K^{\prime}+1\right)}\left(\begin{array}{ccc}
1 & K & K^{\prime} \\
0 & 0 & 0
\end{array}\right) \rho_{0}^{K}\left(\alpha_{0} J\right) \mathcal{T}_{0}^{K^{\prime}}(i, \Omega) \times \\
& \times\left((-1)^{J-J_{0}} g_{\alpha_{0} J} \sqrt{J(J+1)(2 J+1)}\left\{\begin{array}{ccc}
1 & K & K^{\prime} \\
J & J & J
\end{array}\right\}\left\{\begin{array}{ccc}
1 & 1 & K^{\prime} \\
J & J & J_{0}
\end{array}\right\}-\right. \\
& \left.-g_{\alpha_{0} J_{0}} \sqrt{J_{0}\left(J_{0}+1\right)\left(2 J_{0}+1\right)}\left\{\begin{array}{ccc}
1 & K & K^{\prime} \\
J_{0} & J & 1 \\
J_{0} & J & 1
\end{array}\right\}\right)
\end{aligned}
$$

Here, the two-row matrix form expressions in curved brackets are Wigner $6 j$-symbols, and the three-row matrix form expression in curved brackets is an Wigner $9 j$-symbol.

It is easy to find from (5.54) and (5.55) how different multiples of the density matrix contribute to the emission coefficients (Tables 5.1 and 5.2). Atomic orientation $(K=1)$ can be neglected in the solar corona because the exciting radiation from the photosphere is unpolarized. Also the contribution from the $K=3$ moment can be neglected, because it has no observational effect. Thus, linear polarization can be produced only by atomic alignment $(K=2)$. And, total level population and atomic alignment also contribute to the circular polarization.

Finally, introducing the alignment factor

$$
\sigma_{Q}^{K}\left(\alpha_{0} J\right)=\frac{\rho_{Q}^{K}\left(\alpha_{0} J\right)}{\rho_{0}^{0}\left(\alpha_{0} J\right)},
$$

and the effective Lande factor for the transition $\left(\alpha_{0} J\right) \rightarrow\left(\alpha_{0} J_{0}\right)$ (Landi 1982)

$$
\bar{g}=\frac{g_{\alpha_{0} J}+g_{\alpha_{0} J_{0}}}{2}+\frac{g_{\alpha_{0} J}-g_{\alpha_{0} J_{0}}}{4}\left(J(J+1)-J_{0}\left(J_{0}+1\right)\right),
$$




\begin{tabular}{|c|c|c|c|c|}
\hline$i$ & $K$ & $\mathcal{T}_{0}^{K}(i, \boldsymbol{\Omega})$ & $K^{\prime}$ & $\mathcal{T}_{0}^{K^{\prime}}(i, \boldsymbol{\Omega})$ \\
\hline \multirow{5}{*}{0} & 0 & 0 & 1 & 0 \\
\hline & \multirow{2}{*}{1} & \multirow{2}{*}{0} & $\overline{0}$ & 0 \\
\hline & & & 2 & $\frac{1}{2 \sqrt{2}}\left(3 \cos ^{2} \theta-1\right)$ \\
\hline & 2 & $\frac{1}{2 \sqrt{2}}\left(3 \cos ^{2} \theta-1\right)$ & 1 & 0 \\
\hline & 3 & - & 2 & $\frac{1}{2 \sqrt{2}}\left(3 \cos ^{2} \theta-1\right)$ \\
\hline \multirow{5}{*}{1} & 0 & 0 & 1 & $\begin{array}{l}0 \\
0\end{array}$ \\
\hline & 1 & \multirow[b]{2}{*}{0} & 0 & 0 \\
\hline & 1 & & 2 & $\frac{3}{2 \sqrt{2}} 3 \cos 2 \gamma \sin ^{2} \theta$ \\
\hline & 2 & $\frac{3}{2 \sqrt{2}} 3 \cos 2 \gamma \sin ^{2} \theta$ & 1 & 0 \\
\hline & 3 & - & 2 & $\frac{3}{2 \sqrt{2}} 3 \cos 2 \gamma \sin ^{2} \theta$ \\
\hline \multirow{5}{*}{2} & 0 & 0 & 1 & $\begin{array}{l}0 \\
\end{array}$ \\
\hline & \multirow{2}{*}{1} & \multirow{2}{*}{0} & 0 & 0 \\
\hline & & & 2 & $\frac{-3}{2 \sqrt{2}} 3 \sin 2 \gamma \sin ^{2} \theta$ \\
\hline & 2 & $\frac{-3}{2 \sqrt{2}} 3 \sin 2 \gamma \sin ^{2} \theta$ & 1 & 0 \\
\hline & 3 & - & 2 & $\frac{-3}{2 \sqrt{2}} 3 \sin 2 \gamma \sin ^{2} \theta$ \\
\hline \multirow{5}{*}{3} & 0 & 0 & 1 & $\sqrt{\frac{3}{2}} \cos \theta$ \\
\hline & \multirow{2}{*}{1} & \multirow{2}{*}{$\sqrt{\frac{3}{2}} \cos \theta$} & 0 & 0 \\
\hline & & & 2 & 0 \\
\hline & 2 & 0 & 1 & $\sqrt{\frac{3}{2}} \cos \theta$ \\
\hline & 3 & - & 2 & 0 \\
\hline
\end{tabular}

Table 5.1: The values of the spherical tensors $\mathcal{T}_{0}^{K}$ and $\mathcal{T}_{0}^{K^{\prime}}$ for all possible values of $K$ and $K^{\prime}$ and non-zero values of the $3-j$ symbol in (5.54) and (5.55).

\begin{tabular}{|c|c|c|}
\hline$i$ & $\varepsilon_{i}^{(0)}(\omega, \boldsymbol{\Omega})$ & $\varepsilon_{i}^{(1)}(\omega, \boldsymbol{\Omega})$ \\
\hline 0, Stokes- $I$ & $K=0,2$ & $K=1,3$ \\
1, Stokes- $Q$ & $K=2$ & $K=1,3$ \\
2, Stokes- $U$ & $K=2$ & $K=1,3$ \\
3, Stokes- $V$ & $K=1$ & $K=0,2$ \\
\hline
\end{tabular}

Table 5.2: Contributing components, $K$, of the density matrix tensor, $\rho_{0}^{K}\left(\alpha_{0} J\right)$, to the emission coefficients 
we get expressions for the emission coefficients:

$$
\begin{gathered}
\varepsilon_{I}(\omega, \Omega)=\varepsilon_{0}^{(0)}(\omega, \Omega)=\frac{\hbar \omega}{4 \pi} N_{\alpha_{0} J} A \phi(\omega)\left(1+D_{J J_{0}} \sigma_{0}^{2}\left(\alpha_{0} J\right) \frac{3 \cos ^{2} \theta-1}{2 \sqrt{2}}\right) \\
\varepsilon_{Q}(\omega, \boldsymbol{\Omega})=\varepsilon_{1}^{(0)}(\omega, \boldsymbol{\Omega})=\frac{\hbar \omega}{4 \pi} N_{\alpha_{0} J} A \phi(\omega) D_{J J_{0}} \sigma_{0}^{2}\left(\alpha_{0} J\right) \frac{3}{2 \sqrt{2}} \cos 2 \gamma \sin ^{2} \theta \\
\varepsilon_{U}(\omega, \boldsymbol{\Omega})=\varepsilon_{2}^{(0)}(\omega, \boldsymbol{\Omega})=-\frac{\hbar \omega}{4 \pi} N_{\alpha_{0} J} A \phi(\omega) D_{J J_{0}} \sigma_{0}^{2}\left(\alpha_{0} J\right) \frac{3}{2 \sqrt{2}} \sin 2 \gamma \sin ^{2} \theta \\
\varepsilon_{V}(\omega, \boldsymbol{\Omega})=\varepsilon_{3}^{(1)}(\omega, \boldsymbol{\Omega})=-\omega_{L} \frac{\hbar \omega}{4 \pi} N_{\alpha_{0} J} A \phi^{\prime}(\omega)\left(\bar{g}+E_{J J_{0}} \sigma_{0}^{2}\left(\alpha_{0} J\right)\right) \cos \theta
\end{gathered}
$$

where

$$
\begin{gathered}
D_{J J_{0}}=(-1)^{1+J+J_{0}} \sqrt{3(2 J+1)}\left\{\begin{array}{ccc}
1 & 1 & 2 \\
J & J & J_{0}
\end{array}\right\}, \\
\times\left((-1)^{J-J_{0}} g_{\alpha_{0} J} \sqrt{J(J+1)(2 J+1)}\left\{\begin{array}{ccc}
1 & 2 & 1 \\
J & J & J
\end{array}\right\}\left\{\begin{array}{lll}
1 & 1 & 1 \\
J & J & J_{0}
\end{array}\right\}-\right. \\
\left.-g_{\alpha_{0} J_{0}} \sqrt{J_{0}\left(J_{0}+1\right)\left(2 J_{0}+1\right)}\left\{\begin{array}{ccc}
1 & 2 & 1 \\
J_{0} & J & 1 \\
J_{0} & J & 1
\end{array}\right\}\right) .
\end{gathered}
$$

Here $N_{\alpha_{0} J}$ is the population density of the exited level, and $A$ the Einstein spontaneous coefficient for the $\left(\alpha_{0} J_{0}\right) \rightarrow\left(\alpha_{0} J\right)$ transition.

We can clearly see from the (5.58) that total emission vector, $\varepsilon_{I}$, depends on the LOS direction, and how strong this dependence is defined by the alignment factor $\sigma_{0}^{2}$. When $\sigma_{0}^{2}\left(\alpha_{0} J\right)$ is much less than unity (for example, $\sigma_{0}^{2}\left(\alpha_{0} J\right) \sim 10^{-2}$ for the He I D3 line), we can neglect the directional dependence of the total emission coefficient.

\subsection{Magnetograph formula}

From equations (5.58)-(5.61) one can see that total emission and Stokes- $V$ component are connected via magnetograph formula:

$$
\varepsilon_{V}(\omega, \boldsymbol{\Omega})=k \bar{g} \omega_{L} \cos \theta \frac{d \varepsilon_{I}(\omega, \boldsymbol{\Omega})}{d \omega} .
$$

The effect of the alignment is represented here by the coefficient

$$
k=\frac{1+E_{J J_{0}} \sigma_{0}^{2}\left(\alpha_{0} J\right) / g}{1+2^{-3 / 2} D_{J J_{0}} \sigma_{0}^{2}\left(\alpha_{0} J\right)\left(3 \cos ^{2} \theta-1\right)} .
$$

Again, like in the case for the total emissivity, this coefficient, $k$, depends on the alignment factor $\sigma_{0}^{2}$. Integrating (5.64) along some LOS we get

$$
V(\omega, \boldsymbol{\Omega})=\int \varepsilon_{V}(\omega, \boldsymbol{\Omega}) d l=\int k \bar{g} \omega_{L} \cos \theta \frac{d \varepsilon_{I}(\omega, \boldsymbol{\Omega})}{d \omega} d l
$$




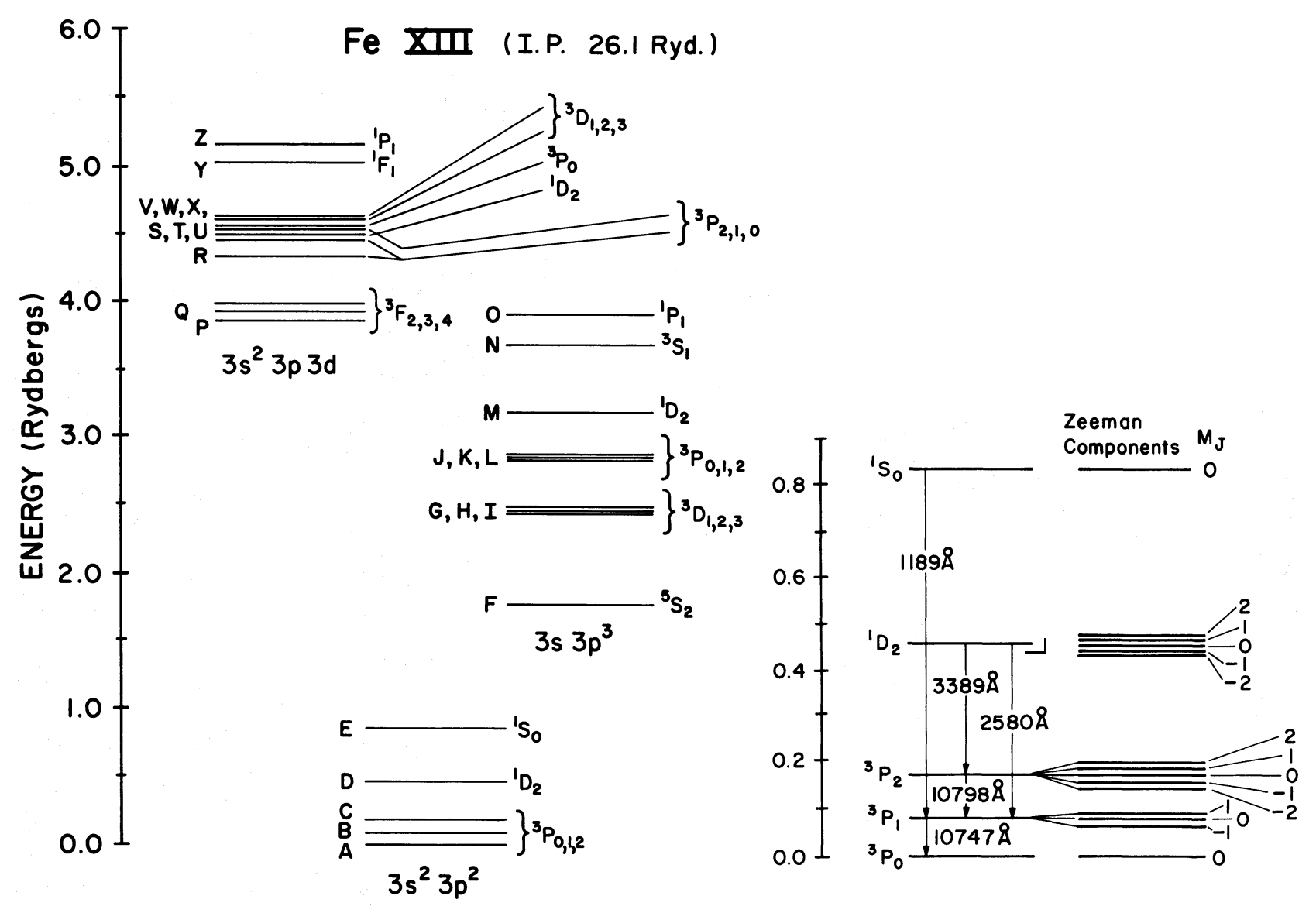

Figure 5.5: Energy levels of Fe XIII. The Zeeman splitting is only shown for $3 \mathrm{~s}^{2} 3 \mathrm{p}^{2}$ term (House 1977).

Here, the derivative of the total emission, $I$, which is a quantity that can independently be measured as a LOS integral:

$$
\frac{d I(\omega, \boldsymbol{\Omega})}{d \omega}=\int\left(\frac{d \varepsilon_{I}(\omega, \boldsymbol{\Omega})}{d \omega}\right) d l
$$

The nice property of using the magnetograph formula, in contrary to the Faraday-effect, is that it is not necessary to know the distribution of the ion density and temperature over the corona. All coefficients in (5.64) are either intrinsically atomic quantities which depend only on the type of ion, while $d \varepsilon_{I}(\omega, \Omega) / d \omega$ can be obtained from a scalar tomographic inversion of the observations (5.67).

\subsection{Emission line $10747 \AA$ of the $\mathrm{Fe}$ xIII}

The coronal emission line $10747 \AA$ was first observed by B. Lyot (Lyot 1939) in 1936. Later, the line was identified by B. Edlén (1943) as the ${ }^{3} \mathrm{P}_{1}-{ }^{3} \mathrm{P}_{0}$ transition of the ground configuration of the Fe XIII ion (Fig. 5.5). First measurements of the polarization of the line were made by Eddy and Malville (1967) during the 1965 eclipse. The observations showed that the linear polarization was predominantly radially oriented and the degree 
of polarization increased with distance from the Sun. Later, the Coronal Emission Line Polarimeter (KELP) constructed by the High Altitude Observatory (HAO) provided more precise measurements (Querfeld \& Elmore 1976; Querfeld \& Smartt 1984). The theory for the interpretation of the observation was also developed by Charvin (1965), House (1974, 1977), Querfeld (1982).

Since the line is infrared it is very promising for the derivation of the magnetic field by the longitudinal Zeeman-effect, because the Zeeman splitting is proportional to the second power of the wavelength, but the thermal broadening increases only with the first power. Moreover, this line has lower and upper states $J_{l}=0$ and $J_{u}=1$, respectively. The upper state therefore splits only into three magnetic sublevels which simplifies the calculations.

As the levels of interest belong to the same ground state, we can omit $\alpha$ in the following notations for the density matrix. Taking into account that $J_{l}=0$ and $J_{u}=1$, we write for the sublevels with $M= \pm 1$ :

$$
\rho_{1}(1)=\rho_{1}(-1)=\rho_{1}( \pm 1) \equiv \rho_{\alpha J_{u}}( \pm 1),
$$

and for the sublevel with $M=0$

$$
\rho_{1}(0) \equiv \rho_{\alpha J_{u}}(0)
$$

The emission coefficients (5.25) into space angle $\Omega$ can be integrated over the line profile and are then after (Querfeld 1982)

$$
\left(\begin{array}{l}
\varepsilon_{I}(\boldsymbol{\Omega}) \\
\varepsilon_{Q}(\boldsymbol{\Omega}) \\
\varepsilon_{U}(\boldsymbol{\Omega}) \\
\varepsilon_{V}(\boldsymbol{\Omega})
\end{array}\right)=\frac{3 \hbar \omega A}{8 \pi}\left(\begin{array}{c}
\rho_{1}( \pm 1)\left(1+\cos ^{2} \theta\right)+\rho_{1}(0) \sin ^{2} \theta \\
{\left[\rho_{1}( \pm 1)-\rho_{1}(0)\right] \sin ^{2} \theta \cos 2 \alpha} \\
{\left[\rho_{1}( \pm 1)-\rho_{1}(0)\right] \sin ^{2} \theta \sin 2 \alpha} \\
0
\end{array}\right)
$$

Here $\alpha$ now denotes the angle between the local radius vector and the observed polarization projected on the plane of the sky (POS) (Fig. 5.2). Let us denote the sum and difference of the populations through

$$
\begin{gathered}
\Sigma=\left[2 \rho_{1}( \pm 1)+\rho_{1}(0)\right], \\
\Delta=\left[\rho_{1}( \pm 1)-\rho_{1}(0)\right],
\end{gathered}
$$

Then the emission coefficients are

$$
\left(\begin{array}{c}
\varepsilon_{I}(\boldsymbol{\Omega}) \\
\varepsilon_{Q}(\boldsymbol{\Omega}) \\
\varepsilon_{U}(\boldsymbol{\Omega})
\end{array}\right)=\frac{\hbar \omega A}{8 \pi}\left(\begin{array}{c}
2 \Sigma+\Delta\left(3 \cos ^{2} \theta-1\right) \\
3 \Delta \sin ^{2} \theta \cos 2 \alpha \\
3 \Delta \sin ^{2} \theta \sin 2 \alpha
\end{array}\right) .
$$

Solving the statistical equilibrium equations (5.27), one finds that $\Delta$ is proportional to $\left(3 \cos ^{2} \Theta-1\right)$ where $\Theta$ is the angle between the direction of the radius vector from the center of the Sun and the magnetic field vector (Charvin 1965; Sahal-Brechot 1974a, 1977; House 1977). The $\Sigma$ and $\Delta$ can be decomposed in the manner (Querfeld 1982):

$$
\Sigma=\frac{\Sigma}{\rho_{0}(0)} \frac{\rho_{0}(0)}{N_{\mathrm{FeXIII}}} \frac{N_{\mathrm{FeXIII}}}{N_{\mathrm{Fe}}} \frac{N_{\mathrm{Fe}}}{N_{\mathrm{H}}} \frac{N_{\mathrm{H}}}{N_{e}} N_{e}=\Sigma^{\prime} \rho_{0}^{\prime}(0) N_{i} A_{\mathrm{Fe}} \frac{N_{\mathrm{H}}}{N_{e}} N_{e},
$$




$$
\Delta=\frac{\Delta}{\rho_{0}(0)} \frac{\rho_{0}(0)}{N_{\mathrm{FeXIII}}} \frac{N_{\mathrm{FeXIII}}}{N_{\mathrm{Fe}}} \frac{N_{\mathrm{Fe}}}{N_{\mathrm{H}}} \frac{N_{\mathrm{H}}}{N_{e}} N_{e}=\Delta^{\prime}\left(3 \cos ^{2} \Theta-1\right) \rho_{0}^{\prime}(0) N_{i} A_{\mathrm{Fe}} \frac{N_{\mathrm{H}}}{N_{e}} N_{e}
$$

where $\rho_{0}(0)$ is the population of the level $J=0$, and $\rho_{0}^{\prime}(0)=\rho_{0}(0) / N_{\mathrm{FeXIII}}$ is the population relative to the total Fe XIII number density, $N_{\mathrm{FeXIII}}$. the symbol $N_{\mathrm{Fe}}$ is the iron number density, $N_{\mathrm{H}}$ the proton number density, and $N_{e}$ the electron density. The iron abundance $A_{\mathrm{Fe}}=N_{\mathrm{Fe}} / N_{\mathrm{H}}$ is assumed to be $7.08 \cdot 10^{-5}$ (Withbroe 1971). The value of the ionization equilibrium $N_{i}=N_{\mathrm{FeXIII}} / N_{\mathrm{Fe}}$ is 0.239 at temperature $T=1.8 \cdot 10^{6} \mathrm{~K}$ (Jacobs et al. 1977). The helium abundance is assumed to be $10 \%$, and so the proton abundance $N_{\mathrm{H}} / N_{e}$ is 0.8 .

The dependence of the population difference $\Delta$ on the angle $\Theta$ is the so called van Vleck effect (van Vleck 1925). The van Vleck effect completely depolarizes the scattered radiation when $\Theta^{*}=\arccos (1 / \sqrt{3}) \approx 54.7^{\circ}$ (the van Vleck angle). After the van Vleck angle is passed (i.e. for $\Theta>\Theta^{*}$ ), the Stokes vectors $Q$ and $U$ change sign, which means that the plane of polarization now is perpendicular to the magnetic field.

To calculate the quantities $\Sigma^{\prime}, \Delta^{\prime}$ and $\rho_{0}^{\prime}(0)$ Querfeld (1982) reduced the House (1977) statistical equilibrium equations to a set of 15 equations for all of the ground term sublevels by removing the explicit calculation of the 91 excited term sublevels. This set of 15 equations was further reduced to 10 equations because of the alignment property $\rho_{1}(M)=\rho_{1}(-M)$. Solving this reduced system of equations, he approximated expressions for the $\Sigma^{\prime}, \Delta^{\prime}$ and $\rho_{0}^{\prime}(0)$ by the fits:

$$
\begin{array}{r}
\rho_{0}^{\prime}(0) \frac{h \nu A R_{\odot}^{2}}{8 \pi I_{0}} N_{i} A_{\mathrm{Fe}} \frac{N_{\mathrm{H}}}{N_{e}}= \\
=250\left(1-0.360 W+0.0588 W^{2}\right) \frac{1+\left(0.00802+0.00214 \log T_{6}\right) N_{8}}{1+\left(0.0831+0.0394 \log T_{6}\right) N_{8}} \\
\Sigma^{\prime}=0.360 W+\frac{\left(0.0464+0.01013 \log T_{6}\right) N_{8}}{1+0.0178 N_{8}} \\
\Delta^{\prime}=0.0900 W_{a} \frac{1-\left(0.01172-0.00755 \log T_{6}\right) N_{8}}{1+0.0819 N_{8}},
\end{array}
$$

where

$$
1 \leq T_{6} \leq 3, \quad N_{8} \leq 100 .
$$

Here, $W$ and $W_{a}$ are the limb-darkened total and anisotropy dilution factors as defined by (5.34) and (5.34), with limb-darkening function $q=0.33$ (Allen 1973). In the above equations $T_{6}$ is the temperature in units of $10^{6} \mathrm{~K}, N_{8}$ is the electron density in units of $10^{8} \mathrm{~cm}^{-3}$, and $I_{0}$ is the central disk brightness.

\subsection{Emission line $5303 \AA$ of the $\mathrm{Fe} x \mathrm{xI}$}

The $5303 \AA$ line of Fe xIV (green line) is the brightest forbidden emission line in the visible coronal spectrum. The line was discovered by Young and Harkness during the 1869 total solar eclipse, and identified later by Grotrian (1939) and Edlen (1943). The emissivity of the green line has a maximum at $\sim 2 \cdot 10^{6} \mathrm{~K}$ (Esser et al. 1995; Guhathakurta et al. 1992). The excitation mechanism is mainly collisional up to $\sim 1.4 R_{\odot}$, while in the higher regions, the radiative excitation is dominant (Raju 1991). The line was intensively 


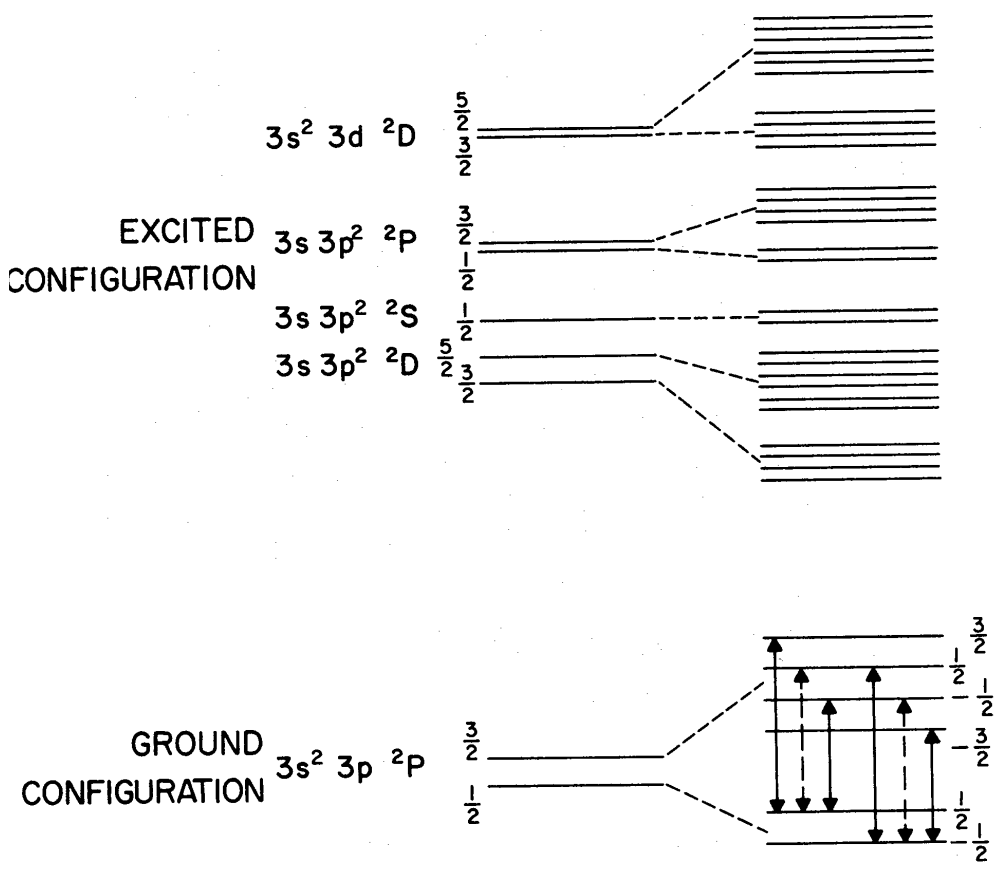

Figure 5.6: Energy levels of Fe XIV (House 1982).

observed by the LASCO coronograph on board the SOHO spacecraft (Brueckner et al. 1995; Schwenn et al. 1997; Inhester et al. 1999).

The observed green line emission is closely related to the underlying photospheric magnetic field. The brightest emissions come from active regions with strong magnetic field. A prominent feature at low solar activity are high-altitude streamers which occur in the regions where the magnetic flux from large active regions is connected to the highlatitude unipolar fields. The global emission pattern rotates quasi-rigidly at the rate of the dominant active regions.

A current-free magnetic field model does not always properly reflect the observed coronal structures, particularly near prominences and in the boundary region between open and closed magnetic fields (Wang et al. 1997).

One of the first polarization observation of the line was made by Mogilevskij et al. (1960) during the eclipse of June 30, 1954. Later, a coronameter especially designed to measure the green line polarization has been built at Pic-du-Midi observatory (Charvin 1971; Arnaud 1982a). The obtained polarization maps often display a large-scale homogeneous structure that is surprising because of the complex density structure of the corona (Arnaud 1982b).

As the levels of the line emission belong to the same ground configuration (Fig. 5.6), we can omit $\alpha$ in the following notations for the density matrix, and taking into acount that $J_{l}=\frac{1}{2}$ and $J_{u}=\frac{3}{2}$, we can write for the magnetic sublevels $M= \pm \frac{1}{2}$ of the lower state $J_{l}=\frac{1}{2}$

$$
\rho_{\frac{1}{2}}\left(-\frac{1}{2}\right)=\rho_{\frac{1}{2}}\left(\frac{1}{2}\right)=\rho_{\frac{1}{2}}\left( \pm \frac{1}{2}\right) \equiv \rho_{\alpha J_{l}}\left( \pm \frac{1}{2}\right),
$$


and for the magnetic sublevels $M= \pm \frac{1}{2}, \pm \frac{3}{2}$ of the upper state $J_{u}=\frac{3}{2}$

$$
\begin{gathered}
\rho_{\frac{3}{2}}\left(-\frac{1}{2}\right)=\rho_{\frac{3}{2}}\left(\frac{1}{2}\right)=\rho_{\frac{3}{2}}\left( \pm \frac{1}{2}\right) \equiv \rho_{\alpha J_{u}}\left( \pm \frac{1}{2}\right), \\
\rho_{\frac{3}{2}}\left(-\frac{3}{2}\right)=\rho_{\frac{3}{2}}\left(\frac{3}{2}\right)=\rho_{\frac{3}{2}}\left( \pm \frac{3}{2}\right) \equiv \rho_{\alpha J_{u}}\left( \pm \frac{3}{2}\right) .
\end{gathered}
$$

The sum and difference of the magnetic sublevel belonging to the upper level can be expressed as:

$$
\begin{aligned}
& \Sigma=2\left(\rho_{\frac{3}{2}}\left( \pm \frac{3}{2}\right)+\rho_{\frac{3}{2}}\left( \pm \frac{1}{2}\right)\right), \\
& \Delta=2\left(\rho_{\frac{3}{2}}\left( \pm \frac{3}{2}\right)-\rho_{\frac{3}{2}}\left( \pm \frac{1}{2}\right)\right) .
\end{aligned}
$$

Then the emission coefficients integrated over line profile are (House et al. 1982)

$$
\left(\begin{array}{c}
\left.\varepsilon_{I}(\boldsymbol{\Omega})\right) \\
\varepsilon_{Q}(\boldsymbol{\Omega}) \\
\varepsilon_{U}(\boldsymbol{\Omega})
\end{array}\right)=\frac{\hbar \omega A}{8 \pi}\left(\begin{array}{c}
4 \Sigma+\Delta\left(3 \cos ^{2} \theta-1\right) \\
3 \Delta \sin ^{2} \theta \cos 2 \alpha \\
3 \Delta \sin ^{2} \theta \sin 2 \alpha
\end{array}\right) .
$$

Introducing the relative sum and difference populations $\Sigma^{\prime}$ and $\Delta^{\prime}$ like in Sec. 5.10, we may write

$$
\begin{array}{r}
\Sigma=N \Sigma^{\prime}, \\
\Delta=N \Delta^{\prime}\left(3 \cos ^{2} \Theta-1\right),
\end{array}
$$

where $N$ is the total population of all sublevels in the ground configuration, and the equation (5.83) can be rewritten as

$$
\left(\begin{array}{c}
\left.\varepsilon_{I}(\boldsymbol{\Omega})\right) \\
\varepsilon_{Q}(\boldsymbol{\Omega}) \\
\varepsilon_{U}(\boldsymbol{\Omega})
\end{array}\right)=\frac{\hbar \omega A}{8 \pi} N\left(\begin{array}{c}
4 \Sigma^{\prime}+\Delta^{\prime}\left(3 \cos ^{2} \theta-1\right)\left(3 \cos ^{2} \Theta-1\right) \\
3 \Delta^{\prime}\left(3 \cos ^{2} \Theta-1\right) \sin ^{2} \theta \cos 2 \alpha \\
3 \Delta^{\prime}\left(3 \cos ^{2} \Theta-1\right) \sin ^{2} \theta \sin 2 \alpha
\end{array}\right) .
$$

For calculating the density matrix elements, House (1974) and Sahal-Brechot (1974b) used a nine level (34 sublevel) model of the Fe XIV (Fig. 5.6), i.e., the statistical equilibrium condition involves 34 equations. To reduce the calculation time, House et al. (1982) reduced these 34 equations to a set of six equations by reducing the equations which couple the ground to excited configurations to an equivalent set of indirect rates coupling only ground configuration sublevels. Using the alignment assumption $\rho_{\alpha J}(M)=\rho_{\alpha J}(-M)$, they further reduced the remaining six equations to three. These three equations give expressions for $\Sigma^{\prime}$ and $\Delta^{\prime}$ :

$$
\begin{array}{r}
\Sigma^{\prime}=\frac{W+0.6585\left(1-0.0697 T_{6}\right) N_{8}}{37.26+W+1.0295\left(1-0.0697 T_{6}\right) N_{8}}, \\
\Delta^{\prime}=\frac{W_{a}\left(1-\Sigma^{\prime}\right)}{149.04+\left(4.7420-0.1340 T_{6}\right) N_{8}},
\end{array}
$$

where $W$ and $W_{a}$ are the total and anisotropic dilution factors, respectively, which can be calculated using Allen's (1973) limb darkening function with $q=0.62$ (see Sec. 5.5). In these formulae, $T_{6}$ is the electron temperature in units of $10^{6} \mathrm{~K}$, and $N_{8}$ is the electron density in units of $10^{8} \mathrm{~cm}^{-3}$. 
The total population of all the sublevels in the ground configuration, $N$, can be assumed to be equal the total Fe xIV population, since the total population in the excited configuration is $\sim 10^{-9} N$. So, using the same notations like in Section 5.10, we have

$$
N=N_{\mathrm{FeXIV}}=N_{\mathrm{H}} A_{\mathrm{Fe}} \frac{N_{\mathrm{FeXIV}}}{N_{\mathrm{Fe}}} .
$$

\subsection{Influence of the alignment factor}

The total intensity (Stokes- $I$ ) in equations (5.86), (5.71) and (5.58) is composed of two terms. The first, proportional to $\Sigma^{\prime}$, is isotropic while the second, proportional to $\Delta^{\prime}$, depends on the angles $\theta$ and $\Theta$ which the local magnetic field forms with the LOS direction and the local radius vector, respectively. A measure of how much the Stokes- $I$ component deviates from isotropy is therefore given by the ratios:

$$
\begin{aligned}
& \eta_{\mathrm{FeXIII}}=\frac{\Delta_{\mathrm{FeXIII}}^{\prime}}{2 \Sigma_{\mathrm{FeXIII}}^{\prime}} \text { for } 10747 \AA \text { line of Fe XIII, } \\
& \eta_{\mathrm{FeXIV}}=\frac{\Delta_{\mathrm{FeXIV}}^{\prime}}{4 \Sigma_{\mathrm{FeXIV}}^{\prime}} \text { for } 5303 \AA \text { line of Fe XIV. }
\end{aligned}
$$

The population difference $\Delta$ is proportional to the alignment factor (5.56) which is different for different lines and varies with density and temperature.

Taking the coronal electron density distribution from Newkirk (1970),

$$
N_{e}(r)=\left(755 r^{-5.353}-168 r^{-14.738}+103800 r^{-20.446}\right) \cdot 10^{11} \mathrm{~cm}^{-3},
$$

where distance $r$ is in solar radii, and using (5.74)-(5.76) and (5.87)-(5.88), we can calculate the ratios (5.90) and (5.91) as functions of radial distance, $r$, and temperature, $T$ (Fig. 5.7).

To estimate the influence of the alignment factor in the total intensity, it is necessary to multiply the ratio $\eta$ with the term depending on the magnetic field configuration, $V(\theta) V(\Theta)=\left(3 \cos ^{2} \theta-1\right)\left(3 \cos ^{2} \Theta-1\right)$. Theoretically, $V(\theta) V(\Theta)$ varies within
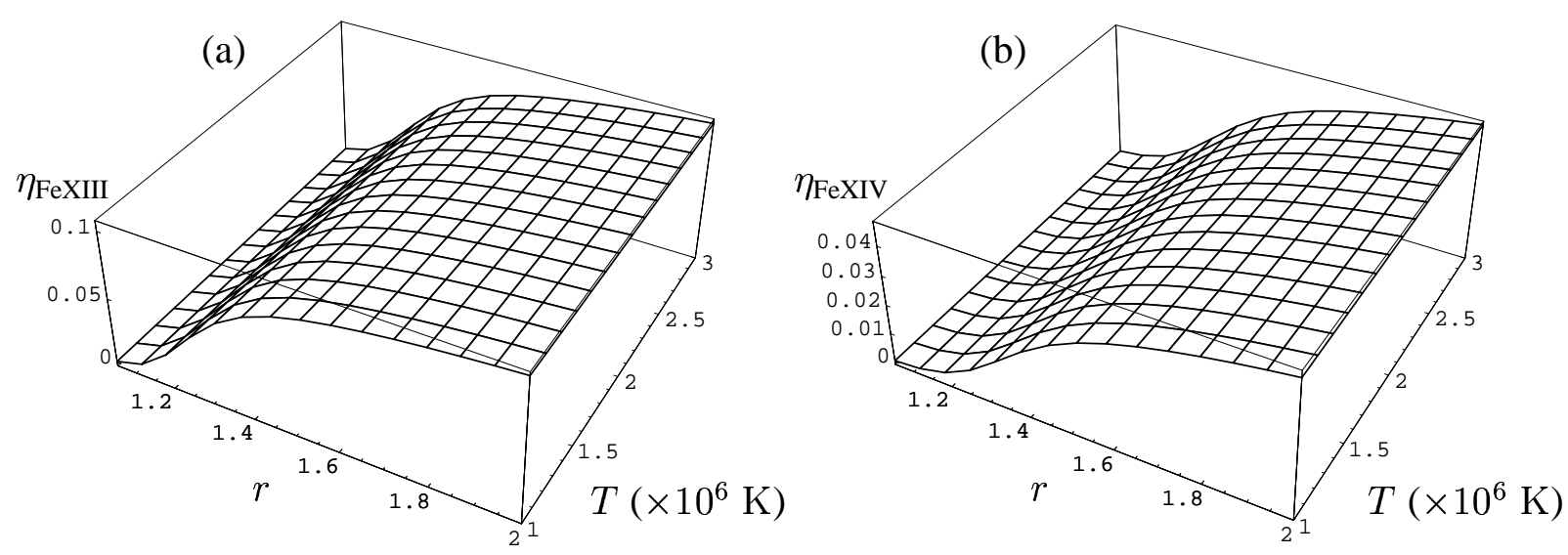

Figure 5.7: The ratios $\eta_{\text {FeXIII }}$ (a) and $\eta_{\text {FeXIV }}$ (b) for the $10747 \AA$ and $5303 \AA$ lines of Fe XIII and Fe XIV, respectively. 
$-2 \leq V(\theta) V(\Theta) \leq 4$. But because of central occulter (Sun's disk) we have a limitation in the possible LOS directions, and, therefore, $-2<V(\theta) V(\Theta) \lesssim 3.2$ for $R_{\odot}<r \leq 2 R_{\odot}$. So, depending on the magnetic field configuration, location of the observed region and LOS direction, the maximal contribution of the anisotropic part to the total intensity is less than 32 and $13 \%$ for the 10747 and $5303 \AA$ lines, respectively. This is the maximum error of a simplified emission coefficient $\varepsilon_{I}$ for Stokes- $I$ which omits the anisotropic part of the emission. The advantage of this simplified emission $\varepsilon_{I}$ is that it is depends linearly on $N \Sigma^{\prime}$ with a space-independent coefficient. Hence, $N \Sigma^{\prime}$ can be obtained with this approximation from a straightforward scalar tomography inversion of the Stokes- $I$ data. 


\section{Test simulations}

\subsection{The coordinate system}

The center of the main reference system is situated in the center of the sun. The $z$-axis is parallel to the sun's rotation axis and directed to the north pole. The $x$-axis is connected to some fixed point on the solar equator, and the $y$-axis is chosen in a way giving a righthanded reference system. The investigated volume is bounded by two spheres with radial distances $R_{\odot}$ and $R_{\text {out }}=2 R_{\odot}$. A rectangular discretization grid was chosen which has equidistant spacing and is aligned with the $x, y$ and $z$ coordinate axes (Fig. 6.1). The cells that cross the inner (the sun's surface) and outer spherical boundaries of the investigated volume are cut by these boundaries, i.e. they are not cubic. Within each cell of the grid, the magnetic field is assumed to be constant. The cells are numbered, and it is possible to create a column-matrix $\mathbf{X}$ the elements of which are the components of the magnetic field vector, $\boldsymbol{B}=B^{x} \hat{\boldsymbol{e}}_{\boldsymbol{x}}+B^{y} \hat{\boldsymbol{e}}_{\boldsymbol{y}}+B^{z} \hat{\boldsymbol{e}}_{\boldsymbol{z}}$, at the cell's center of gravity:

$$
X_{3 i-2}=B_{i}^{x}, \quad X_{3 i-1}=B_{i}^{y}, \quad X_{3 i}=B_{i}^{z} .
$$

\subsection{Discretization of the divergence operator}

To calculate the regularization term $F_{\text {reg }}$ in (3.13), the Gauss-Ostrogradskii theorem was used, which states that the flux of some vector field through a closed surface equals the integral of the divergence of the field over the volume bounded by this surface. So, as-
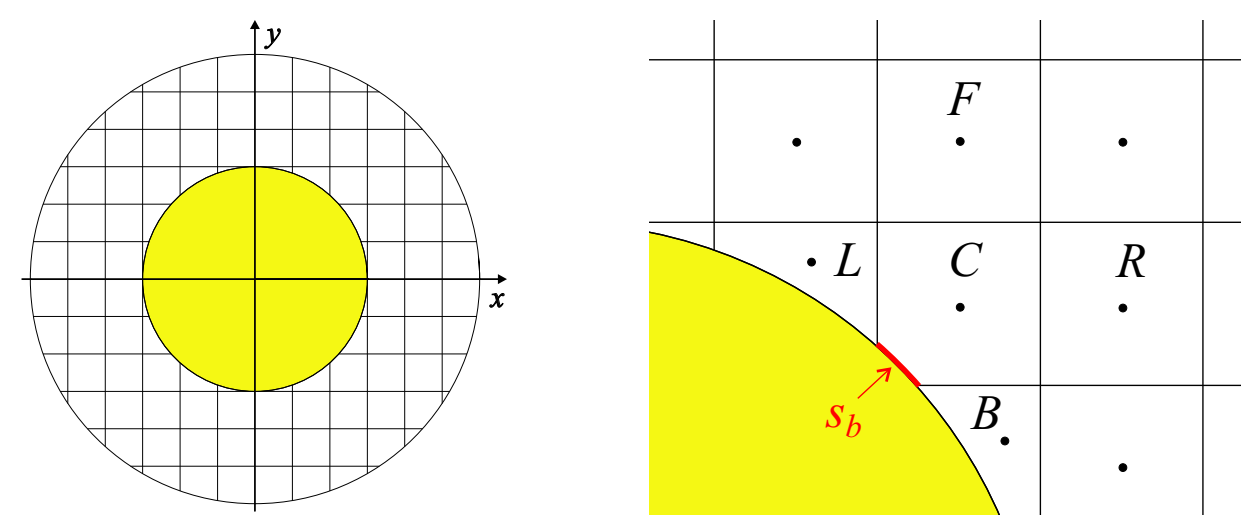

Figure 6.1: The example of $12 \times 12 \times 12$ grid: cross section by the plane $z=0$. Yellow area is the Sun. 
suming that $\nabla \cdot \boldsymbol{B}$ is constant within each cell $i$, one obtains

$$
\begin{array}{r}
\int_{\text {Corona }}(\nabla \cdot \boldsymbol{B})^{2} d V=\sum_{\text {All cells } i}\left[\int_{i \text {-th cell }}(\nabla \cdot \boldsymbol{B})^{2} d V\right]= \\
=\sum_{\text {All cells } i}\left[\left(\frac{\Phi_{i}}{V_{i}}\right)^{2} \int_{i \text {-th cell }} d V\right]=\sum_{\text {All cells } i} \frac{\Phi_{i}^{2}}{V_{i}}
\end{array}
$$

where $\Phi_{i}$ is the net magnetic flux out of the $i$-th cell, and $V_{i}$ is the volume of the $i$-th cell. The $V_{i}$ has the character of weighting coefficient, and it was dropped to simplify the calculations. Let us denote the $i$-th cell as " $\mathrm{C}$ "(central) and the surrounding cells as "L"(left), "R"(right), "F"(forward), "B"(backward), "U"(upper) and "'D"(down) as shown at Fig. 6.1b (upper and lower cells are not shown). The cell may be cut by the spherical boundary surface. The cut area is denoted by $S_{b}$. Then, a formula for the calculation of the net flux out of $i$-th cell used in simulations can be derived from the linearly interpolated field on each cell face, i.e.

$$
\begin{aligned}
\Phi_{i}=-\frac{B_{L}^{x}+B_{C}^{x}}{2} S_{L}+\frac{B_{R}^{x}+B_{C}^{x}}{2} & S_{R}+\frac{B_{F}^{y}+B_{C}^{y}}{2} S_{F}-\frac{B_{B}^{y}+B_{C}^{y}}{2} S_{B}+ \\
+ & \frac{B_{U}^{z}+B_{C}^{z}}{2} S_{U}-\frac{B_{D}^{z}+B_{C}^{z}}{2} S_{D}-\Phi_{b},
\end{aligned}
$$

where $S_{L}, S_{R}, S_{F}, S_{B}, S_{U}$, and $S_{D}$ are the areas of the faces common for the $i$-th cell and respective surrounding cells. If $i$-th cell intersects with the boundary surface, $\Phi_{b}$ is the flux out of the boundary area $S_{b}$ (see Fig. 6.1), otherwise $\Phi_{b}=0$. If $S_{b}$ lies on the solar surface, the representative field is directly taken from the surface boundary data. The flux through the outer spherical boundary is assumed to be unknown and, therefore, we do not include the flux through the cells cut by the outer boundary in the sum (6.2), but the magnetic field vectors defined at these cells are used to define the fluxes through the other cells. This makes the problem more ill-posed. For late practical calculations, especially when the outer boundary sphere exceeds $2 R_{\odot}$, the field on the outer sphere could be constrained to be radial. So, by defining the fluxes in this manner, we can write the regularization term in the matrix form (3.14).

In the simulations showed below it was used a rectangular grid of $40 \times 40 \times 40$ cells.

\subsection{Discretization of the line-of-sight integration}

Although every image pixel has a finite size, we approximate them by a point at the center of the physical pixel. So, the LOS volume from which the emission is projected onto the pixel is approximated by a line. This approximation is commonly used in many tomographic applications (Natterer 1986). It does not cause a big error in the case when the pixel size in the image is less than the cell size chosen for the grid. The distribution of the pixels in one image is set to be uniform with coordinates (figure 3.1):

$$
\begin{aligned}
& x_{p}=-R_{\text {out }}+\Delta x / 2+(i-1) \cdot \Delta x \\
& y_{p}=-R_{\text {out }}+\Delta y / 2+(j-1) \cdot \Delta y,
\end{aligned}
$$


where $i$ and $j$ are integer numbers, $\Delta x$ and $\Delta y$ are the cell sizes in $x$ and $y$ directions, respectively, and $R_{\odot}^{2}<x_{p}^{2}+y_{p}^{2}<R_{\text {out }}^{2}$.

The observations are assumed to be made from the Earth at a time when the Sun's rotation axis is perpendicular to the view direction $(\theta=\pi / 2$ in the Fig. 3.1), and due to the smallness of the variation of $\theta$ during one solar rotation, we assume that the angle is constant during one solar rotation. The angle $\phi$ varies in the simulations from 0 to $360^{\circ}$ in steps of $10^{\circ}$.

The minimizing function in the case of the longitudinal Zeeman effect data can be written in the matrix form (see eq. (3.12) and (3.14)) as follows:

$$
F=\mu \cdot F_{\text {tomo }}+F_{\text {reg }}=\mu \cdot|\mathbf{A} \cdot \mathbf{X}-\mathbf{Y}|^{2}+|\mathbf{G} \cdot \mathbf{X}-\mathbf{B}|^{2} .
$$

We have placed the regularization parameter at the tomographic term $F_{\text {tomo }}$ but not at the regularization term. This only affects the value of the resulting function $F$ but not it's behavior. The matrices here have a similar meaning as in (3.12) and (3.14). The components $X_{3 j-k}(k=2,1,0)$ of the column-vector $\mathbf{X}$ contains the components of the vector $\boldsymbol{B}$ in the $j$-cell, and $y_{i}$ is the data value for the $i$-th ray, where the index $i=1, \ldots, m$ counts the view angles, $\phi$ and $\theta$, and the pixel position $\left(x_{p}, y_{p}\right)$. The matrix element $a_{i, 3 j-k}$ represents the projection of the volume element $j$ along the LOS related to the pixel $i$, multiplied with some distance depended coefficient $k(r)$ and with the components of the unit vector along the LOS. The coefficient $k(r)$ is assumed to be constant within a cell. In the case of Faraday-effect data, the coefficient $k(r)$ is the electron density. In the case of Zeeman-effect data, the coefficient $k(r)$ is defined from the magnetograph formula (5.64), when the alignment factor, $\sigma_{0}^{2}$ (5.56), is set to zero. The radial dependence of $k(r)$ in this case does not principally differ from the radial dependence of the electron density. "Principally" means that their power exponents do not differ by more than one unit. Therefore, to show the possibility of the reconstruction method we assumed that the radial dependence of the coefficient $k(r)$ is the same like for the electron density defined in (5.92). Here, we assumed that the alignment factor is set to zero. Otherwise, the minimizing function cannot be expressed in the linear form (6.5), but must be derived by using the general form (5.64) of the magnetograph formula.

The number of the projections was set to 36 , and they were equispaced in the all range of angles from 0 to $2 \pi$.

\subsection{Magnetic field configuration for the test calculations}

The model field configuration is a dipole, with the dipole axis being inclined in the $x-z$ plane by $10^{\circ}$ with respect to the Sun's rotation axis ( $z$-axis). We added to the main dipole field a perturbation which is induced by a circular current in the $x$ - $z$-plane with radius $R_{\odot} / 2$ and center situated in the point $\left(R_{\odot} / 2 ; 0 ; 0\right)$ (see Fig. A.2). This perturbation adds a non-potential component to the test field which cannot be reconstructed by extrapolation methods from the surface data. The perturbation also modifies the axially symmetric unperturbed model to be a non-axially-symmetric model. The reconstruction of this perturbation with tomography data therefore represents a critical test of our method.

In the calculations below, we used arbitrary units for $\boldsymbol{B}$. At the point $\left(1.4 R_{\odot} ; 0 ; 0\right)$ close to the perturbing current, the field strength of the main dipole component in the arbitrary units is 3.5 , while the strength of the perturbing field component at this point, one cell spacing away from the perturbing current, is 2.8 . 


\subsection{Reconstruction based on the Zeeman-effect data}

From the model test field configuration the model data were calculated by a simple forward step. Random noise was added to the tomography data and surface magnetic field data at the photosphere (inner boundary condition) by multiplying the exact data and boundary conditions by $(1+\delta)$, where $\delta$ is a uniformly distributed random number in the range $\left(-\varepsilon_{\mathrm{n}} ;+\varepsilon_{\mathrm{n}}\right)$. The simulations were done with a noise level $\varepsilon_{\mathrm{n}}=0.05$, i.e. $5 \%$.

To minimize the function (6.5), the conjugate gradient method was used. The potential field reconstruction described in the section A.1 was used as initial field configuration for the iterations (Fig. A.3). The list of performed test reconstructions is shown in Table A.2. In the further discussions, we will use the notations in this table for the performed reconstructions.

Due to the very strong radial dependence of the electron density, the signal in a ray which passes the Sun at a greater distance is much less than for closer rays. Therefore, the contribution from these distant rays to the minimizing function is small. To increase the contribution of signals from distant rays, and to stabilize their numerical reconstruction, the data of pixel $\left(x_{p} ; y_{p}\right)$ was weighted with a function

$$
w_{s c}\left(r_{p}\right)=\frac{r_{p}^{4}}{n_{e}\left(r_{p}\right)}
$$

where $r_{p}=\sqrt{x_{p}^{2}+y_{p}^{2}}$.

During the minimization procedure, two questions arose: How to choose the value of the regularization parameter $\mu$, and What is the optimal number of iterations. These problems may be solved by using the L-curve method (Section 2.4). The Fig. 6.2 shows the evolution of the logarithm of the data error, $F_{\text {tomo }}$, versus the logarithm of the regularization term, $F_{\text {reg }}$, during the iterations for different values of $\mu$. A critical value for $F_{\text {tomo }}$ is the data noise level $\mathcal{E}_{\text {tomo, }}$, which in this example was calculated as

$$
\mathcal{E}_{\text {tomo }}=\left|\mathbf{Y}_{n}-\mathbf{Y}_{\mathbf{0}}\right|^{2}
$$

where $\mathbf{Y}_{n}$ and $\mathbf{Y}_{\mathbf{0}}$ are the tomography data with and without noise, respectively. In Fig. $6.2, \ln \mathcal{E}_{\text {tomo }}$ is shown as a horizontal dashed line. The difference in the (6.7) is just random noise with known distribution function which is added to the noiseless data $\mathbf{Y}_{\mathbf{0}}$. For real problems, $\mathbf{Y}_{\mathbf{0}}$ is not known, and $\mathcal{E}_{\text {tomo }}$ may be estimated from the instrument noise level. In the diagram $\ln F_{\text {tomo }}$ versus $\ln F_{\text {reg }}$, the iterations asymptotically converge to the Lcurve. Fig. 6.4 shows that the solutions obtained after 5000 iterations lie very close to the L-curve. The corner of the L-curve however lies much below the data noise level $\mathcal{E}_{\text {tomo }}$. In this case the value of $\mu$ found from the L-curve is not optimal (Hansen 1993; Hanke 1996).

All evolution lines in the Fig. 6.2 cross below the data noise level $\mathcal{E}_{\text {tomo }}$. However, when the error $F_{\text {tomo }}$ becomes smaller than $\mathcal{E}_{\text {tomo }}$, we begin to fit our reconstruction to the noise in the data. For ill-conditioned problems, this results in a strongly amplified noise in the reconstruction model. We therefore stop the iteration when $F_{\text {tomo }} \cong \mathcal{E}_{\text {tomo }}$. So, this criterion (Mozorov's criterion) determines the number of iterations in our case to be approximately 47 for values of $\mu$ in the range from $10^{-5}$ to $10^{5}$. But the problem of choosing of the optimal value of $\mu$ is still open. 


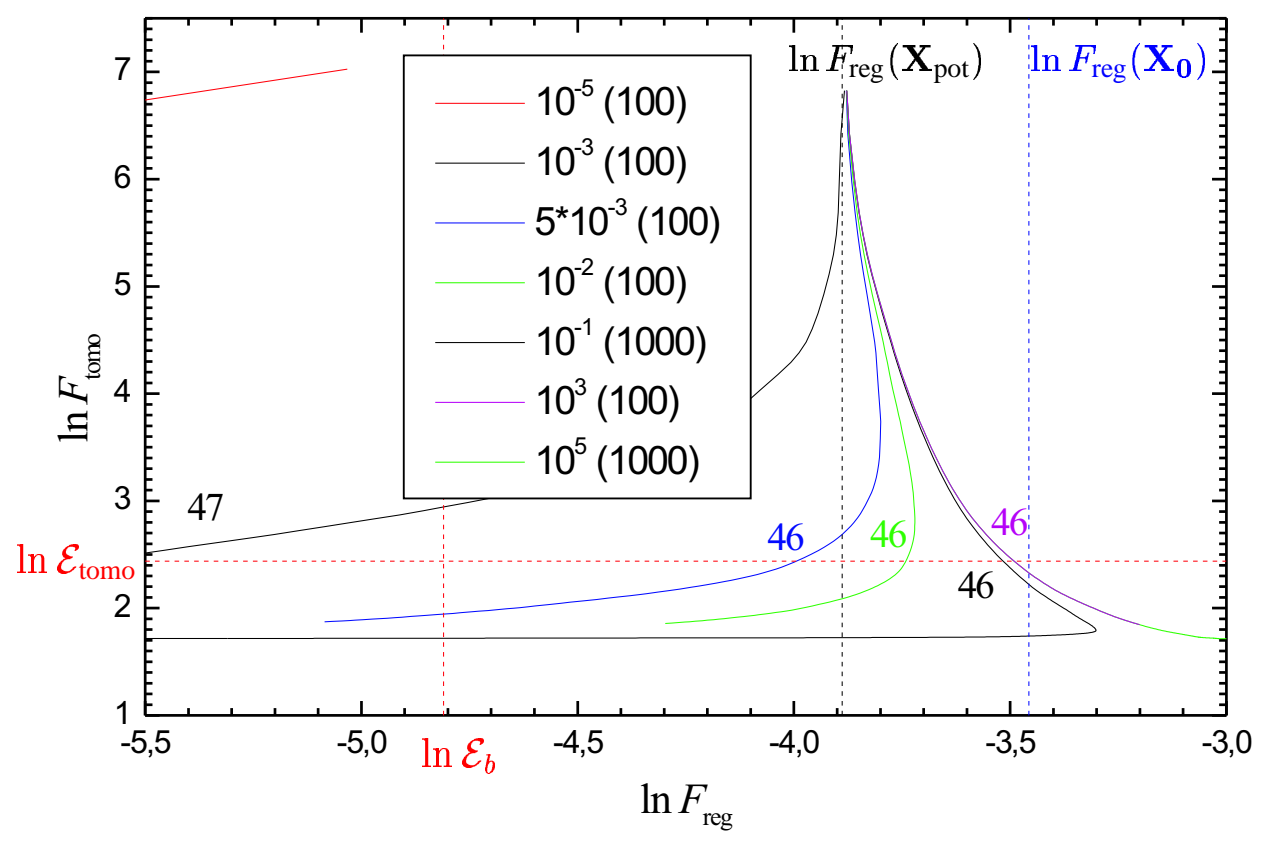

Figure 6.2: The evolution of the L-curve during the iterations for different values of $\mu$. Colored lines correspond for the values of $\mu$ listed in the insert. The number of iterations which were done to plot the lines are pointed out in the brackets beside. Colored numbers near the lines are the number of iterations which were done to reach the data noise level $\mathcal{E}_{\text {tomo }}$ (red dashed horizontal line).

The iteration results for different $\mu$ mainly differ in the final value of the regularization term $F_{\text {reg }}$. A critical number for $F_{\text {reg }}$ is the logarithm of the regularization term, $F_{\text {reg }}\left(\mathbf{X}_{\mathbf{0}}\right)$, for the original field $\mathbf{X}_{\mathbf{0}}$. Theoretically, the value of $F_{\text {reg }}\left(\mathbf{X}_{\mathbf{0}}\right)$ must be zero. But due to the noise in the boundary data, and the error in the model space due to discretization, this value is greater than zero. Hence, $F_{\text {reg }}\left(\mathbf{X}_{\mathbf{0}}\right)$ is an estimate of the discretization error and noise in the boundary magnetic field. Its value in our example is indicated by a blue dashed vertical line in Fig. 6.2. The boundary noise level is denoted through $\mathcal{E}_{b}$.

Unfortunately, for a real problem, $\mathbf{X}_{\mathbf{0}}$ is unknown and we can only roughly estimate the discretization error in the regularization term by applying it to a potential field approximation $\mathbf{X}_{\text {pot }}$. The black vertical line in Fig. 6.2 corresponds to the logarithm of the regularization term, $F_{\text {reg }}\left(\mathbf{X}_{\text {pot }}\right)$, for the potential field approximation $\mathbf{X}_{\text {pot }}$ which lacks the current perturbation. Here $\ln F_{\text {reg }}\left(\mathbf{X}_{\text {pot }}\right)<\ln F_{\text {reg }}\left(\mathbf{X}_{\mathbf{0}}\right)$, because the relatively large field in the perturbed area causes a larger discretization error (the value of the error depends on the field-strength gradient).

Another way to estimate the lower value of the $F_{\text {reg }}$ is the following. The elements of the matrix $\mathbf{G} \cdot \mathbf{X}_{\mathbf{0}}$ (see eq. (6.5)) are the net flux through corresponding cells. The same elements of the matrix $\mathbf{B}$ for which the corresponding cells are not cut by the boundaries are strictly zero. The elements for which the corresponding cells are cut by the boundaries are calculated from the observed on the boundaries magnetic field. They usually are not zero and under influence of noise.

It is possible to divide the regularization term into two parts: the first is calculated by involving only the cells which are not cut by the spherical boundaries, $F_{\text {reg }}^{(\mathrm{n})}$, and the 


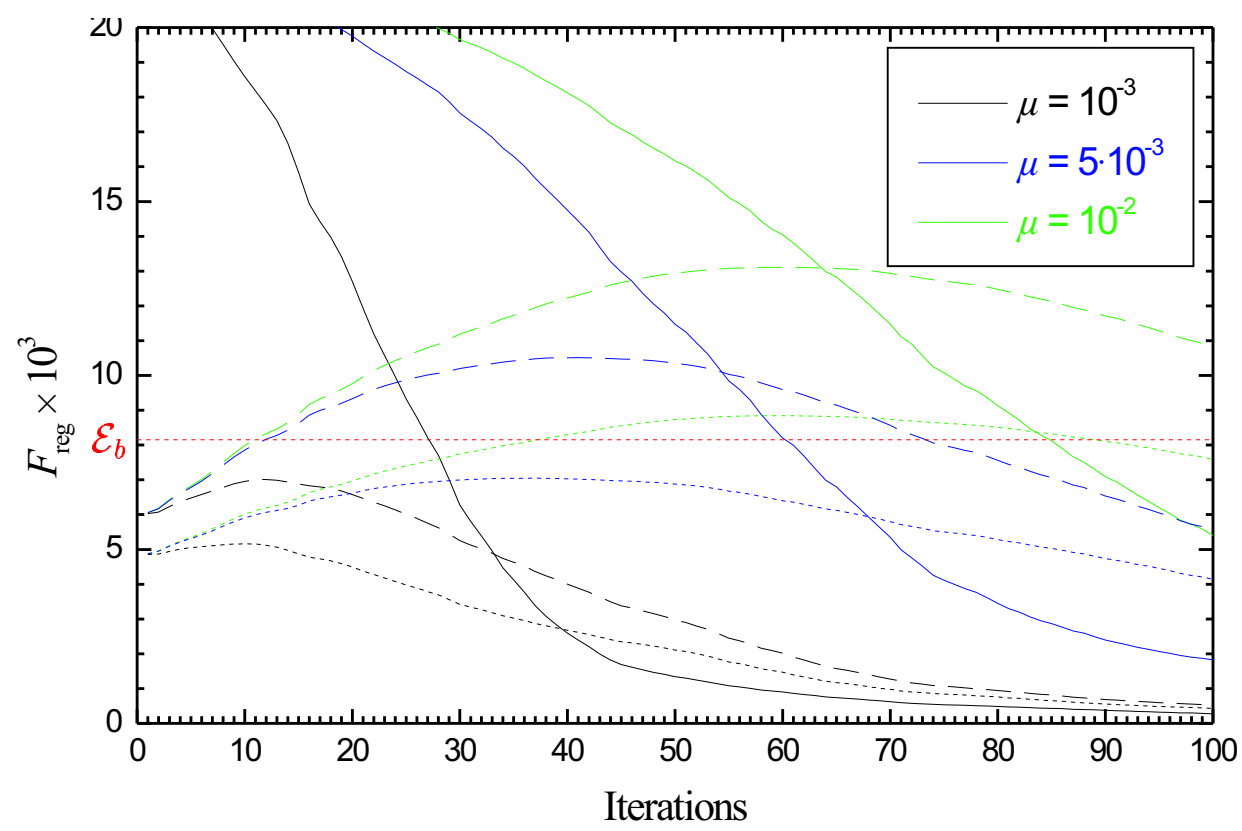

Figure 6.3: The regularization term calculated separately for the cells which are cut (solid lines) and not cut (long dashed lines) by the spherical boundaries. The short dashed lines represent $F_{\text {reg }}$ calculated for the uncut cells, but without considering the cells in the perturbed area.

second involves only the cells which are cut by these boundaries, $F_{\text {reg }}^{(\mathrm{b})}$ (the symbols "n" and "b" are just notations here, not an exponents). The discretization error is mainly introduced by the $F_{\text {reg }}^{(\mathrm{n})}$ because the number of cells involved in the calculation of $F_{\text {reg }}^{(\mathrm{n})}$ is much higher than for $F_{\text {reg }}^{(\text {b) }}$. In the case of using a high-order interpolation in the calculation of the regularization term the discretization error can be neglected, and even for the rough linear interpolation used here this error is not significant. Although $F_{\text {reg }}^{(\mathrm{n})}$ can be compatible with $F_{\text {reg }}^{(b)}$ due to the much larger amount of cells involved in the calculation of the $F_{\text {reg }}^{(\mathrm{n})}$, the error contribution from the noisy boundary data is higher in the presented example. Fig. 6.3 shows the values of $F_{\text {reg }}$ calculated separately for these two kinds of cells during the iterations for several values of $\mu$. Here we see that after 47 iterations $F_{\text {reg }}^{(\mathrm{b})}=\mathcal{E}_{b}$ when $\mu \approx 5 \cdot 10^{-3}$. Even if we take $\mu=10^{-3}$, it would not be a big fit to the noise in the boundary data. The choice of the value of $\mu$ depends on which regions must be reconstructed better than others.

It should be noted that the regularization term represented by the condition $\nabla \cdot \boldsymbol{B}=0$ has a large null-space. Therefore, a field for which $F_{\text {reg }}=0$ is not obligatory smooth. The regularization term $F_{\text {reg }}$ differs here from conventionally chosen regularization operators (Section (2.4)), which are often proper smoothing operators. Let us introduce a 


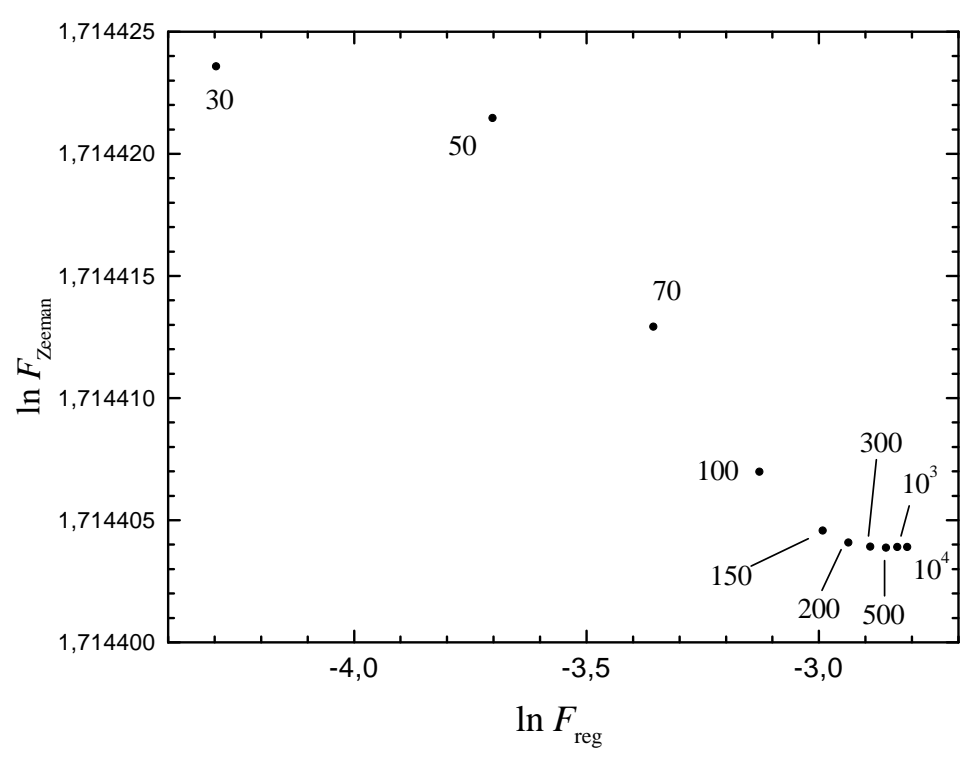

Figure 6.4: The L-curve for the reconstruction based on the Zeeman-effect data with $5 \%$ noise level. The numbers connected with the points indicate values of $\mu_{\text {reg. }}$.

smoothness operator as

$$
\begin{aligned}
& F_{\mathrm{sm}}=\sum_{i, j, k} {\left[\left(B_{i, j, k}^{x}-B_{i+1, j, k}^{x}\right)^{2}+\left(B_{i, j, k}^{x}-B_{i, j+1, k}^{x}\right)^{2}+\left(B_{i, j, k}^{x}-B_{i, j, k+1}^{x}\right)^{2}+\right.} \\
&+\left(B_{i, j, k}^{y}-B_{i+1, j, k}^{y}\right)^{2}+\left(B_{i, j, k}^{y}-B_{i, j+1, k}^{y}\right)^{2}+\left(B_{i, j, k}^{y}-B_{i, j, k+1}^{y}\right)^{2}+ \\
&\left.+\left(B_{i, j, k}^{z}-B_{i+1, j, k}^{z}\right)^{2}+\left(B_{i, j, k}^{z}-B_{i, j+1, k}^{z}\right)^{2}+\left(B_{i, j, k}^{z}-B_{i, j, k+1}^{z}\right)^{2}\right]
\end{aligned}
$$

where $B_{i, j, k}^{x}, B_{i, j, k}^{y}, B_{i, j, k}^{z}$ are the $x$-, $y$ - and $z$-components of the magnetic field vector at the cell with indexes $i, j, k$ which are counted along the $x$-, $y$ - and $z$-axes, respectively. The smoothness term was not involved in the inversion procedure, but it is useful to plot the behavior of the logarithm of the data's error versus the logarithm of the smoothness term, $F_{\mathrm{sm}}$, during the iterations for the different values of $\mu$ (Fig. 6.5). Fig. 6.5 shows that as the iteration proceeds and $F_{\text {tomo }}$ decreases the magnetic field model involves more and more structures and $F_{\mathrm{sm}}$ increases. The blue dashed vertical line there corresponds to the value of the smoothness term for the original field $F_{\mathrm{sm}}\left(\mathbf{X}_{\mathbf{0}}\right)$ and can serve as a reference of values for $F_{\mathrm{sm}}$ we should achieve with our solution. However, we also do not want to smooth solutions for which $F_{\mathrm{sm}}$ is less than $F_{\mathrm{sm}}\left(\mathbf{X}_{\mathbf{0}}\right)$, because we would loose real features in the reconstruction. Fig. 6.5 shows that the values of $\mu$ laying between $10^{-3}<\mu<5 \cdot 10^{-3}$ satisfy these criteria. For $\mu<10^{-3}$ we obtain results which show more structures (larger $F_{\mathrm{sm}}$ ) but also include more noise. The reconstruction for $\mu=10^{-3}$ after 47 iterations is shown in Fig. A.4. We see that it is a better reconstruction than for the potential field approximation, at least it is possible to see the perturbed region.

Similar to the analysis described above, we have performed an analysis with $F_{\text {tomo }}$ replaced by $F_{\text {tomo }}^{\prime}$ which includes the data error only for those rays that pass through the region magnetically influenced by the current perturbation (perturbed region). In the 


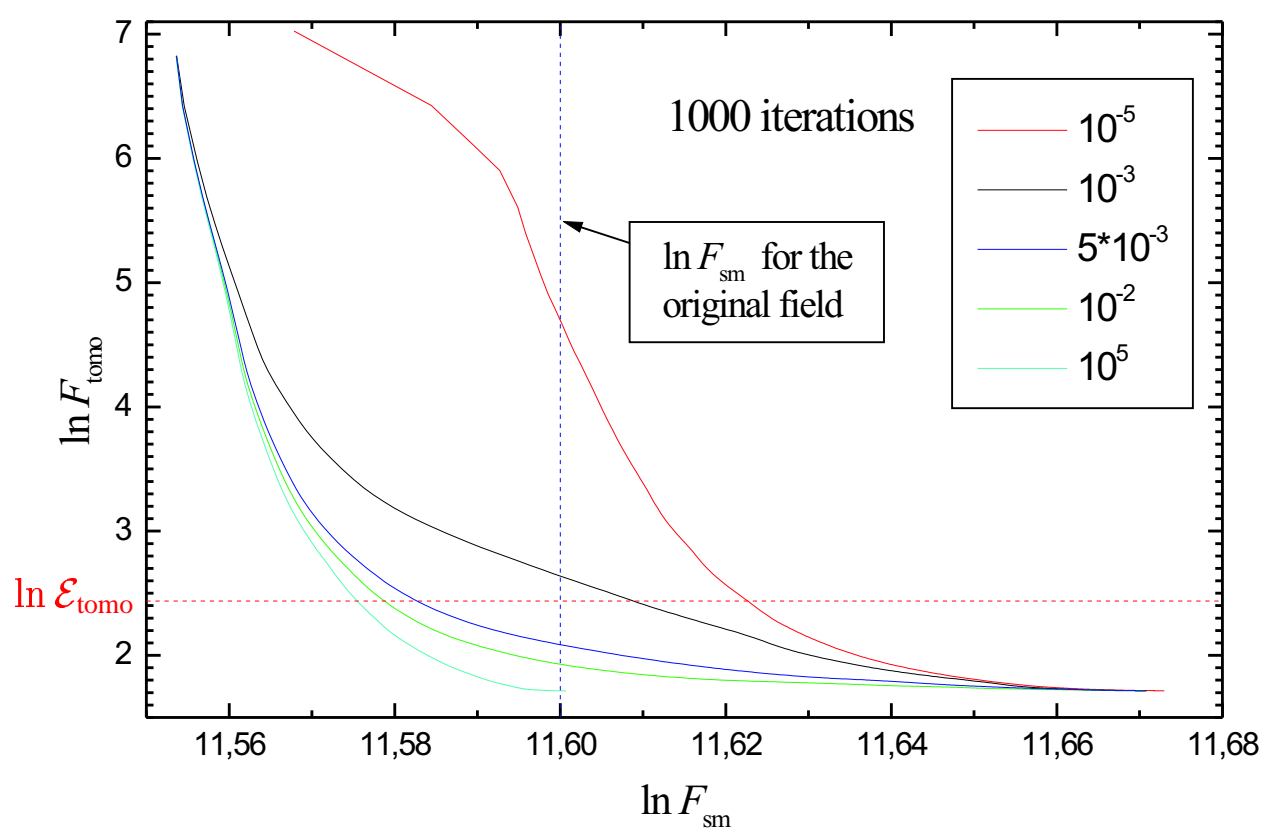

Figure 6.5: The behavior of the data's error versus the smoothness during the iterations for the different values of $\mu$. Colored lines correspond to the values of $\mu$ listed in the insert.

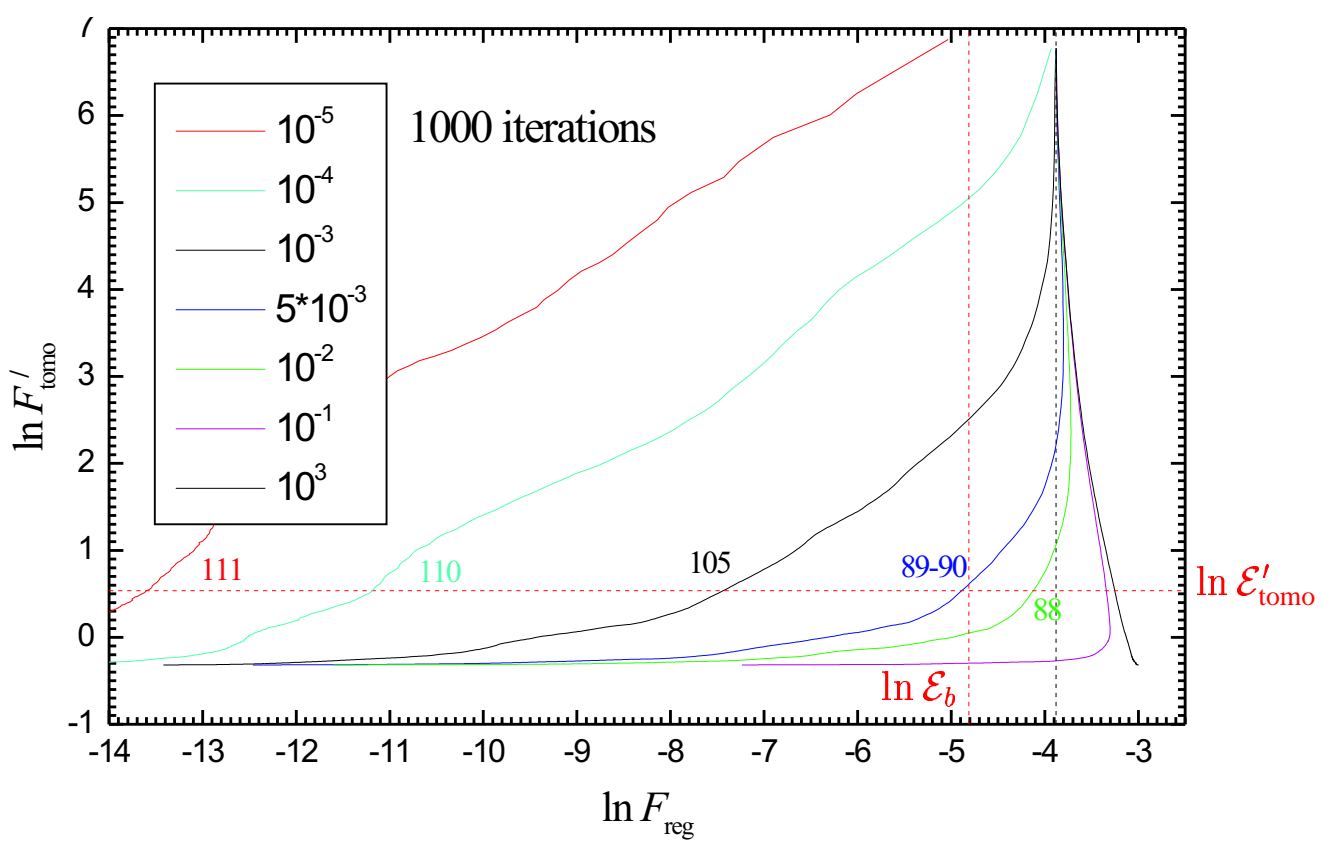

Figure 6.6: The evolution of the L-curve for the perturbed region during the iterations for different values of $\mu$. The colored lines correspond to the values of $\mu$ listed in the insert. The colored numbers near the lines are the number of iterations which were done to reach the data noise level $\mathcal{E}_{\text {tomo }}^{\prime}$.

following analyses, we restrict the analyzing part of the perturbed region by the planes $z= \pm R_{\odot} / 2, y / x=\tan \left( \pm \frac{\pi}{18}\right)$ and by the conditions $x>0, R_{\odot}^{2}<x^{2}+y^{2}+z^{2}<\left(2 R_{\odot}\right)^{2}$. 
The Fig. 6.6 shows the behavior of the logarithm of the $F_{\text {tomo }}^{\prime}$ versus the logarithm of the regularization term, $F_{\text {reg }}$, calculated for all the cells, during the iterations for the different values of $\mu$. Analogously with the Fig. 6.2, we introduce the data noise level $\mathcal{E}_{\text {tomo }}^{\prime}$ for the rays passing through the perturbed region. Because the noise depends on the magnitude of the data, the value of $\mathcal{E}_{\text {tomo }}^{\prime}$ is not simply proportional to the number of rays that passed through the chosen region. The value of the $\mathcal{E}_{\text {tomo }}^{\prime}$ is marked by a red dashed horizontal line in the Fig. 6.6. The boundary data noise level, $\mathcal{E}_{b}$, is not so critical here because now we are interested mainly in the perturbed region. To reach the noise level $\mathcal{E}_{\text {tomo, }}^{\prime}$, it is nessecary to perform 105 iterations when $\mu=10^{-3}$. Figures A.5 shows the reconstruction for $\mu=10^{-3}$ after 99 iterations.

\subsection{Reconstruction based on the Hanle-effect data}

For the reconstruction based on the Hanle-effect measurements of the Stokes- $I,-Q$ and- $U$ components we minimize the function

$$
\begin{gathered}
F=F_{\text {reg }}+\mu_{I} F_{\text {tomoI } I}+\mu_{Q} F_{\text {tomo } Q}+\mu_{U} F_{\text {tomoU }}=|\mathbf{G} \cdot \mathbf{X}-\mathbf{B}|^{2}+ \\
+\sum_{i}\left[\mu_{I}\left(I_{i}^{\mathrm{s}}(\mathbf{X})-I_{i}^{\mathrm{d}}\right)^{2}+\mu_{Q}\left(Q_{i}^{\mathrm{s}}(\mathbf{X})-Q_{i}^{\mathrm{d}}\right)^{2}+\mu_{U}\left(U_{i}^{\mathrm{s}}(\mathbf{X})-U_{i}^{\mathrm{d}}\right)^{2}\right]
\end{gathered}
$$

Here, the matrix $\mathbf{G}$ and column-matrices $\mathbf{X}$ and $\mathbf{B}$ are the same as in (6.5), $I_{i}^{\mathrm{d}}, Q_{i}^{\mathrm{d}}$ and $U_{i}^{\mathrm{d}}$ are the data of the $i$-th ray for the Stokes- $I,-Q$ and $-U$ components, respectively, and $I_{i}^{\mathrm{s}}$, $Q_{i}^{\mathrm{s}}$ and $U_{i}^{\mathrm{s}}$ are the simulated Stokes- $I,-Q$ and $-U$ components, respectively, for the $i$-th ray, i.e.,

$$
I_{i}^{\mathrm{s}}=\int_{i \text {-th LOS }} \varepsilon_{I} d l, \quad Q_{i}^{\mathrm{s}}=\int_{i \text {-th LOS }} \varepsilon_{Q} d l, \quad U_{i}^{\mathrm{s}}=\int_{i \text {-th LOS }} \varepsilon_{U} d l,
$$

where $\varepsilon_{I}, \varepsilon_{Q}$ and $\varepsilon_{U}$ are the magnetic-field-dependent Stokes- $I,-Q$ and $-U$ emission coefficients, respectively, that were calculated by (5.71) for the $10747 \AA$ line or by (5.86) for the $5303 \AA$ line.

Using the model field (Section 6.4) the test data were again calculated by a simple forward step. Then random noise was added to the tomography data and to the surface magnetic field data at the photosphere (inner boundary condition) by multiplying the test data and boundary conditions with $(1+\delta)$, where $\delta$ is a uniformly distributed random number in the range $\left(-\varepsilon_{\mathrm{n}} ;+\varepsilon_{\mathrm{n}}\right)$. The simulations were made, like for the Zeeman-effect case, with a noise level $\varepsilon_{\mathrm{n}}=0.05$, i.e. $5 \%$.

To minimize the function (6.9), the conjugate gradient method was used again. Only the Stokes- $Q$ and $-U$ signals were used in the minimization procedure (i.e. $\mu_{I}=0$ ), and we set $\mu_{Q}=\mu_{U}=\mu$ as their (Stokes- $Q$ and $-U$ ) dimensions are the same. Again, like for the Zeeman-effect case, the potential field approximation was used as initial field configuration for the iterations.

Similar to the Zeeman-effect reconstruction procedure, to increase contribution of signals from distant rays the data of pixel $\left(x_{p} ; y_{p}\right)$ was weighted with a function

$$
w_{s c}\left(r_{p}\right)=\frac{r_{p}^{2}}{n_{e}\left(r_{p}\right)}
$$




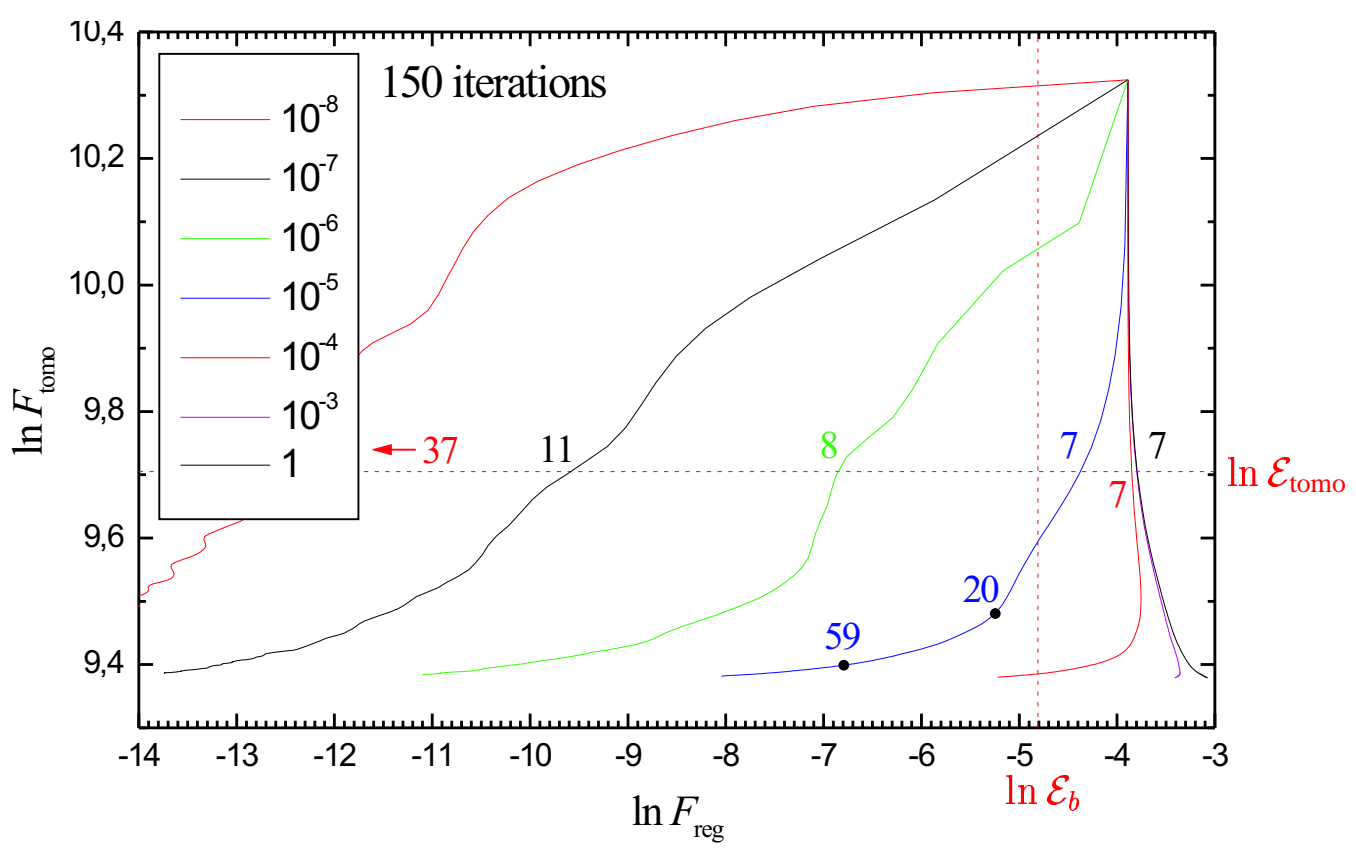

Figure 6.7: The evolution of the logarithm of the data error $F_{\text {tomo }}$ versus the logarithm of the regularization term $F_{\text {reg }}$ during the iterations for different values of $\mu$ and for the reconstruction based on the Hanle-effect data. The colored curves correspond to the values of $\mu$ listed in the insert. The colored numbers near the lines are the number of iterations which were needed to reach the data noise level $\mathcal{E}_{\text {tomo }}$ (red dashed horizontal line). $\mathcal{E}_{b}$ is the boundary noise level in the regularization term.

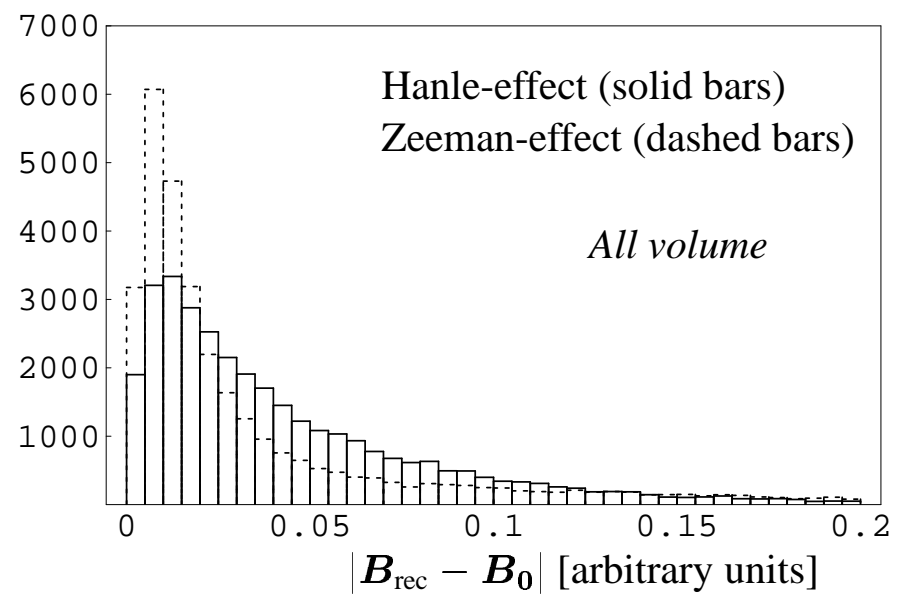

Figure 6.8: The histograms of the distribution of the difference vector magnitudes over the whole volume for the reconstructions H1 (Hanle-effect data, $\mu=10^{-5}, 7$ iterations, solid bars) and Z1 (Zeeman-effect data, $\mu=10^{-3}, 47$ iterations, dashed bars) 
where $r_{p}=\sqrt{x_{p}^{2}+y_{p}^{2}}$, and $n_{e}$ is electron density.

The Fig. 6.7 shows the behavior of the logarithm of the data error, $F_{\text {tomo }}$, versus the logarithm of the regularization term, $F_{\text {reg }}$, during the iterations for different values of $\mu$. The horizontal dashed line is the data noise level $\mathcal{E}_{\text {tomo }}$ calculated analogously to the case of the Zeeman-effect (eq.(6.7)), and $\mathcal{E}_{\mathrm{b}}$ marks the boundary data noise level in the regularization term. In order to obtain a tomography's data error $F_{\text {tomo }} \sim \mathcal{E}_{\text {tomo }}$ and a regularization error $F_{\text {reg }} \sim \mathcal{E}_{\mathrm{b}}$, like for the case of Zeeman-effect data, we chose $\mu=10^{-5}$ and stopped after 7 iterations (see Figs. A.6a,b,c for an overview of cross sections of the reconstructed field). To quantify the reconstruction error we calculated for every cell the magnitude of the difference vector between the reconstructed and the original model magnetic field vectors. For an ideal solution, these vectors must approach zero as close as possible. The histograms of the distribution of the magnitudes of the difference vectors for the three reconstruction methods are shown in Fig. 6.8. The distribution has a peak at the value of $\left|\boldsymbol{B}_{\text {rec }}-\boldsymbol{B}_{\mathbf{0}}\right|$ equal to $\sim 0.015$. Only in a very few cases exceeds the distance 0.2 , while the magnitudes of model field vectors, $\left|\boldsymbol{B}_{\mathbf{0}}\right|$, are distributed in the region $1<\left|\boldsymbol{B}_{\mathbf{0}}\right|<13.4$, with a maximum at $\sim 1.5$ and average value of $\approx 3.3$. So, the reconstruction error is relatively small.

To find the iteration number when we will have a better reconstruction for the perturbed area alone, we calculate the data noise level $\mathcal{E}_{\text {tomo }}^{\prime}$ only for the rays passing through the perturbed region like in the previous section. The data error $F_{\text {tomo }}^{\prime}$ calculated only for these rays reaches the level $\ln \mathcal{E}_{\text {tomo }}^{\prime}$ after $\sim 59$ iterations. Trying to keep the regularization term error the same like for the case of the Zeeman-effect data (i.e., near $\mathcal{E}_{\mathrm{b}}$ ), we have to stop the minimization procedure after 20-th iteration (see Figs. A.7a,b,c for an overview of cross sections of the reconstructed field).

\subsection{Comparison of the Hanle- and Zeeman-effect solu- tions}

Here, we will compare the solutions $\mathrm{H} 2$ based on the Hanle-effect data (with $\mu=10^{-5}$ and stopped after 20 iterations, Fig. A.7) and Z2 described in Section 6.5 (Zeeman-effect data, $\mu=10^{-3}, 99$ iterations). The histograms of the distribution of the magnitudes of the difference vectors in the perturbed region for these solutions are shown in the Fig. 6.9. The distributions have their maxima at values of $\left|\boldsymbol{B}_{\text {rec }}-\boldsymbol{B}_{\mathbf{0}}\right|$ equal to $\sim 0.1$ for the Hanle-effect reconstruction and $\sim 0.3$ for the Zeeman-effect reconstruction, and in only a few cases this value is near or greater 4 , while the magnitudes of the model field vector, $\left|\boldsymbol{B}_{\mathbf{0}}\right|$, in the perturbed region are distributed in the interval $1<\left|\boldsymbol{B}_{\mathbf{0}}\right|<13$.4, with maximum at $\sim 1.6$ and average value of $\approx 3.9$. Although, the bar near zero is for the Hanle-effect solution higher than for the Zeeman-effect solution, but the bars being more far from zero and ranging between the values $1<\left|\boldsymbol{B}_{\text {rec }}-\boldsymbol{B}_{\mathbf{0}}\right| \lesssim 2$ are higher than for the Zeeman-effect solution. Therefore, it is difficult to say which solution is better.

The Zeeman effect is insensitive to the irrotational part of the vector field, and in the present observing geometry when observation directions are all perpendicular to the rotation axis is insensitive to the $z$-component of the field vectors. Therefore, one would expect that a reconstruction based on Zeeman effect data gives worse result for these field components. The histograms in the Fig. 6.10 illustrate this situation. The his- 


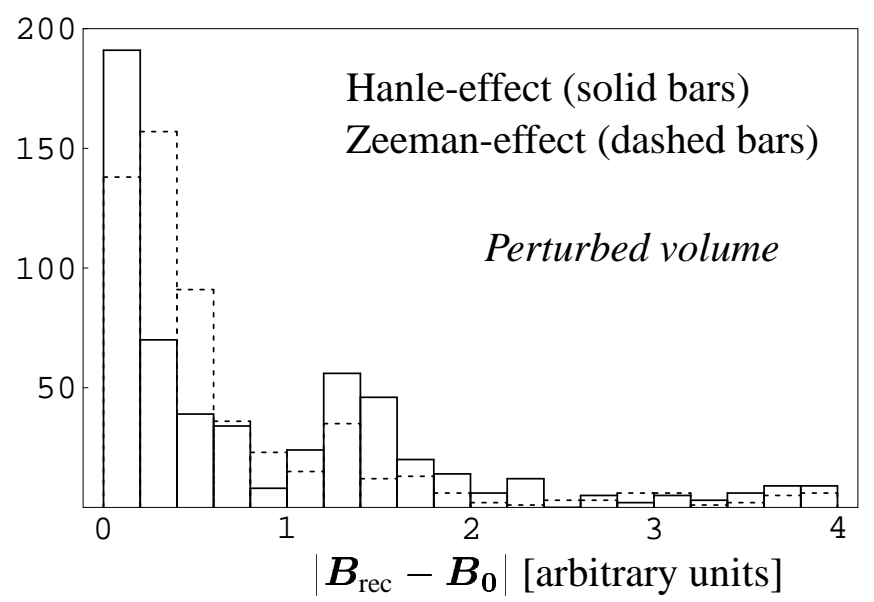

Figure 6.9: The histograms of the distribution of the difference vector magnitudes over the perturbed volume for the reconstructions $\mathrm{H} 2$ (Hanle-effect data, $\mu=10^{-5}, 20$ iterations, solid bars) and Z2 (Zeeman-effect data, $\mu=10^{-3}, 99$ iterations, dashed bars).

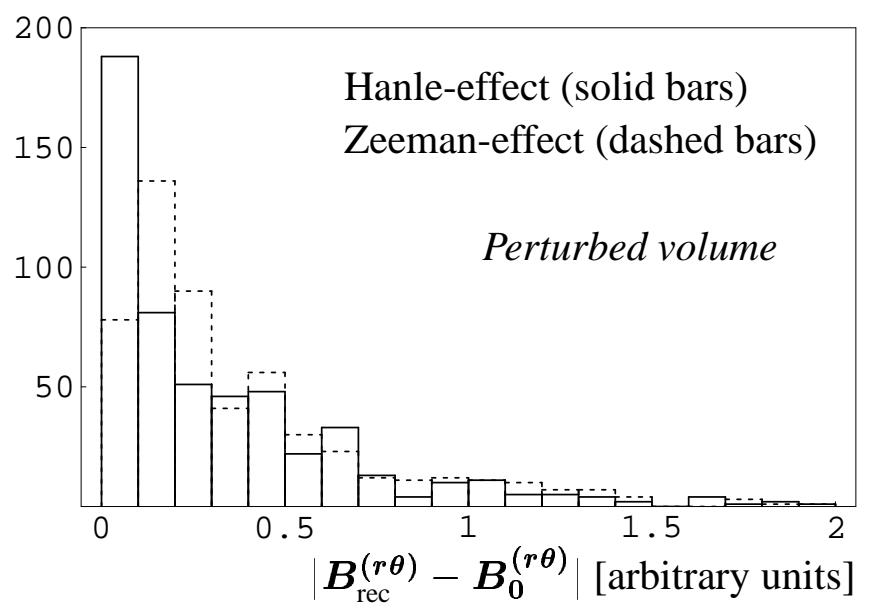

Figure 6.10: The histograms of the distribution of the errers of the meridional-plane component of the field vectors over the perturbed volume for the reconstructions H2 (Hanleeffect data, $\mu=10^{-5}, 20$ iterations, solid bars) and Z2 (Zeeman-effect data, $\mu=10^{-3}$, 99 iterations, dashed bars).

tograms shows the distributions of the errors of the reconstructed field vector components, $\left|\boldsymbol{B}_{\mathrm{rec}}^{(\boldsymbol{r} \boldsymbol{\theta})}-\boldsymbol{B}_{\mathbf{0}}^{(\boldsymbol{r} \boldsymbol{\theta})}\right|$, laying in the meridional planes defined by the rotation axis and point where field vector is looking for. Here, $\boldsymbol{B}^{(\boldsymbol{r} \boldsymbol{\theta})}=B^{(r)} \hat{\boldsymbol{e}_{\boldsymbol{r}}}+B^{(\theta)} \hat{\boldsymbol{e}_{\boldsymbol{\theta}}}$, where $B^{(r)}$ and $B^{(\theta)}$ are the radial and meridional components of the magnetic field vector, respectively. The $\hat{e_{r}}$ and $\hat{e_{\theta}}$ are the unit vectors defining the spherical coordinate system centered at the given point in the corona, $\hat{e_{r}}$ is co-directed with the radius vector, $\hat{e_{\theta}} \perp \hat{e_{r}}$, the vectors $\hat{\boldsymbol{e}_{\boldsymbol{r}}}, \hat{\boldsymbol{e}_{\boldsymbol{\theta}}}$ and $\boldsymbol{O} \boldsymbol{z}$ lie in the same plane, and $\hat{\boldsymbol{e}_{\boldsymbol{\theta}}} \cdot \boldsymbol{O} \boldsymbol{z} \geq 0$.

The Fig. $6.11 a$ shows the histogram of the distribution of the errors of the azimutal component $B^{(\phi)}$ of the reconstructed field vectors. We see that for this component the reconstruction based on the Zeeman-effect data gives better result than the one based on the Hanle-effect data. This is again understandable because, with the present viewing 

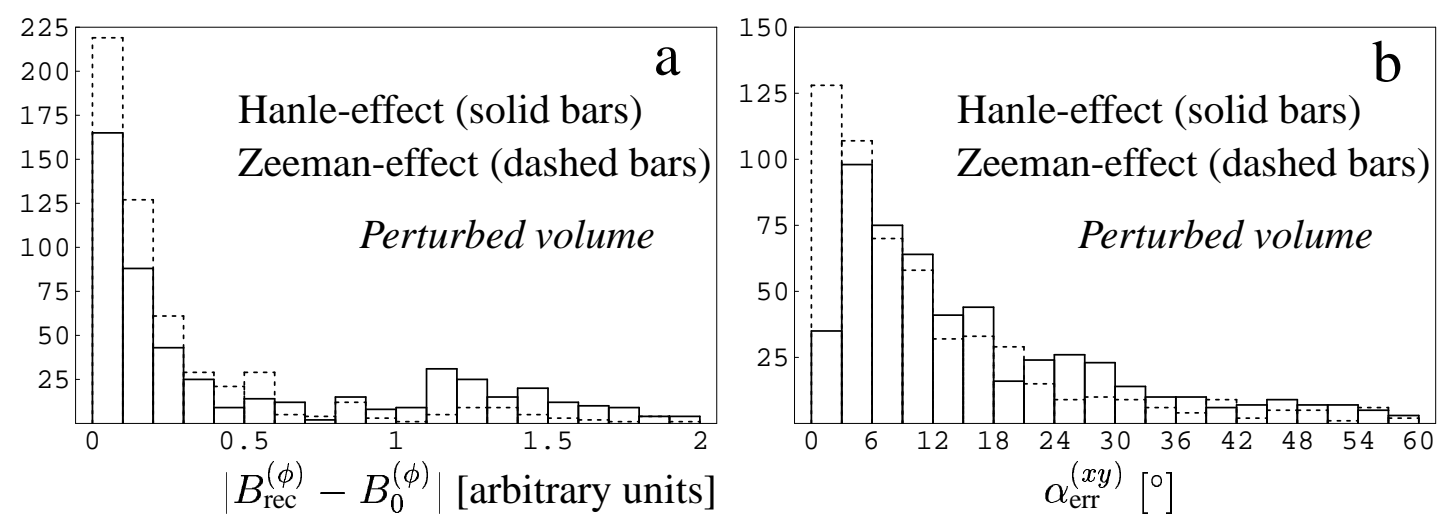

Figure 6.11: The histograms of the distributions of the errors of the azimutal components $B^{(\phi)}$ of the field vectors (a) and the angle between the $x$ - $y$-plane vector components of the reconstructed and model fields (b) over the perturbed volume for the reconstructions H2 (Hanle-effect data, $\mu=10^{-5}, 20$ iterations, solid bars) and Z2 (Zeeman-effect data, $\mu=10^{-3}, 99$ iterations, dashed bars)
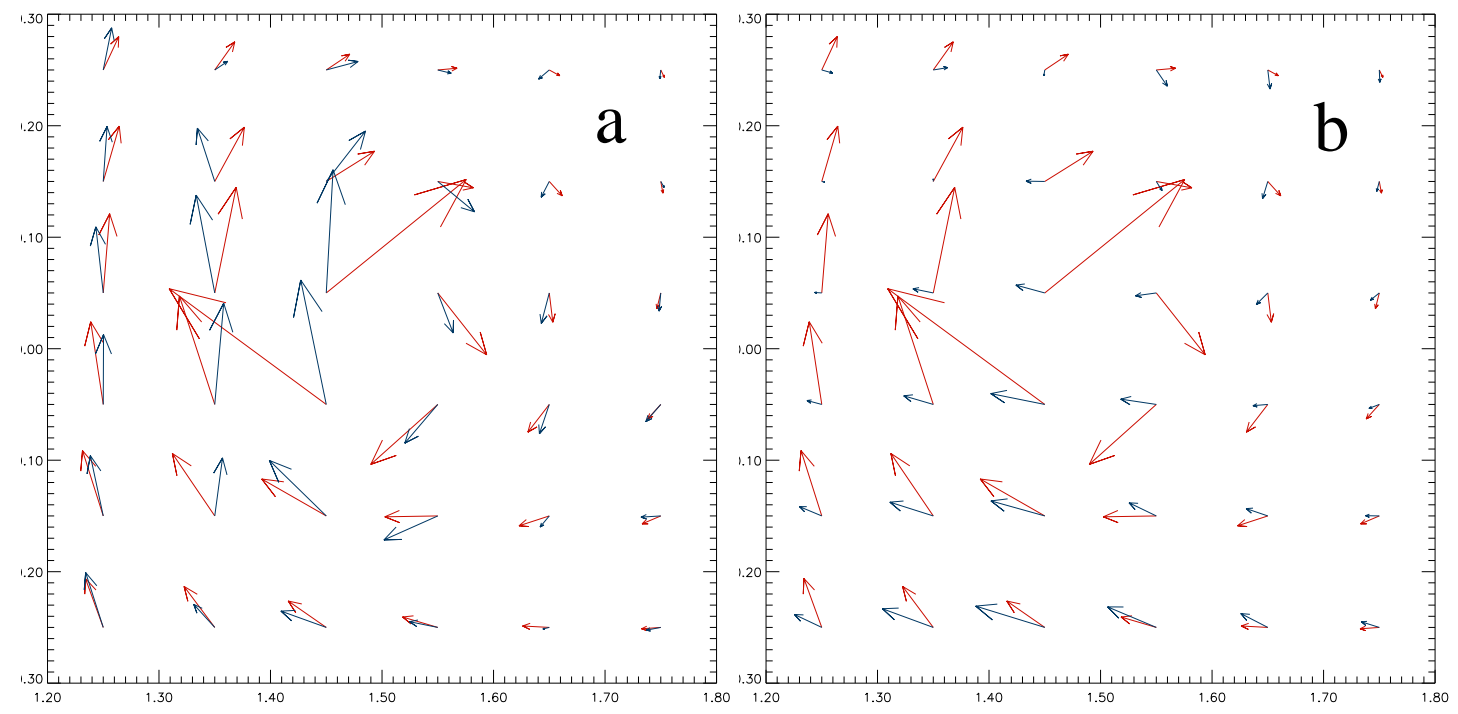

Figure 6.12: The cross-sections at the $z=0.15 R_{\odot}$ of the model field (red vectors) and reconstructed field (blue vectors). The reconstructions are Z2 based on the Zeeman-effect data (a) and $\mathrm{H} 2$ based on the Hanle-effect data (b)

geometry, the problem of definition of the azimutal field component is more ill-posed for the Hanle effect than for the Zeeman effect. Let us explain this in more detail. Only the $x$ - and $y$-components of the field vector contribute to the $B^{(\phi)}$, and the $x$-y-plane components of the field vectors give no contribution to the Stokes- $U$ signal. For the $x$ - $y$-plane components we only have information about the angle $\theta_{x y}$ between LOS and this vector component through the function $\sin ^{2} \theta_{x y}$ which introduces ambiguities, i.e. we have 4 possible directions of the field vector, and therefore the Hanle effect cannot distinguish a field vector directed along the LOS from one directed against the LOS, while the Zeeman effect does (due to the $\cos \theta_{x y}$ dependence). Moreover, the Hanle effect 
does not provide any information about the strength of the magnetic field.

Is it possible that the big error in the $B^{(\phi)}$ component in the perturbed region is due to the big error in the absolute value of the $x-y$-plane vector component but not due to its direction in these planes? Fig. 6.11b shows histogram of distribution of the angles between the $x-y$-plane vector component of the reconstructed and model fields. We see that this is not the case.

Finally, the Fig. 6.12 qualitatively shows the difference between the reconstructions and the original model field in the plane $z=0.15 R_{\odot}$ and around the perturbed region.

\subsection{Reconstruction based on the Hanle-effect: Zeeman- effect solution as initial field}

Even though the Zeeman-effect data give a better reconstruction for the particular field configuration than the Hanle-effect data, we want to explore in this section whether the reconstruction based on the Zeeman-effect data can be improved if they are supplemented by the Hanle-effect observations. For this purpose, we repeat the inversion calculations for the Hanle-effect data as in Section 6.6, but this time we use the Zeeman-effect solution $\mathrm{Z} 1$ (the solution with $\mu=10^{-3}$ obtained after 47 iterations) as initial field configuration in the iterations. All others parameters used in the iterations are the same as in section

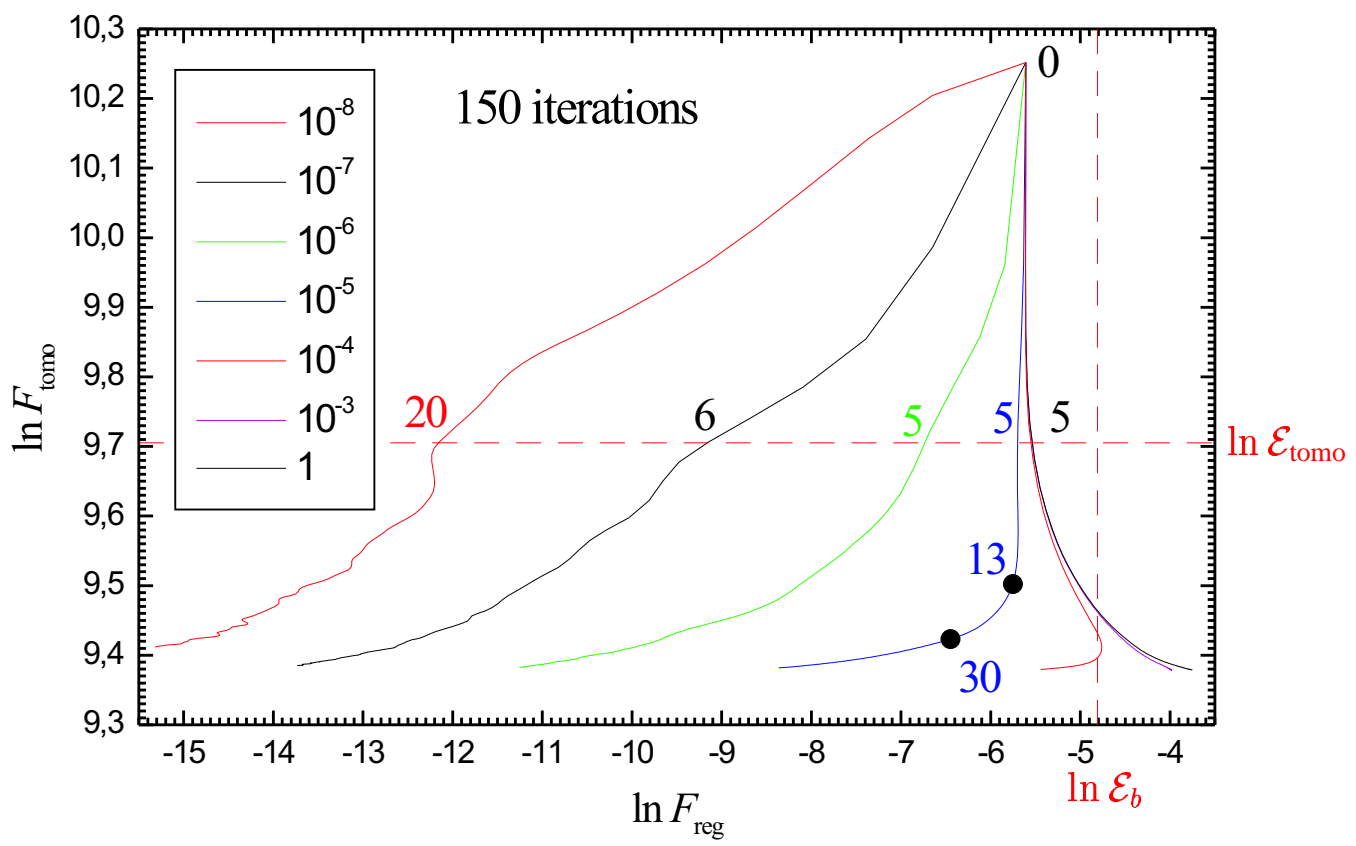

Figure 6.13: The evolution of the logarithm of the data error $F_{\text {tomo }}$ versus the logarithm of the regularization term $F_{\text {reg }}$ during the iterations for different values of $\mu$ and for the reconstruction based on the Hanle-effect data. The Zeeman-effect solution Z1 was used as initial field in the iterations. The colored curves correspond to the values of $\mu$ listed in the insert. The colored numbers near the lines are the number of iterations which were needed to reach the data noise level $\mathcal{E}_{\text {tomo }}$ (red dashed horizontal line). $\mathcal{E}_{b}$ is the boundary noise level in the regularization term. 
6.6.

The Fig. 6.13 shows the behavior of the logarithm of the data error, $F_{\text {tomo }}$, versus the logarithm of the regularization term, $F_{\text {reg }}$, during the iterations for different values of $\mu$. The horizontal dashed line is the data noise level $\mathcal{E}_{\text {tomo }}$ calculated analogously to the case of the Zeeman-effect (equation (6.7)), and $\mathcal{E}_{\mathrm{b}}$ marks the boundary data noise level in the regularization term. In order to obtain a tomography's data error $F_{\text {tomo }} \sim \mathcal{E}_{\text {tomo }}$ and a regularization error $F_{\text {reg }} \sim \mathcal{E}_{\mathrm{b}}$, like for the case of Zeeman-effect data, we had to use $\mu=10^{-5}$ and to stop after 5 iterations (ZH1).

The comparison of this solution with the related reconstruction Z1 based on the Zeemaneffect data (obtained after 47 iterations with $\mu=10^{-3}$ and depicted in the Figs. A.4a,b,c) is presented by the histogram in the Fig. 6.14a, and comparison with the related reconstruction $\mathrm{H} 1$ based on the Hanle-effect data when the potential field approximation was used as initial field (after 7 iterations, figures A.6a,b,c) is presented by the histogram in the Fig. 6.14b. From the plots in the Fig. 6.14 it is difficult to say which of the two Hanle-effect reconstructions is better: the one where Zeeman-effect solution was used as initial condition or the other with potential field approximation as initial field. But, these Hanle solutions represent mainly the main dipole field. For its reconstruction is sufficient to apply the potential field approximation method.

As we are interested in the reconstruction of the non-potential part of the magnetic field, we restrict here our comparison to the perturbed area. To find the iteration number when we have the better reconstruction for the perturbed area, we again like in previous sections calculate the data noise level $\mathcal{E}_{\text {tomo }}^{\prime}$ only for the rays passing through the perturbed region. The data error $F_{\text {tomo }}^{\prime}$ calculated only for these rays reaches the level $\ln \mathcal{E}_{\text {tomo }}^{\prime}$ after $\sim 30$ iterations $(\mathrm{ZH} 3)$. Trying to keep the regularization term error the same like for the case of the Zeeman-effect data (i.e., near $\mathcal{E}_{\mathrm{b}}$ ), we have to stop the minimization procedure after 13-th iteration (ZH2-solution). The cross sections of $\boldsymbol{B}$ for this reconstruction are shown in the Fig. A.9a,b,c.

The histogram of the distribution of the magnitudes of the difference vectors $\left(\boldsymbol{B}_{\text {rec }}-\right.$
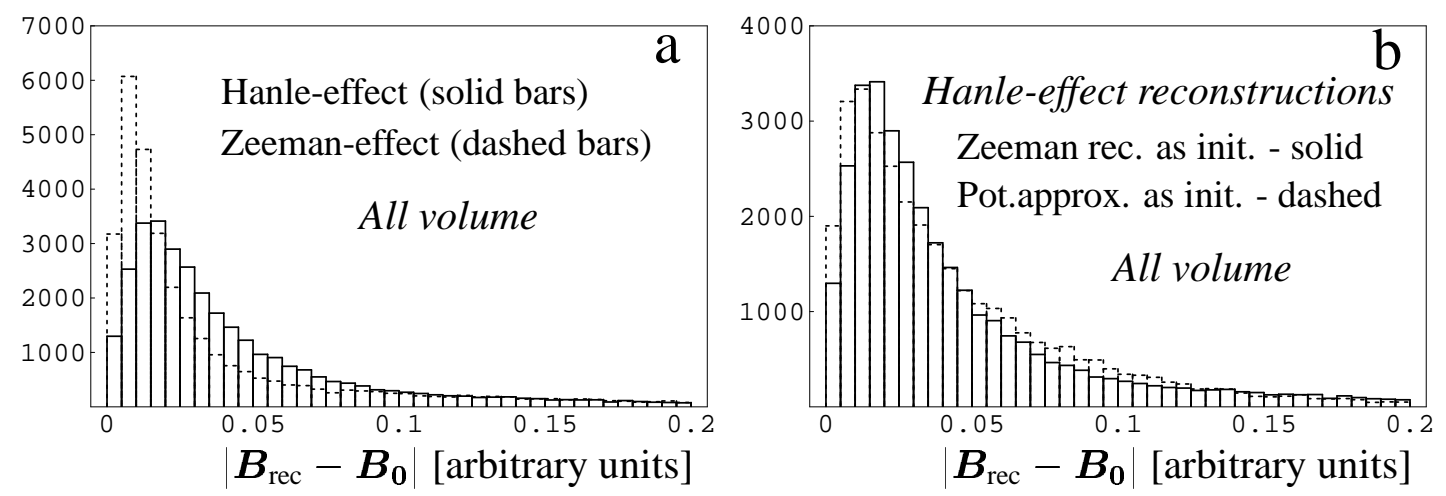

Figure 6.14: The histograms of the distribution of the difference vector magnitudes over the all volume: (a) - for the reconstructions H1 (Hanle-effect data, $\mu=10^{-5}, 5$ iterations, solid bars) and Z1 (Zeeman-effect data, $\mu=10^{-3}, 47$ iterations, dashed bars); (b) - for the reconstructions both based on the Hanle-effect data, but one is ZH1 (solid bars) and other is H1 (dashed bars). For comparison, the minimal value of the magnitude of the model field $\boldsymbol{B}_{\mathbf{0}}$ is $\sim 1$. 

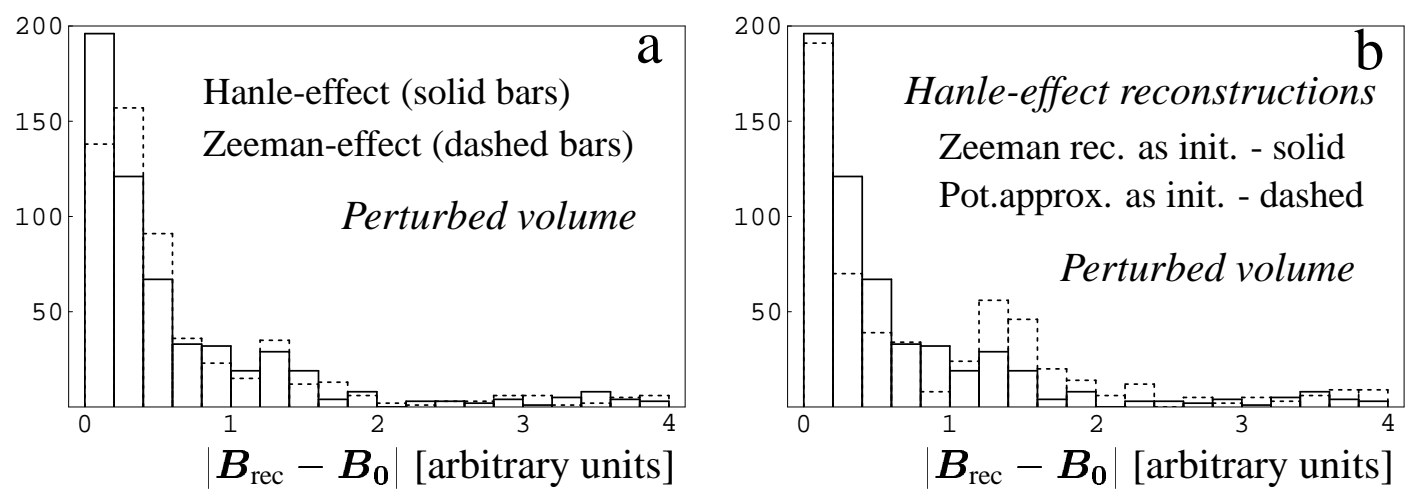

Figure 6.15: The histograms of the distribution of the difference vector magnitudes over the perturbed volume. The solid bars on the both panels represent the Hanle-effect solution $\mathrm{ZH} 2$ for which the $\mathrm{Z} 1$ solution (dashed bars on the panel (a)) was used as initial field. The dashed bars on the panel (b) represent the Hanle-effect solution $\mathrm{H} 2$ whereby the potential field approximation was used as initial condition. For comparison, the average value of the magnitudes of the model field vectors $\boldsymbol{B}_{\mathbf{0}}$ in the perturbed region is $\approx 3.9$.

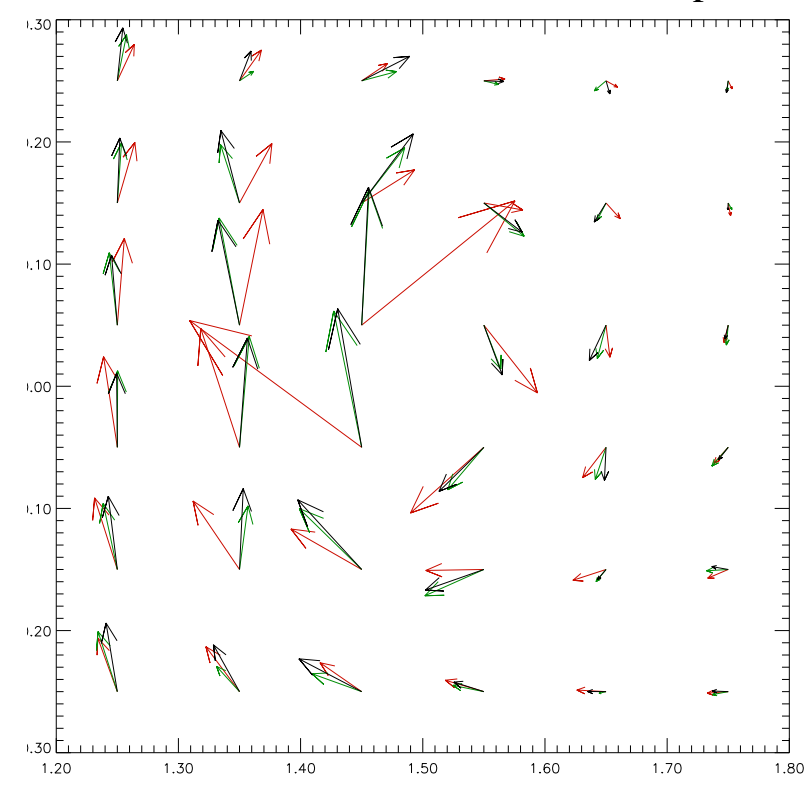

Figure 6.16: The cross sections in the $z=0.15 R_{\odot}$ plane of the model field (red vectors), reconstruction based on the Zeeman-effect (green vectors, Z2-solution), and reconstruction based on the Hanle-effect (black vectors, ZH2-solution).

$\boldsymbol{B}_{\mathbf{0}}$ ) in the perturbed region for this solution is shown in the Fig. 6.15. There are also shown the related histograms for the Zeeman-effect reconstruction Z2 giving the better reconstruction of the perturbed area (with $\mu=10^{-3}$ and stopped after 99 iterations, panel $a$ ) and, in the panel $b$, for the Hanle-effect reconstruction $\mathrm{H} 2$ whereby the potential field approximation was used as initial conditions $\left(\mu=10^{-5}\right.$, after 20 iterations, Sections 6.6 and 6.7). We see that the reconstruction procedure based on the Hanle-effect data and applied after the Zeeman-effect solution gives a better approximation to the model field than when the potential field approximation was used as initial field configuration in the iterations. 


\section{Conclusion}

The goal of the thesis was to investigate the possibility to use the observations of the longitudinal Zeeman effect and/or Hanle effect in coronal emission lines for a reconstruction of the coronal magnetic field. The tomographic inversion problem which we had to solve is considerably more difficult than the traditional scalar tomography inversion problems.

For example, from longitudinal Zeeman-effect data alone, even if we assume the simplifying magnetograph formula to hold, we can only determine the solenoidal part of a vector field $\boldsymbol{V}$, which is a product of the magnetic field with a density and temperature dependent weight function. The knowledge of the sources of $\boldsymbol{V}$, or likewise the fact that $\nabla \cdot \boldsymbol{B}=0$, does not completely cure the problem since a potential field contribution to $\boldsymbol{V}$ still remains undetermined. The incorporation of $\nabla \cdot \boldsymbol{B}=0$ in our inversion procedure as a regularization constraint, however, remedies these two deficits of the Zeeman effect data: $\nabla \cdot \boldsymbol{B}$ is forced to become zero inside our computational domain (at least within the numerical precision of our code) and the calculation of $\nabla \cdot \boldsymbol{B}$ at the domain boundary allows to integrate photospheric magnetic field observations into the inversion in a natural way. Since this photospheric field enforces a boundary condition on our magnetic field solution, the unknown potential field contribution of the solution is uniquely specified.

The theoretical predictions whether an inversion of the Hanle-effect data would be possible are less obvious. The Hanle-effect data of coronal long-lived line transitions (resonance scattering) which we considered in this thesis give no information about the magnitude of the field nor its unique direction. It only yields a weighted line-of-sight integral of the orientation of the magnetic field. Obviously, it is the photospheric magnetic field data included in our inversion code which decides about the magnitude and the sign of the solution magnetic field.

From these considerations it is clear that a data set for the reconstruction of the coronal field from coronal Hanle and longitudinal Zeeman effect data must be supplemented by photospheric normal magnetic field observations to possibly give a unique reconstruction. We note that the reconstruction of the coronal field from the photospheric data alone requires to solve an extrapolation problem, which has its own difficulties and limits as we briefly mentioned in the introduction. Hence a complete data set needed to achieve a reconstruction of the coronal magnetic field with our method comprises:

- Spectropolarimetric Zeeman and/or polarimetric Hanle effect measurements above the Sun's limb for half a solar rotation, i.e., for about 14 days.

- A solar surface magnetogram of the vertical photospheric magnetic field component for the entire surface of the Sun.

While solar surface magnetograms today are standard observational results and are readily 
available, the coronal Hanle effect and especially the coronal Zeeman effect observations are still rare. The experimental effort is considerable and the Zeeman-effect observations have only been successfully performed recently. Ideally, Hanle- and Zeeman-effect observations are made for the same line by a spectropolarimetric measurement of all four Stokes components as in Lin et al.(2004) (see also Fig. 1.1).

The time cadence and the spatial resolution of the observations have great influence on the spatial resolution of the reconstructed magnetic field solution. A precise relationship is difficult to give but could be determined empirically with test calculations similar to those presented here. We also have not addressed the problem of how a non-stationary coronal magnetic field contaminates our solution which is obtained under the strict assumption of stationarity.

In this thesis we demonstrated by means of test calculations that an inversion of the coronal Hanle- and longitudinal Zeeman-effect data in the way proposed above is possible. We have developed a numerical tomography inversion code to perform the test calculations. The code has been written in Fortran and parallelized with OpenMP (shared memory parallelization). The code so far has been used with artificial data only but it is ready for use of real data. Improvements with respect to computation time and memory requirements need to be made for an efficient use. Also the numerical algorithm may be accelerated if a more effective preconditioning scheme is implemented with the conjugate gradient method employed for the minimization procedure. Most of the test calculations presented in this thesis were performed for a corona discretized on a rectangular grid of $40 \times 40 \times 40$ cells, the artificial observations were represented by 36 images from equally spaced viewing angles and with a spatial pixel resolution matched to the resolution of the coronal grid. An inversion of this resolution requires $\sim 260$ Mbytes of storage and a computation time (on a single $1.28 \mathrm{GHz}$ UltraSPARC-IIIi processor) of about $1.5 \mathrm{~s}$ per iteration step if the Zeeman effect data is used. For Hanle-effect data the computation time increases to 6 minutes per iteration, because the inversion problem in this case becomes nonlinear.

Using this inversion code, we have in this thesis only investigated a single model field configuration. This model comprised a background solar dipole field with a north-south orientated coronal current embedded. While the potential field component (essentially the dipole field) can be reproduced from the photospheric surface observations alone by use of Green's theorem, the field perturbations due to the current loop are practically impossible to be reconstructed by extrapolation methods. The main test of our calculations therefore was to find out the quality with which this perturbation field was reproduced. As it was emphasized in the introduction, this non-potential part of the coronal field is decisive for a quantitative understanding of many coronal processes which are often driven by a loss of stability of the coronal magnetic field configuration.

Good agreement between the model field and our field reconstruction were obtained when we used artificial Zeeman effect data as input. Hanle effect data gave less satisfactory results. We presume that the reason is the north-south geometry of the current loop. The perturbation magnetic field of this current produces a much stronger response in the longitudinal Zeeman than in the Hanle effect observations. This should be different for current loops with east-west orientation which should better be visible in the Hanle-effect data. These latter loops should occur more often at lower height $\left(\lesssim 0.2 R_{\odot}\right)$ as active bipolar regions are mostly orientated in east-west direction and the dominant 
current should flow along the magnetic field lines. Older bipolar regions however are tilted into the meridional direction by the differential rotation of the Sun's surface. As an example of a near-meridional loop, Fig. 7.1(a) shows an EIT image of a magnetic flux closure across the equator between two active regions. The field which was used as a test field (Fig. 7.1b,c) shows very similar deformations near the current loop. Note, however, that our test field model is not force free. Also, at higher latitudes and higher altitudes above the surface, coronograph observations suggest that streamer-type loop systems are common which are also close to the test field model. The test simulations have shown that:

- The tomographic inversion based on the data for the Hanle effect and(or) longitudinal Zeeman effect, and on the constraint $\nabla \cdot \boldsymbol{B}=0$, allows one to reconstruct the non-potential component of the magnetic field, while a traditional potential field approximation reconstructs only the potential field component.

- The tomographic inversions based on the Hanle effect and longitudinal Zeeman effect, have different precision for the different vector components of the field, depending on the configuration of the reconstructing field. Particularly, for the case of observation of a vortex-like field situated in the plane perpendicular to the rotation axis, the vortex is hardly seen in the reconstruction based on the Hanle effect, while the reconstruction based on the Zeeman effect gives satisfactory result for this field. The inversion based on the Hanle effect gives more precise result for the meridional component of the magnetic field than an inversion based on the Zeeman effect. 

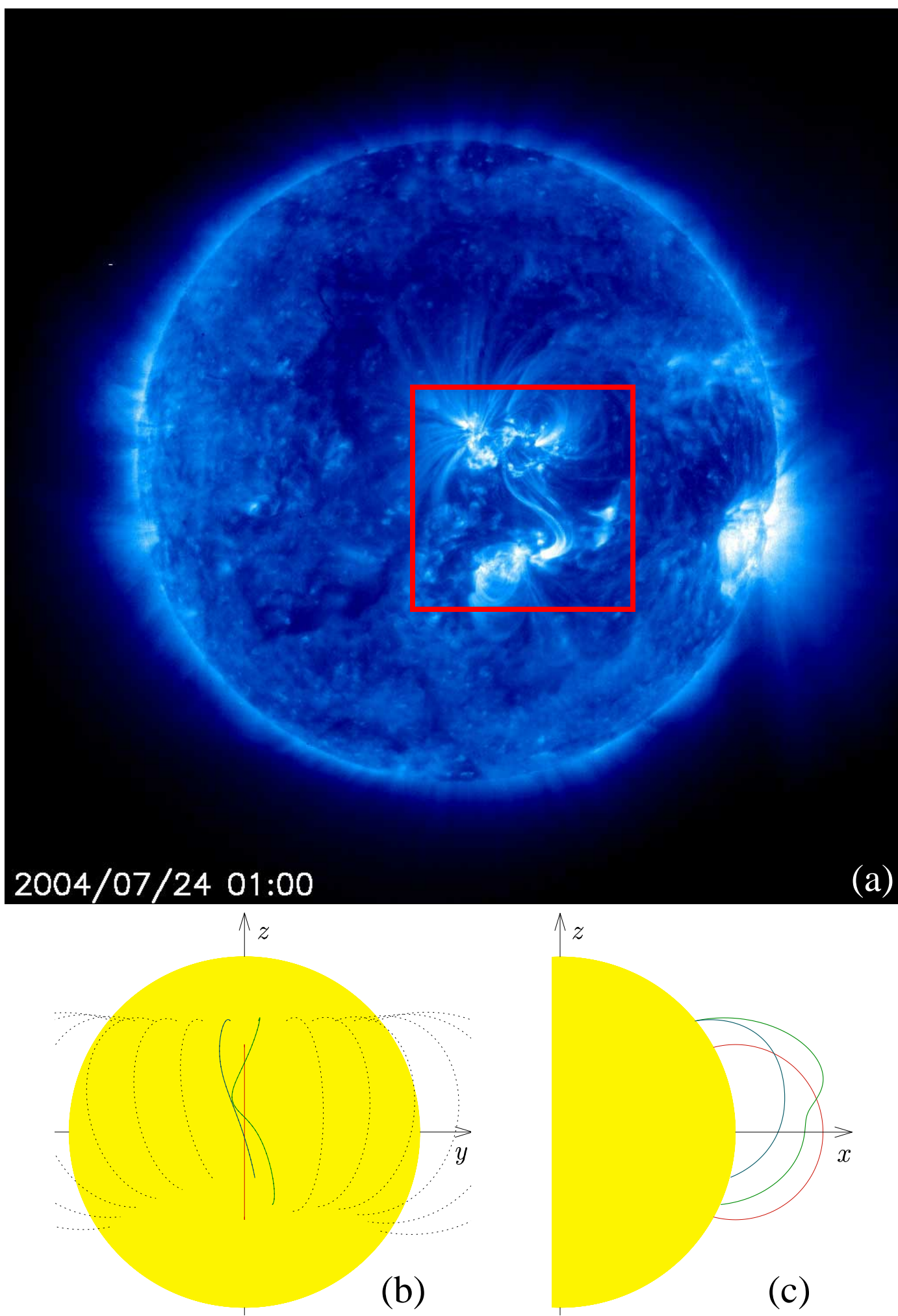

Figure 7.1: An EUV image made by the EIT instrument on board the SOHO spacecraft (a). The image was taken at the emission line of Fe XII at $\lambda=171 \AA$. It shows a magnetic flux closure across the equator between two active regions (inside red box), and field lines of the test model (lover pictures). Panel (b) shows the view from the $x=+\infty$ direction, and (c) shows the view from the $y=-\infty$ direction. The most perturbed field lines are marked by blue and green colors. The perturbing current loop is shown by the red curve. 


\section{Outlook}

The results produced by the code developed in this thesis demonstrated that it is in principle possible to reconstruct the coronal magnetic field from coronal Hanle-effect and/or Zeeman-effect data. The present study is to our knowledge the first investigation which uses tomographic inversion techniques for this task. However, this thesis can only be a first step and several questions remain to be answered and further improvements should be made:

- As mentioned above, different and more realistic coronal magnetic field configurations, e.g., the field above active regions or more realistic streamer-type field structures should be studied to find out which details of the field can or cannot be reconstructed and how much the Hanle effect or longitudinal Zeeman effect observations contribute to the respective solutions. It would also be interesting to find out how much is gained if both observations were available.

- A common problem when inversion codes are applied to real data is the influence of the instrument noise on the solution. With the code we have developed, we can study with test calculations systematically how much noise is tolerable to achieve a certain precision of the solution. The signal-to-noise ratio that we obtain by these means can then be used to determine the integration time for the coronal Hanle and Zeeman effect observations. Since the integration time is about an hour for typical observations of this kind, this information could be highly relevant.

- Another topic that should be investigated is the influence of data gaps on the inversion result. Since the Hanle and Zeeman effect observations are made from the ground, missing viewing directions may well occur during the 14-day observing sequence. Gaps in the data obviously reduce the quality of the inversion result but is not obvious how sensitively the result is affected by them.

- Observations of the Faraday rotation of the linearly polarized radio signals traveling through the corona give information very similar to the longitudinal Zeeman effect. However, the data is obtained at a given time only along a single line of sight rather than on a whole set of lines of sight corresponding to the pixels of an image. It would be interesting to study how useful these sparse measurements are for the reconstruction of the coronal field. A one of the examples for coronal Faradayrotation observations are the measurements of Jensen et al. (2005), who used the radio signal of the Cassini spacecraft during its passage behind the Sun as the radio source. 
- In the code used in this thesis we neglected the alignment factor (see eq. (5.56)). As discussed in Section 5.12, a finite alignment factor will modify the numerical expressions for the inversion of the longitudinal Zeeman-effect data by about $30 \%$ or less. For a quantitative application of our code to real data, a calculation of the alignment factor should be included.

- The inversion procedure presented here could be looked at as a first step towards a systematic line-of-sight inversion of all four Stokes components which would then yield not only the magnetic field but also the coronal density (mainly from the Stokes- $I$ component)

For many issues on this list, the inversion code developed here can be used to find an empirical answer. 


\section{A Appendix}

\section{A.1 Potential field approximation}

The potential field approximation is often used to reconstruct the magnetic field in the solar corona on the basis of measurements of the magnetic field at the photospheric level. The assumption for this model is that there are no electric currents above the photosphere, i.e.,

$$
\nabla \times \boldsymbol{B}=0 .
$$

In this case the magnetic field can be written as the gradient of a scalar potential $\Phi$,

$$
\boldsymbol{B}=-\nabla \cdot \Phi \text {. }
$$

Since $\nabla \cdot \boldsymbol{B}=0$, we need to solve Laplace equation

$$
\nabla^{2} \Phi=0
$$

Gauss (1839) showed that having the radial component of the magnetic field, $B_{r}$, on the photosphere is a sufficient boundary condition, and the expansion of the potential $\Phi$ in spherical harmonics, $Y_{l m}$, gives a complete solution:

$$
\Phi(r, \theta, \phi)=\sum_{l=0}^{\infty} \sum_{m=-l}^{l}\left(A_{l m} r^{l}+B_{l m} r^{-(l+1)}\right) \cdot Y_{l m}(\theta, \phi)
$$

with the spherical harmonics expressed through the associated Legendre polynomials, $P_{l}^{m}(\cos \theta)$, by the equation

$$
Y_{l m}(\theta, \phi)=\sqrt{\frac{2 l+1}{4 \pi} \frac{(l-m) !}{(l+m) !}} P_{l}^{m}(\cos \theta) \mathrm{e}^{\mathrm{i} m \phi}
$$

The coefficients $A_{l m}$ and $B_{l m}$ must be calculated from the boundary conditions. If we suppose $|\boldsymbol{B}|(r \rightarrow \infty)=0$ (no magnetic field sources above the photosphere), then

$$
\begin{array}{r}
A_{l m}=0, \\
B_{l m}=-\frac{C_{l m} r_{0}^{l+2}}{l+1},
\end{array}
$$

where

$$
C_{l m}=\int_{0}^{2 \pi} \int_{0}^{\pi}(-1)^{m} Y_{l,-m}(\theta, \phi) B_{r}\left(r_{0}, \theta, \phi\right) \sin (\theta) d \theta d \phi
$$


This model does not represent a real magnetic field configuration since observations show that the coronal magnetic field is dominantly radial above approximately two solar radii. To improve the situation, Schatten, Wilcox and Ness (1969) introduced a source sphere at distance $r_{1}$ from the solar surface where the magnetic field becomes radial. In this case the coefficients $A_{l m}$ and $B_{l m}$ for $l \geq 1$ are:

$$
\begin{gathered}
A_{l m}=\frac{C_{l m} r_{0}^{2+l}}{r_{1}^{1+2 l}+l\left(r_{0}^{1+2 l}+r_{1}^{1+2 l}\right)}, \\
B_{l m}=-\frac{C_{l m} r_{0}^{2+l} r_{1}^{1+2 l}}{r_{1}^{1+2 l}+l\left(r_{0}^{1+2 l}+r_{1}^{1+2 l}\right)} .
\end{gathered}
$$

For $l=0$ the coefficient $A_{00}$ can be chosen arbitrary and

$$
B_{00}=-\frac{C_{00} r_{0}^{2}}{\sqrt{4 \pi}}=0 \quad \text { (there is no magnetic monopole allowed). }
$$

There are several ways to find the radial component of the magnetic field vector at the solar surface from magnetogram measurements (MDI). The magnetogram gives the LOS component of the magnetic field at the photosphere. Suppose the magnetic field is constant during solar rotation and on average radial at the photosphere. Then, by measuring the LOS component of the field, $B^{\mathrm{LOS}}$, during the central meridian passage, one finds that

$$
B_{r}=\frac{B^{\mathrm{LOS}}}{\sin \theta}
$$

where $\theta$ is the latitude $(0<\theta<\pi)$.

In the second way, it is also assumed that the magnetic field is constant during a solar rotation, but it has no radial structure. Let us consider a region at longitude $\phi$ with respect to the central meridian, and latitude $\theta$ (Fig. A.1). It is possible to measure $B_{i}^{\mathrm{LOS}}$ for this particular region for at several positions $\phi_{i}$. So, we have a system of linear equations for a single region:

$$
B_{r} \sin \theta \cos \phi_{i}+B_{\theta} \cos \theta \cos \phi_{i}-B_{\phi} \sin \phi_{i}=B_{i}^{\mathrm{LOS}},
$$

where $B_{\theta}$ and $B_{\phi}$ are the latitudinal and longitudinal components of the field vector $\boldsymbol{B}$, respectively. Then, if we suppose that the magnetic field is axisymmetric $\left(B_{\phi}=0\right)$, and measuring LOS component, $B^{\mathrm{LOS}}$, during a central meridian passage $\left(\phi_{i}=0\right)$, the values of the $B_{r}$ and $B_{\theta}$ can be found from (A.13) by least-square fitting (for example, by SVD-method). Here the inclination of the Sun's pole was not taken into account.

\section{A.2 Force-free field reconstruction}

The force-free magnetic field approximation is valid when the electrical currents are parallel to the magnetic field. This occurs in the low corona where the plasma $\beta$ is small. A force-free magnetic field must satisfy the equations:

$$
\begin{array}{r}
\nabla \cdot \boldsymbol{B}=0, \\
4 \pi \boldsymbol{J}=\nabla \times \boldsymbol{B}=\alpha \boldsymbol{B},
\end{array}
$$



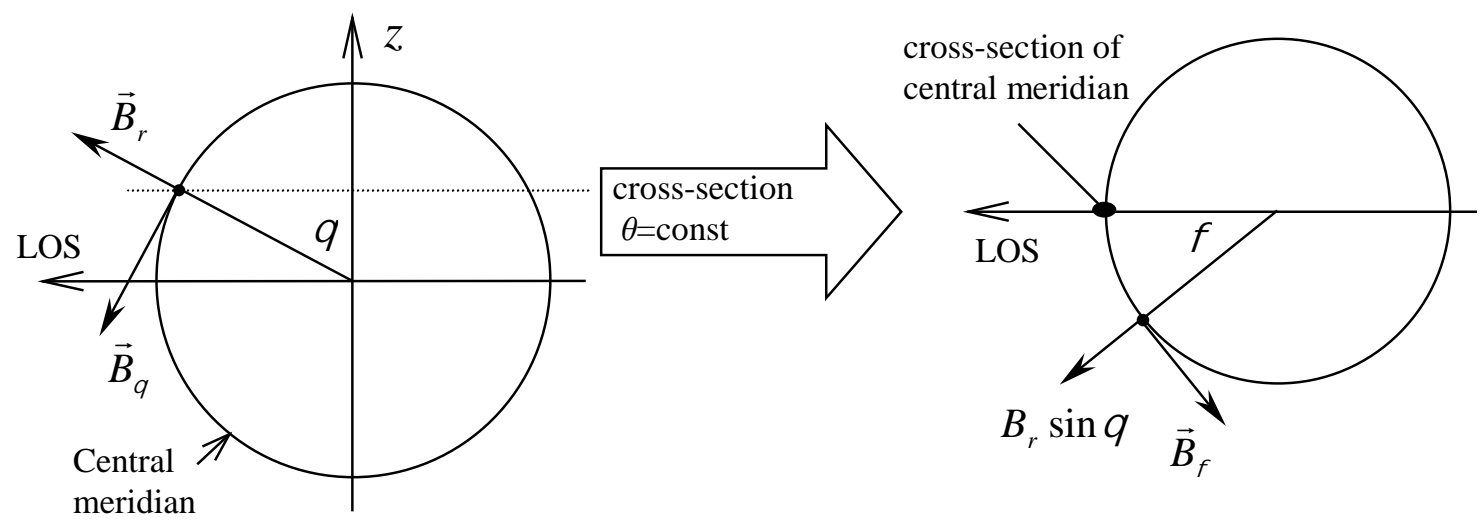

Figure A.1: Definition of field components at the photospheric level.

where $\boldsymbol{J}$ is current density, and $\alpha$ is function of the spatial coordinates. Taking the divergence of (A.15) and using (A.14) one obtains

$$
\boldsymbol{B} \cdot \nabla \alpha=0,
$$

which shows that $\alpha$ is constant along individual field lines.

Solution (A.14) and (A.15) require a magnetic field at the photospheric level as boundary condition. The case of constant $\alpha$, the linear force-free model, has been widely investigated by plenty of authors (Nakagawa et al. 1971; Nakagawa \& Raadu 1972; Seehafer 1978; Alissandrakis 1981; Chiu \& Hilton 1977; Semel 1988).

However, the linear force-free model is still far from representing a real magnetic field configuration. Observations show that $\alpha$ is not constant even within a single active region (Levine 1976; Krall et al. 1982; Schmahl et al. 1982; Gary 1987). Therefore the nonlinear force-free model with non-constant $\alpha$ is more realistic (Sakurai 1981; Cuperman et al. 1990; Wiegelmann 2004; Weatland 2004).

\section{A.3 Michelson Doppler Imager (MDI)}

The Michelson Doppler Imager (MDI) on SOHO makes images of the Sun on a CCD camera with resolution of $1024 \times 1024$ pixels. The filter system of the instrument (front window, the blocker, the Lyot and two wide-field tunable Michelson interferometers) allows to make narrow-band (94 mA) image near the Ni I $6768 \AA$ photospheric absorption line. Images are obtained at five fixed wavelength equally separated by $75 \mathrm{~mA}$ with intensities $I_{0}, I_{1}, I_{2}, I_{3}$ and $I_{4} . I_{0}$ is near continuum, $I_{1}$ and $I_{4}$ is centered on the wings, and $I_{2}$ and $I_{3}$ is centered near the core of the $\mathrm{Ni}$ I line at the center of solar disk. The depth of the $\mathrm{Ni}$ I line is the continuum intensity minus the line-center one and compute from the four images:

$$
I_{\text {depth }}=\sqrt{2\left(\left(I_{1}-I_{3}\right)^{2}+\left(I_{2}-I_{4}\right)^{2}\right)} .
$$

The continuum intensity near the $\mathrm{Ni}$ I line is

$$
I_{\mathrm{c}}=2 I_{0}+I_{\mathrm{depth}} / 2+\left(I_{1}+I_{2}+I_{3}+I_{4}\right) / 4 \text {. }
$$


The Doppler shift (velocity) is computed from a ratio

$$
\begin{aligned}
& \left(I_{1}+I_{2}-I_{3}-I_{4}\right) /\left(I_{1}-I_{3}\right), \text { if numerator }>0, \\
& \left(I_{1}+I_{2}-I_{3}-I_{4}\right) /\left(I_{4}-I_{2}\right), \text { if numerator } \leq 0,
\end{aligned}
$$

using results obtained from parameterized solar line profiles and the measured filter transmission profiles (Scherrer et al. 1995).

The difference between the Doppler shifts taken separately in right and left circularly polarized light is roughly proportional to the LOS-component of the magnetic field averaged over the resolution element. This operation is made by inserting the polarizers.

A full-disk image has a plate scale of $2^{\prime \prime}$ per pixel and a resolution of $4^{\prime \prime}$. Also, MDI produces a focused image of an $11^{\prime}$ square field with higher resolution $\left(1.25^{\prime \prime}\right)$ and a plate scale of $0.625^{\prime \prime}$ per pixel. 


\section{A.4 Spherical tensor $\mathcal{T}$ for polarimetry of magnetic dipole transitions in reducible representation}

\begin{tabular}{|c|c|c|}
\hline $\mathcal{T}_{-1,-1}(0, \Omega)$ & $=$ & $\frac{1}{4}\left(1+\cos ^{2} \theta\right)$ \\
\hline $\mathcal{T}_{0,0}(0, \Omega)$ & $=$ & $\frac{1}{2} \sin ^{2} \theta$ \\
\hline $\mathcal{T}_{1,1}(0, \Omega)$ & $=$ & $\frac{1}{4}\left(1+\cos ^{2} \theta\right)$ \\
\hline $\mathcal{T}_{-1,0}(0, \Omega)$ & $=$ & $-\frac{1}{2 \sqrt{2}} \sin \theta \cos \theta \mathrm{e}^{-\mathrm{i} \chi}$ \\
\hline $\mathcal{T}_{-1,1}(0, \boldsymbol{\Omega})$ & $=$ & $\frac{1}{4} \sin ^{2} \theta \mathrm{e}^{-2 \mathrm{i} \chi}$ \\
\hline $\mathcal{T}_{0,1}(0, \Omega)$ & $=$ & $\frac{1}{2 \sqrt{2}} \sin \theta \cos \theta \mathrm{e}^{-\mathrm{i} \chi}$ \\
\hline $\mathcal{T}_{-1,-1}(1, \Omega)$ & $=$ & $\frac{1}{4} \cos 2 \gamma \sin ^{2} \theta$ \\
\hline $\mathcal{T}_{0,0}(1, \Omega)$ & $=$ & $-\frac{1}{2} \cos 2 \gamma \sin ^{2} \theta$ \\
\hline $\mathcal{T}_{1,1}(1, \Omega)$ & $=$ & $\frac{1}{4} \cos 2 \gamma \sin ^{2} \theta$ \\
\hline $\mathcal{T}_{-1,0}(1, \Omega)$ & $=$ & $\frac{1}{2 \sqrt{2}}(\cos 2 \gamma \cos \theta-\mathrm{i} \sin 2 \gamma) \sin \theta \mathrm{e}^{-\mathrm{i} \chi}$ \\
\hline $\mathcal{T}_{-1,1}(1, \boldsymbol{\Omega})$ & $=$ & $\frac{1}{4}\left(\cos 2 \gamma\left(1+\cos ^{2} \theta\right)-2 \mathrm{i} \sin 2 \gamma \cos \theta\right) \mathrm{e}^{-2 \mathrm{i} \chi}$ \\
\hline $\mathcal{T}_{0,1}(1, \Omega)$ & $=$ & $-\frac{1}{2 \sqrt{2}}(\cos 2 \gamma \cos \theta-\mathrm{i} \sin 2 \gamma) \sin \theta \mathrm{e}^{-\mathrm{i} \chi}$ \\
\hline $\mathcal{T}_{-1,-1}(2, \Omega)$ & $=$ & $-\frac{1}{4} \sin 2 \gamma \sin ^{2} \theta$ \\
\hline $\mathcal{T}_{0,0}(2, \boldsymbol{\Omega})$ & $=$ & $\frac{1}{2} \sin 2 \gamma \sin ^{2} \theta$ \\
\hline $\mathcal{T}_{1,1}(2, \Omega)$ & $=$ & $-\frac{1}{4} \sin 2 \gamma \sin ^{2} \theta$ \\
\hline $\mathcal{T}_{-1,0}(2, \Omega)$ & $=$ & $-\frac{1}{2 \sqrt{2}}(\sin 2 \gamma \cos \theta+\mathrm{i} \cos 2 \gamma) \sin \theta \mathrm{e}^{-\mathrm{i} \chi}$ \\
\hline $\mathcal{T}_{-1,1}(2, \Omega)$ & $=$ & $-\frac{1}{4}\left(\sin 2 \gamma\left(1+\cos ^{2} \theta\right)+2 \mathrm{i} \cos 2 \gamma \cos \theta\right) \mathrm{e}^{-2 \mathrm{i} \chi}$ \\
\hline $\mathcal{T}_{0,1}(2, \Omega)$ & $=$ & $\frac{1}{2 \sqrt{2}}(\sin 2 \gamma \cos \theta+\mathrm{i} \cos 2 \gamma) \sin \theta \mathrm{e}^{-\mathrm{i} \chi}$ \\
\hline $\mathcal{T}_{-1,-1}(3, \boldsymbol{\Omega})$ & $=$ & $-\frac{1}{2} \cos \theta$ \\
\hline $\mathcal{T}_{0,0}(3, \Omega)$ & $=$ & 0 \\
\hline $\mathcal{T}_{1,1}(3, \Omega)$ & $=$ & $\frac{1}{2} \cos \theta$ \\
\hline $\mathcal{T}_{-1,0}(3, \boldsymbol{\Omega})$ & $=$ & $\frac{1}{2 \sqrt{2}} \sin \theta \mathrm{e}^{-\mathrm{i} \chi}$ \\
\hline $\mathcal{T}_{-1,1}(3, \boldsymbol{\Omega})$ & $=$ & 0 \\
\hline $\mathcal{T}_{0,1}(3, \boldsymbol{\Omega})$ & $=$ & $\frac{1}{2 \sqrt{2}} \sin \theta \mathrm{e}^{-\mathrm{i} \chi}$ \\
\hline
\end{tabular}

Table A.1: Expressions for the spherical tensor $\mathcal{T}_{q q^{\prime}}(i, \Omega)$ for lines corresponding to magnetic dipole transitions (Landi \& Landolfi 2004). It should be noted that $\mathcal{T}_{q^{\prime} q}(i, \Omega)=$ $\mathcal{T}_{q q^{\prime}}(i, \boldsymbol{\Omega})^{*}$. 


\section{A.5 Wigner symbols}

In this section, we give brief introduction into the Wigner 3- $j, 6-j$, and 9- $j$ symbols and describe some basic properties of them. More detailed describtion of the symbols and theory connected with them can be found in Zare (1988), Varshalovich et al. (1989).

\section{A.5.1 Wigner 3- $j$ symbol}

The Wigner 3- $j$ symbol,

$$
\left(\begin{array}{ccc}
j_{1} & j_{2} & j_{3} \\
m_{1} & m_{2} & m_{3}
\end{array}\right)
$$

describes the coupling of two angular momenta in quantum mechanics. Here, $j_{1}, j_{2}$, and $j_{3}$ are the eigenvalues of the angular momentum operator, and $m_{1}, m_{2}$, and $m_{3}$ are the eigenvalues of their projections onto the quantization axis $z$, respectively. The $3-j$ symbol is related to the Clebsch-Gordan coefficients by

$$
\left(\begin{array}{ccc}
j_{1} & j_{2} & j_{3} \\
m_{1} & m_{2} & m_{3}
\end{array}\right)=(-1)^{j_{3}+m_{3}+2 j_{1}} \frac{1}{\sqrt{2 j_{3}+1}} C_{j_{1},-m_{1}, j_{2},-m_{2}}^{j_{3}, m_{3}}
$$

and inverse relation

$$
C_{j_{1}, m_{1}, j_{2}, m_{2}}^{j_{3}, m_{3}}=(-1)^{j_{1}-j_{2}+m_{3}} \sqrt{2 j_{3}+1}\left(\begin{array}{ccc}
j_{1} & j_{2} & j_{3} \\
m_{1} & m_{2} & -m_{3}
\end{array}\right) .
$$

A Clebsch-Gordan coefficient, $C_{j_{1}, m_{1}, j_{2}, m_{2}}^{j_{3}, m_{3}}$, represents the probability amplitude that $j_{1}$ and $j_{2}$ with their projections $m_{1}$ and $m_{2}$ are coupled into the resultant angular momentum $j_{3}$ with the projection $m_{3}$.

The coefficients in the $3-j$ symbol satisfy the follow selection rules:

- $m_{1}=-\left|j_{1}\right|, \ldots,\left|j_{1}\right|, m_{2}=-\left|j_{2}\right|, \ldots,\left|j_{2}\right|$, and $m_{3}=-\left|j_{3}\right|, \ldots,\left|j_{3}\right|$.

- $m_{1}+m_{2}+m_{3}=0$.

- $\left|j_{1}-j_{2}\right| \leq j_{3} \leq\left(j_{1}+j_{2}\right)$ (triangular inequality).

- $j_{1}+j_{2}+j_{3}$ is an integer.

If these conditions are not satisfied, then the $3-j$ symbol (A.20) is zero.

The $3-j$ symbols satisfy the orthogonality relations:

$$
\begin{gathered}
\sum_{m_{1}, m_{2}}\left(\begin{array}{ccc}
j_{1} & j_{2} & j_{3} \\
m_{1} & m_{2} & m_{3}
\end{array}\right)\left(\begin{array}{ccc}
j_{1} & j_{2} & j_{3}^{\prime} \\
m_{1} & m_{2} & m_{3}^{\prime}
\end{array}\right)=\left(2 j_{3}+1\right)^{-1} \delta_{j_{3} j_{3}^{\prime}} \delta_{m_{3} m_{3}^{\prime}} \\
\sum_{j_{3}, m_{3}}\left(2 j_{3}+1\right)\left(\begin{array}{ccc}
j_{1} & j_{2} & j_{3} \\
m_{1} & m_{2} & m_{3}
\end{array}\right)\left(\begin{array}{ccc}
j_{1} & j_{2} & j_{3} \\
m_{1}^{\prime} & m_{2}^{\prime} & m_{3}
\end{array}\right)=\delta_{m_{1} m_{1}^{\prime}} \delta_{m_{2} m_{2}^{\prime}}
\end{gathered}
$$

An even permutation of the columns leaves the numerical value of the $3-j$ symbol unchanged,

$$
\left(\begin{array}{ccc}
j_{1} & j_{2} & j_{3} \\
m_{1} & m_{2} & m_{3}
\end{array}\right)=\left(\begin{array}{ccc}
j_{2} & j_{3} & j_{1} \\
m_{2} & m_{3} & m_{1}
\end{array}\right)=\left(\begin{array}{ccc}
j_{3} & j_{1} & j_{2} \\
m_{3} & m_{1} & m_{2}
\end{array}\right)
$$


while an odd permutation is equivalent to multiplication by $(-1)^{j_{1}+j_{2}+j_{3}}$. The replacement of the bottom row by the negative of all its arguments is also equivalent to multiplication by $(-1)^{j_{1}+j_{2}+j_{3}}$.

The $3-j$ symbol can be calculated by

$$
\begin{array}{r}
\left(\begin{array}{ccc}
j_{1} & j_{2} & j_{3} \\
m_{1} & m_{2} & m_{3}
\end{array}\right)=(-1)^{j_{1}-j_{2}-m_{3}} \Delta\left(j_{1} j_{2} j_{3}\right) \times \\
\times \sqrt{\left(j_{1}+m_{1}\right) !\left(j_{1}-m_{1}\right) !\left(j_{2}+m_{2}\right) !\left(j_{2}-m_{2}\right) !\left(j_{3}+m_{3}\right) !\left(j_{3}-m_{3}\right) !} \times \\
\times \sum_{t} \frac{(-1)^{t}}{f(t)}
\end{array}
$$

where the triangular coefficient, $\Delta$, is

$$
\Delta(a b c)=\sqrt{\frac{(a+b-c) !(a-b+c) !(-a+b+c) !}{(a+b+c+1) !}},
$$

and

$$
\begin{array}{r}
f(t)=t !\left(j_{1}+j_{2}-j_{3}-t\right) !\left(j_{1}-m_{1}-t\right) !\left(j_{2}+m_{2}-t\right) ! \times \\
\times\left(j_{3}-j_{2}+m_{1}+t\right) !\left(j_{3}-j_{1}-m_{2}+t\right) !
\end{array}
$$

The sum in (A.26) is over all integers $t$ for which the factorials in $f(t)$ all have nonnegative arguments.

\section{A.5.2 Wigner 6- $j$ symbol}

Three angular momenta $\boldsymbol{j}_{1}, \boldsymbol{j}_{2}$, and $\boldsymbol{j}_{3}$ may be coupled to a resultant angular momentum $j$ in three ways:

1. $\boldsymbol{j}_{1}+\boldsymbol{j}_{2}=\boldsymbol{j}_{12}, \boldsymbol{j}_{12}+\boldsymbol{j}_{3}=\boldsymbol{j}$

2. $\boldsymbol{j}_{2}+\boldsymbol{j}_{3}=\boldsymbol{j}_{23}, \boldsymbol{j}_{1}+\boldsymbol{j}_{23}=\boldsymbol{j}$;

3. $\boldsymbol{j}_{1}+\boldsymbol{j}_{3}=\boldsymbol{j}_{13}, \boldsymbol{j}_{13}+\boldsymbol{j}_{2}=\boldsymbol{j}$.

The Wigner 6- $j$ symbol,

$$
\left\{\begin{array}{ccc}
j_{1} & j_{2} & j_{12} \\
j_{3} & j & j_{23}
\end{array}\right\}
$$

describes the coupling of three angular momenta and can be given through the ClebschGordan coefficients:

$$
\begin{array}{r}
\sum C_{j_{12}, m_{12}, j_{3}, m_{3}}^{j, m} C_{j_{1}, m_{1}, j_{2}, m_{2}}^{j_{12}, m_{12}} C_{j_{1}, m_{1}, j_{23}, m_{23}}^{j^{\prime}, m^{\prime}} C_{j_{2}, m_{2}, j_{3}, m_{3}}^{j_{23}, m_{23}}= \\
=\delta_{j j^{\prime}} \delta_{m m^{\prime}}(-1)^{j_{1}+j_{2}+j_{3}+j} \sqrt{\left(2 j_{12}+1\right)\left(2 j_{23}+1\right)}\left\{\begin{array}{ccc}
j_{1} & j_{2} & j_{12} \\
j_{3} & j & j_{23}
\end{array}\right\} .
\end{array}
$$

Here the sum is over $m_{1}, m_{2}, m_{3}, m_{12}$, and $m_{23}$, while $m$ and $m^{\prime}$ are fixed. The symbol $\delta$ is a Kronecker delta-symbol.

The coefficients in the 6- $j$ symbol (A.29) are either integers or half-integers. Their triads, $\left(j_{1}, j_{2}, j_{12}\right),\left(j_{23}, j_{1}, j\right),\left(j_{2}, j_{3}, j_{23}\right)$, and $\left(j_{12}, j_{3}, j\right)$, must satisfy the following conditions: 
- Each triad satisfies the triangular inequality.

- The sum of the elements of each triad is an integer.

If these conditions are not satisfied, then the 6- $j$ symbol (A.29) is zero.

The 6- $j$ symbol is invariant under permutation of their columns and under interchange of the upper and lower elements in any two columns.

The 6- $j$ symbol can be calculated using the Racah formula:

$$
\begin{array}{r}
\left\{\begin{array}{lll}
a & b & c \\
d & e & f
\end{array}\right\}=\Delta(a b c) \Delta(\text { aef }) \Delta(d b f) \Delta(d e c) \times \\
\times \sum_{t} \frac{(-1)^{t}(t+1) !}{f(t)},
\end{array}
$$

where $\Delta$ is a triangle coefficient (A.27), and

$$
\begin{aligned}
& f(t)=(t-a-b-c) !(t-a-e-f) !(t-d-b-f) !(t-d-e-c) ! \times \\
& \times(a+b+d+e-t) !(b+c+e+f-t) !(a+c+d+f-t) !
\end{aligned}
$$

The sum in (A.31) is over all integers $t$ for which the factorials in $f(t)$ all have nonnegative arguments.

\section{A.5.3 Wigner 9- $j$ symbol}

Four angular momenta, $\boldsymbol{j}_{1}, \boldsymbol{j}_{2}, \boldsymbol{j}_{3}, \boldsymbol{j}_{4}$, can be coupled to give a resultant angular momentum $j$ in the ways:

1. $\boldsymbol{j}_{1}+\boldsymbol{j}_{2}=\boldsymbol{j}_{12}, \quad \boldsymbol{j}_{3}+\boldsymbol{j}_{4}=\boldsymbol{j}_{34}, \quad \boldsymbol{j}_{12}+\boldsymbol{j}_{34}=\boldsymbol{j}$;

2. $\boldsymbol{j}_{1}+\boldsymbol{j}_{3}=\boldsymbol{j}_{13}, \quad \boldsymbol{j}_{2}+\boldsymbol{j}_{4}=\boldsymbol{j}_{24}, \quad \boldsymbol{j}_{13}+\boldsymbol{j}_{24}=\boldsymbol{j}$;

3. $\boldsymbol{j}_{1}+\boldsymbol{j}_{4}=\boldsymbol{j}_{14}, \quad \boldsymbol{j}_{2}+\boldsymbol{j}_{3}=\boldsymbol{j}_{23}, \quad \boldsymbol{j}_{14}+\boldsymbol{j}_{23}=\boldsymbol{j}$.

The Wigner 9- $j$ symbol,

$$
\left\{\begin{array}{ccc}
j_{1} & j_{3} & j_{13} \\
j_{2} & j_{4} & j_{24} \\
j_{12} & j_{34} & j
\end{array}\right\},
$$

is associated with the coefficients of unitary transformations which connect state vectors corresponding to different coupling schemes of four angular momenta, and can be defined through the 3-j symbols:

$$
\begin{aligned}
&\left\{\begin{array}{ccc}
j_{1} & j_{3} & j_{13} \\
j_{2} & j_{4} & j_{24} \\
j_{12} & j_{34} & j
\end{array}\right\}= \\
&=\sum_{\text {All } m}\left(\begin{array}{ccc}
j_{1} & j_{3} & j_{13} \\
m_{1} & m_{3} & m_{13}
\end{array}\right)\left(\begin{array}{ccc}
j_{2} & j_{4} & j_{24} \\
m_{2} & m_{4} & m_{24}
\end{array}\right)\left(\begin{array}{ccc}
j_{12} & j_{34} & j \\
m_{12} & m_{34} & m
\end{array}\right) \times \\
& \times\left(\begin{array}{cccc}
j_{1} & j_{2} & j_{12} \\
m_{1} & m_{2} & m_{12}
\end{array}\right)\left(\begin{array}{cccc}
j_{3} & j_{4} & j_{34} \\
m_{3} & m_{4} & m_{34}
\end{array}\right)\left(\begin{array}{ccc}
j_{13} & j_{24} & j \\
m_{13} & m_{24} & m
\end{array}\right),
\end{aligned}
$$


where symbols $m_{i}$ are the eigenvalues of the projections on the $z$-axis of the related angular momenta $\boldsymbol{j}_{i}$.

The 9- $j$ symbol is invariant under an even permutation of rows or columns but is multiplied by $(-1)^{\left(j_{1}+j_{2}+j_{3}+j_{4}+j_{12}+j_{13}+j_{24}+j_{34}+j\right)}$, under an odd permutation of rows or columns, and under transposing operation. 


\section{A.6 List of analyzed reconstructions}

\begin{tabular}{|c|c|c|c|}
\hline Notation & Type of reconstruction & $\begin{array}{l}\text { Regularization } \\
\text { parameter, } \mu\end{array}$ & $\begin{array}{l}\text { Number of } \\
\text { iterations }\end{array}$ \\
\hline $\mathrm{Z} 1$ & $\begin{array}{l}\text { Reconstruction based on the Zeeman-effect data } \\
\text { and optimized for the whole corona. The poten- } \\
\text { tial field approximation is used as an initial con- } \\
\text { dition. }\end{array}$ & $10^{-3}$ & 47 \\
\hline $\mathrm{Z} 2$ & $\begin{array}{l}\text { Reconstruction based on the Zeeman-effect data } \\
\text { and optimized for the perturbed region only. The } \\
\text { potential field approximation is used as initial } \\
\text { condition. }\end{array}$ & $10^{-3}$ & 99 \\
\hline $\mathrm{H} 1$ & $\begin{array}{l}\text { Reconstruction based on the Hanle-effect data } \\
\text { and optimized for the whole corona. The poten- } \\
\text { tial field approximation is used as initial condi- } \\
\text { tion. }\end{array}$ & $10^{-5}$ & 7 \\
\hline $\mathrm{H} 2$ & $\begin{array}{l}\text { Reconstruction based on the Hanle-effect data } \\
\text { and optimized for the perturbed region only. The } \\
\text { potential field approximation is used as initial } \\
\text { condition. }\end{array}$ & $10^{-5}$ & 20 \\
\hline $\mathrm{H} 3$ & $\begin{array}{l}\text { Reconstruction based on the Hanle-effect data } \\
\text { and optimized for the perturbed region only. The } \\
\text { potential field approximation is used as initial } \\
\text { condition. }\end{array}$ & $10^{-5}$ & 59 \\
\hline ZH1 & $\begin{array}{l}\text { Reconstruction based on the Hanle-effect data } \\
\text { and optimized for the whole corona. The Z1- } \\
\text { solution is used as initial condition. }\end{array}$ & $10^{-5}$ & 5 \\
\hline $\mathrm{ZH} 2$ & $\begin{array}{l}\text { Reconstruction based on the Hanle-effect data } \\
\text { and optimized for the perturbed region only. The } \\
\text { Z1-solution is used as initial condition. }\end{array}$ & $10^{-5}$ & 13 \\
\hline $\mathrm{ZH} 3$ & $\begin{array}{l}\text { Reconstruction based on the Hanle-effect data } \\
\text { and optimized for the perturbed region only. The } \\
\text { Z1-solution is used as initial condition. }\end{array}$ & $10^{-5}$ & 30 \\
\hline
\end{tabular}

Table A.2: Notations for the performed reconstructions 


\section{A.7 Cross sections of the reconstructed field}

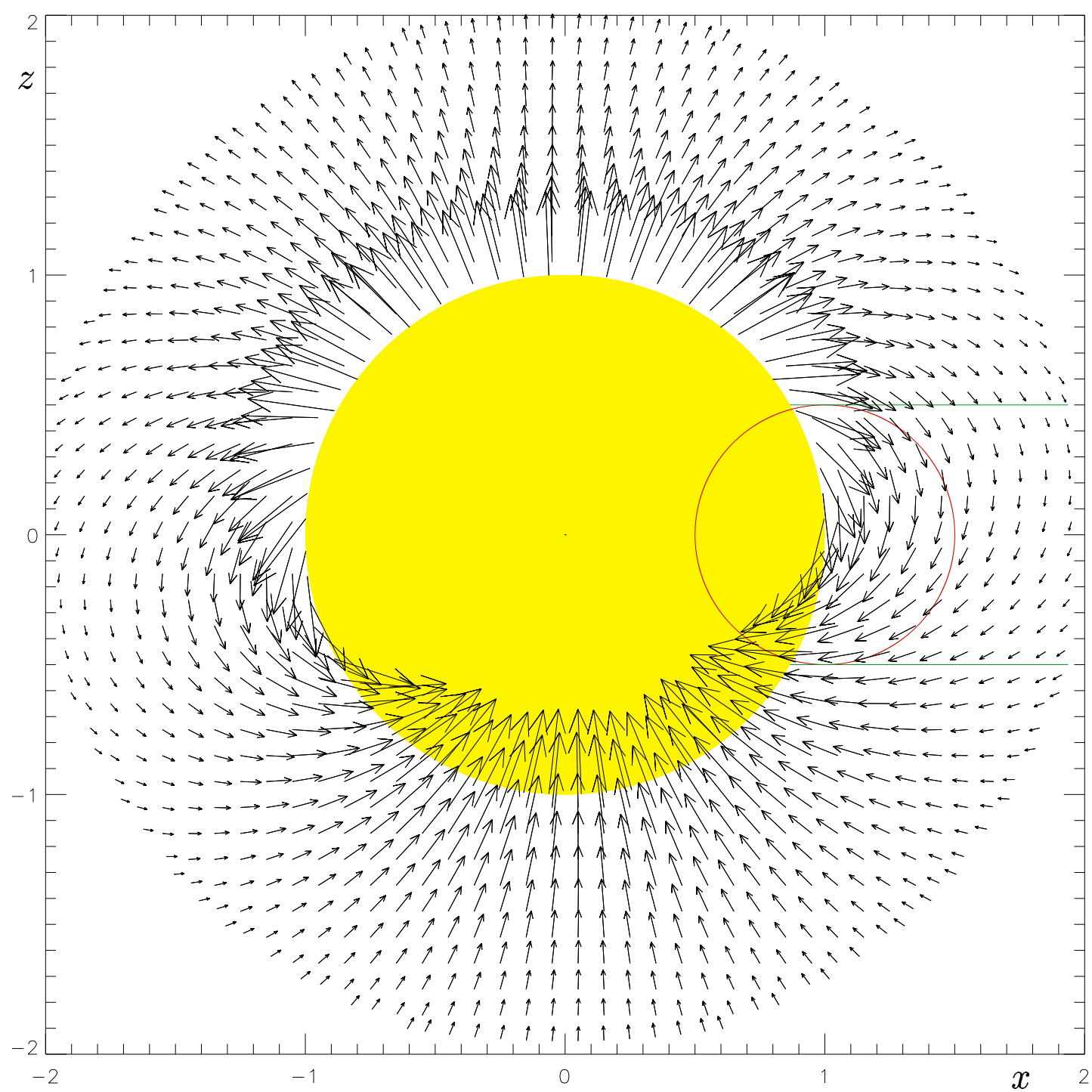

Figure A.2: (a) The model field configuration: the cross section defined by the $y=$ $-0.05 R_{\odot}$ plane. The red circle represents the projection of the perturbing circular current on the section plane. It should be noted that the current lies in the plane $y=0$. The green horizontal lines bound the analyzed perturbed region. 


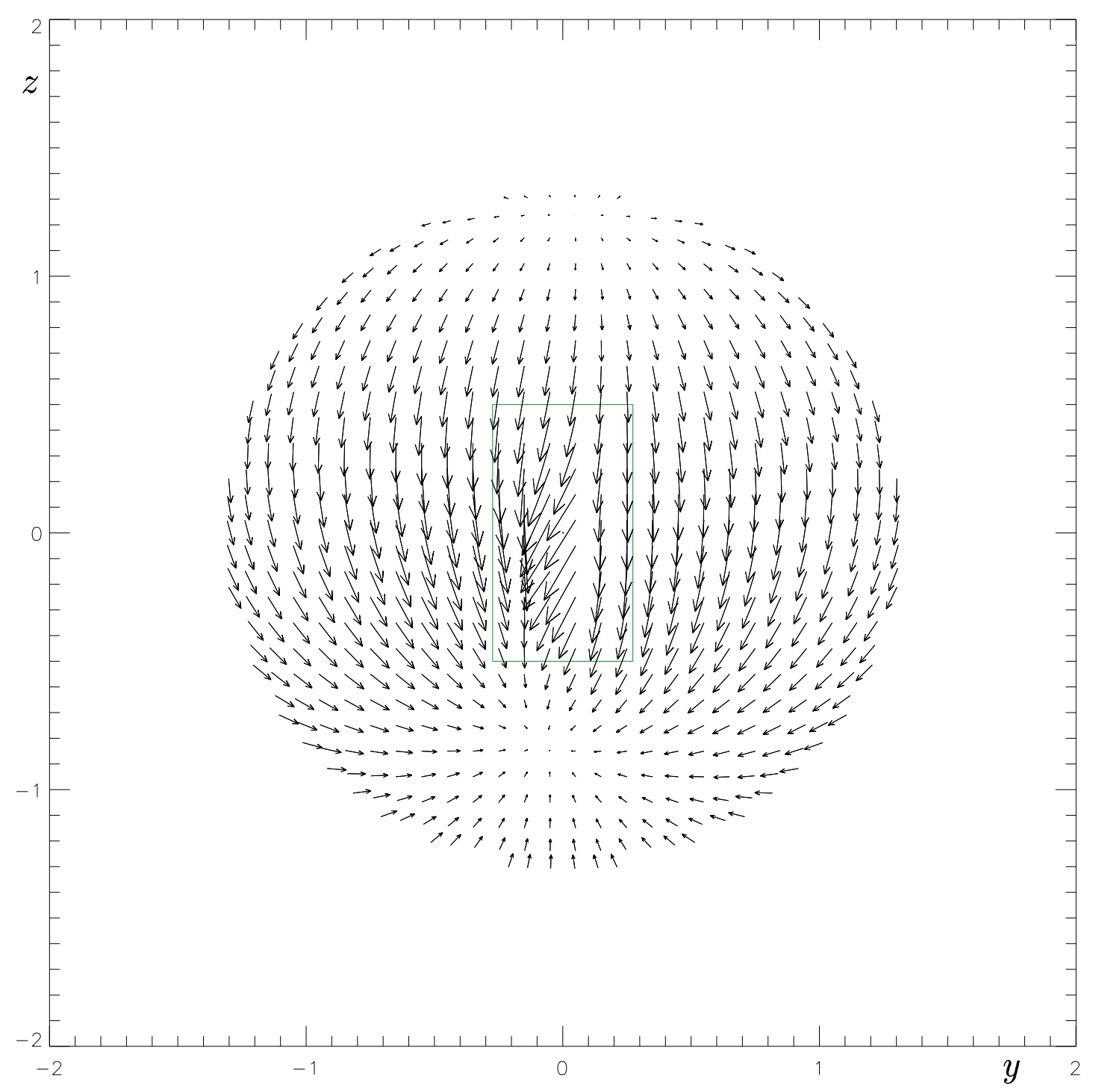

Figure A.2: $(b)$ The model field configuration: the cross section defined by the $x=$ $1.55 R_{\odot}$ plane. The green box bounds the analyzed perturbed region. 


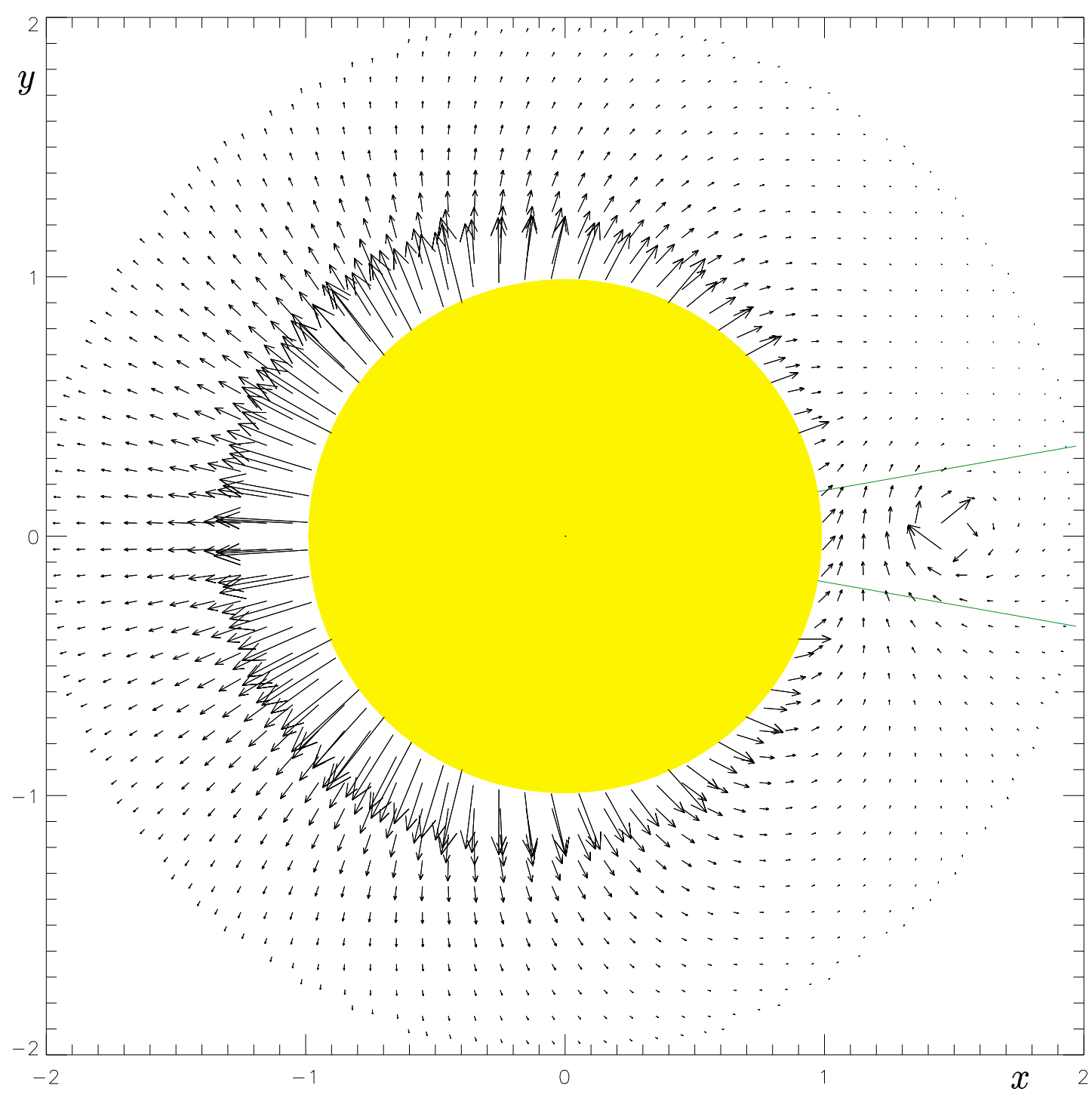

Figure A.2: The model field configuration: the cross section defined by the $z=0.15 R_{\odot}$ plane. The two green lines bound the analyzed perturbed region. 


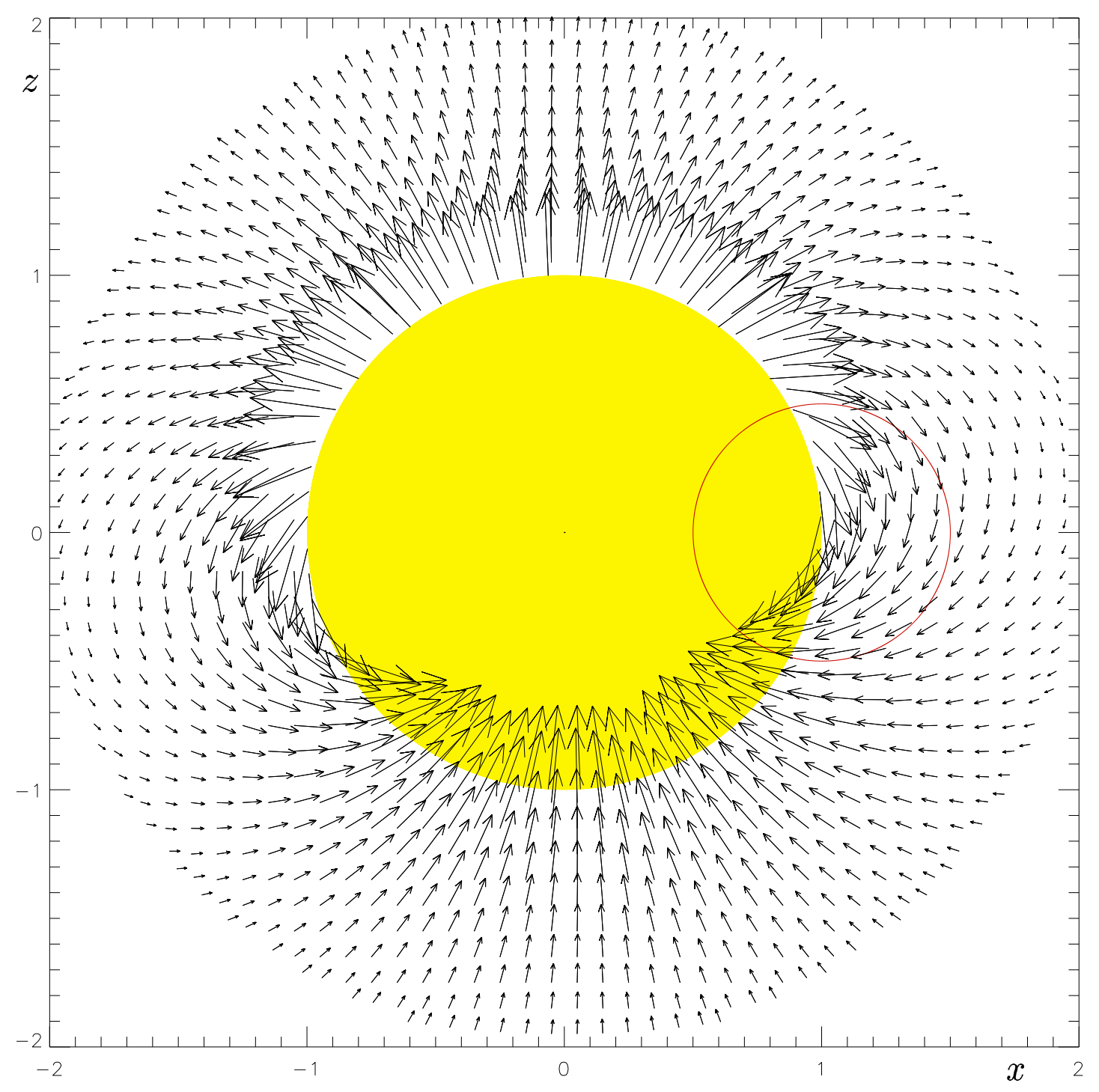

Figure A.3: (a) The potential field reconstruction: the cross section defined by the $y=-0.05 R_{\odot}$ plane. 


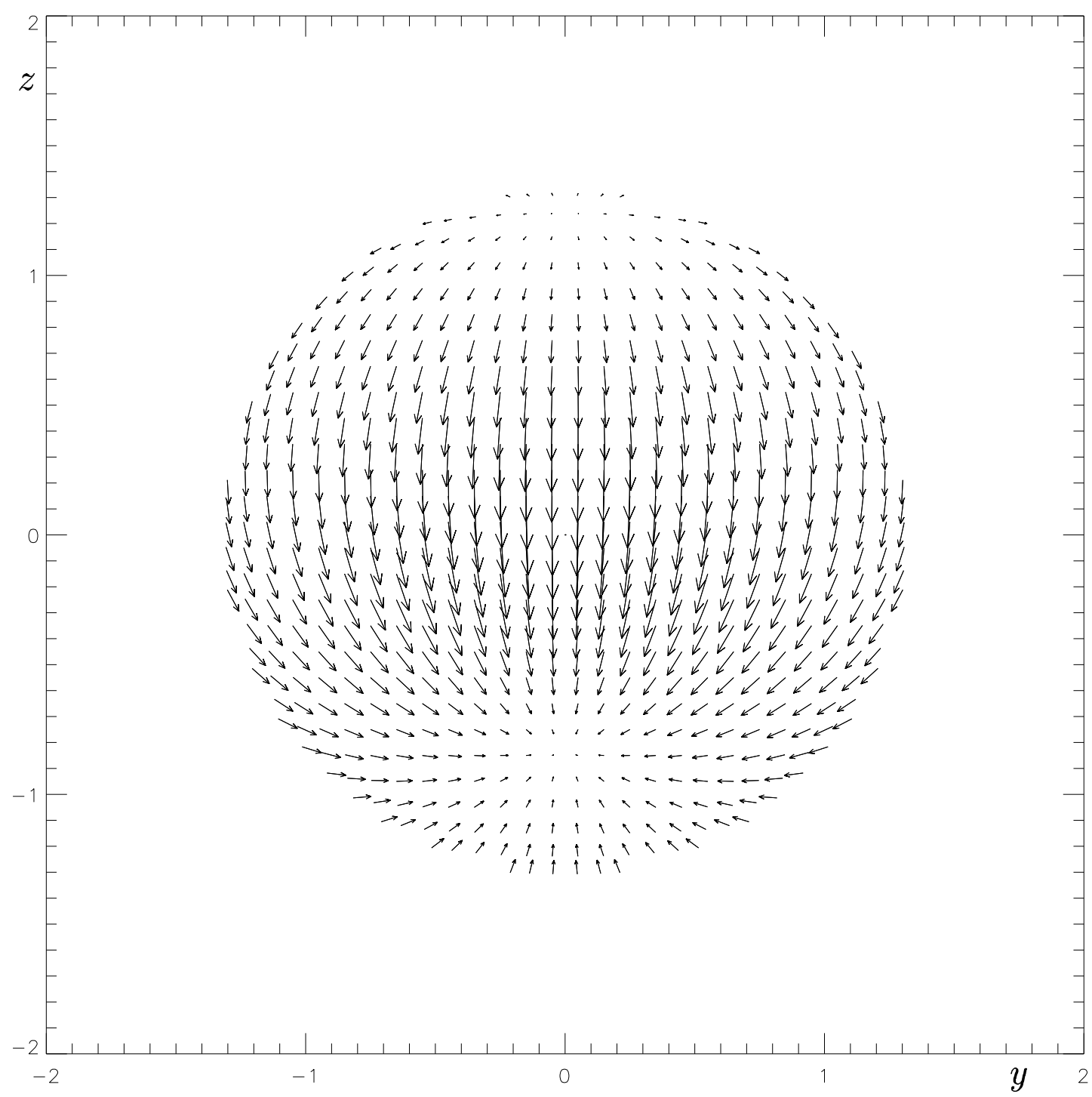

Figure A.3: $(b)$ The potential field reconstruction: the cross section defined by the $x=1.55 R_{\odot}$ plane. 


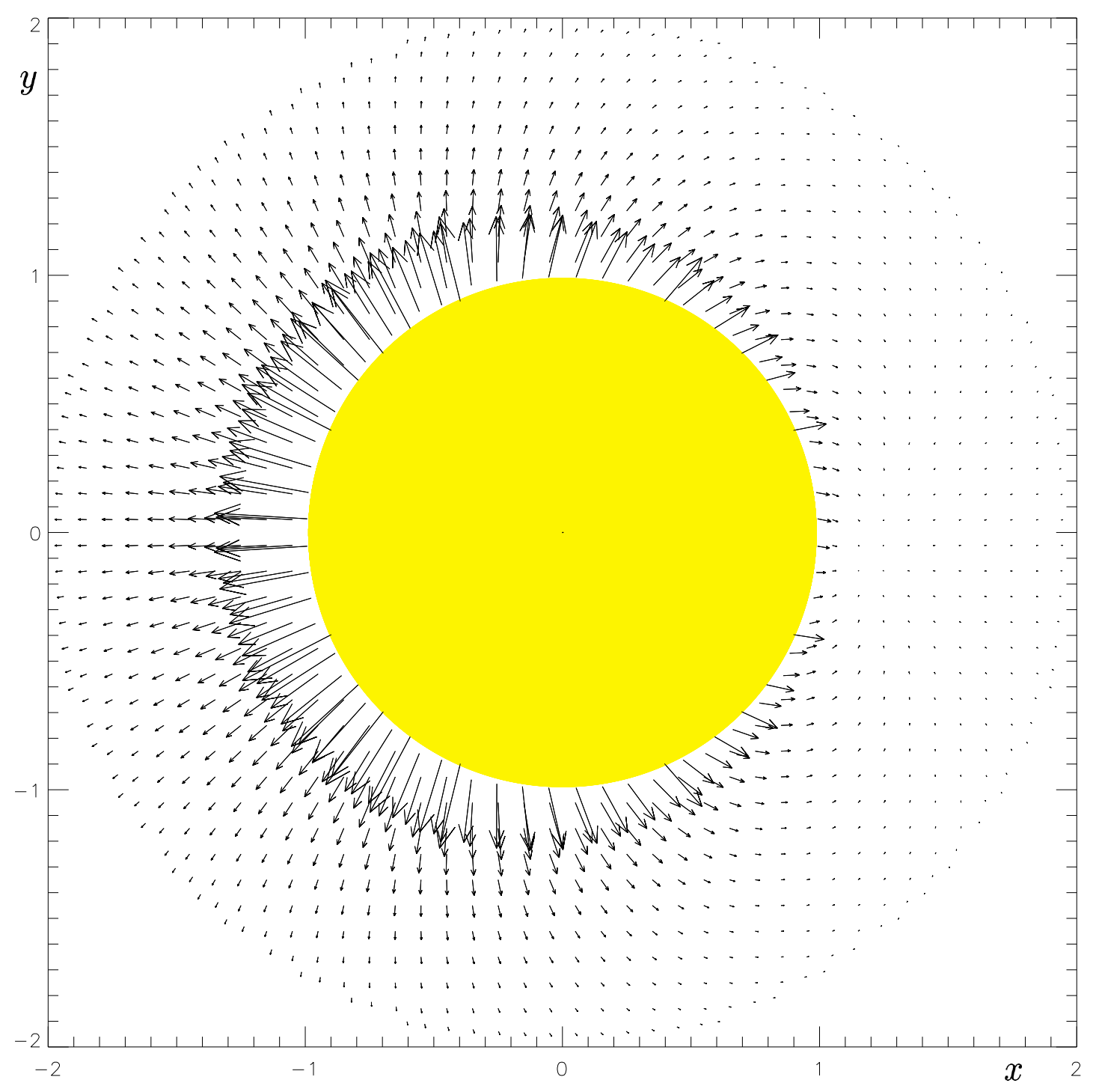

Figure A.3: The potential field reconstruction: the cross section defined by the $z=$ $0.15 R_{\odot}$ plane. 


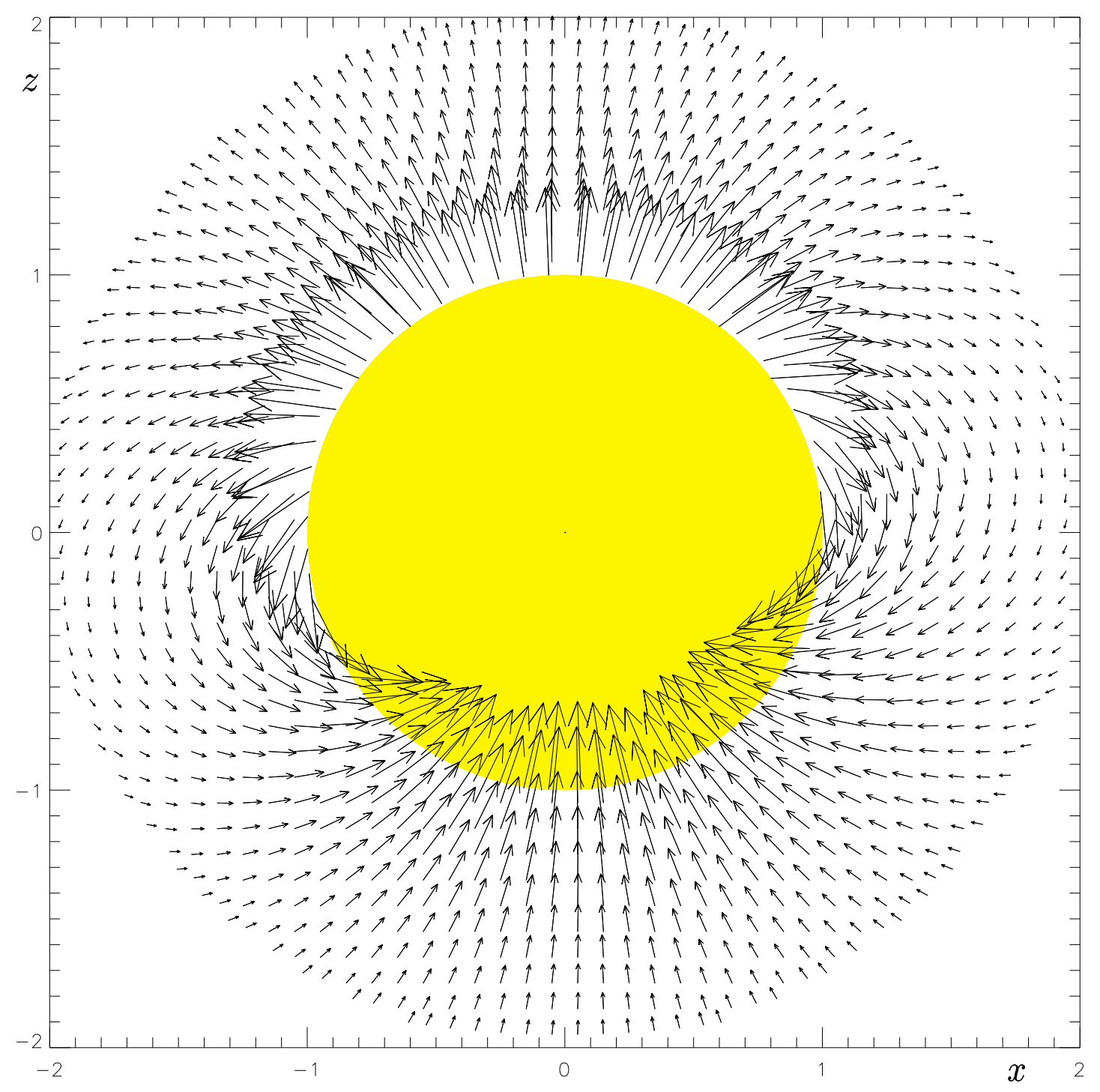

Figure A.4: (a) The Zeeman-effect reconstruction for $\mu=10^{-3}$ after 47 iteration (Z1): the cross section defined by the $y=-0.05 R_{\odot}$ plane. 


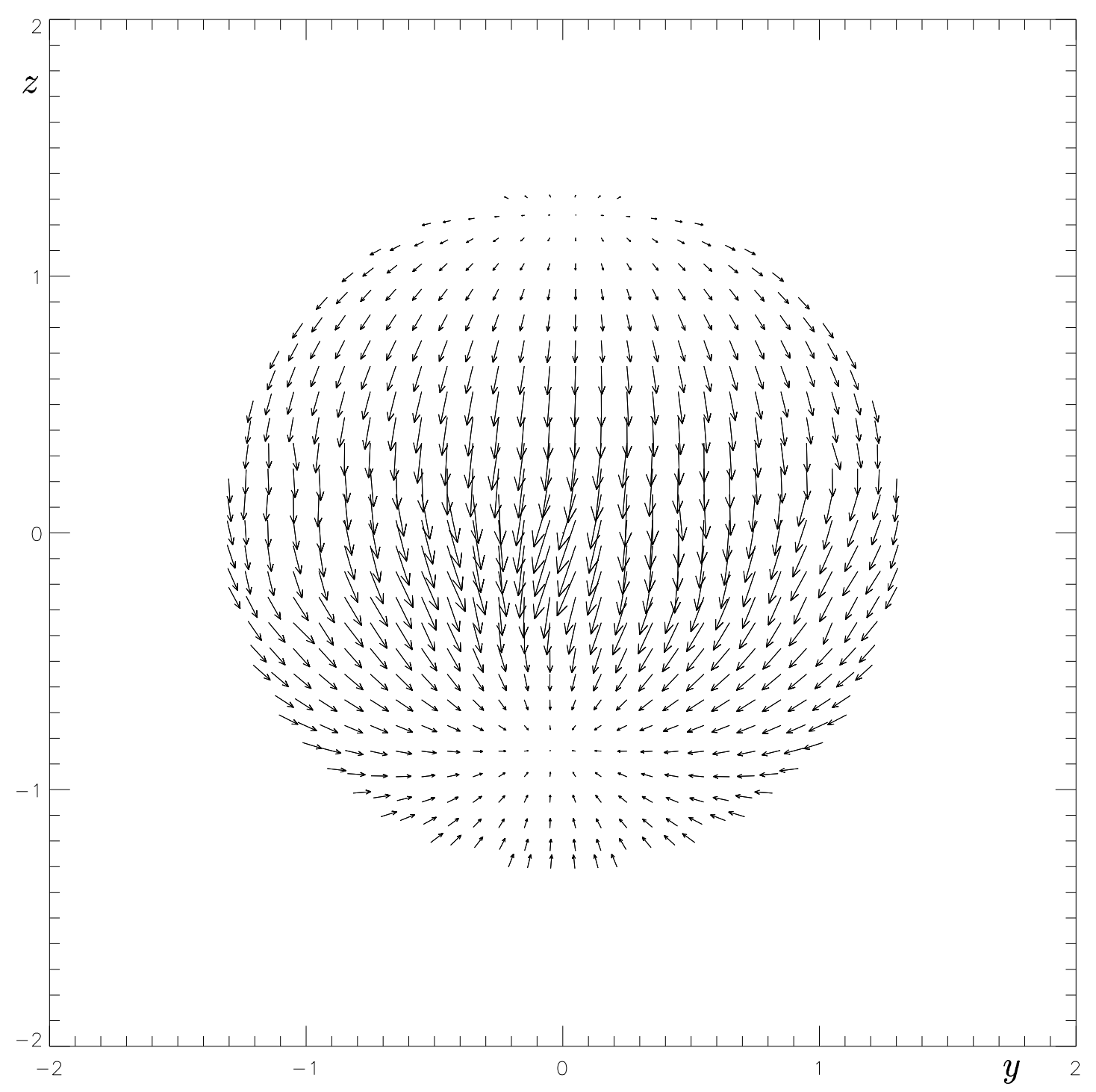

Figure A.4: $(b)$ The Zeeman-effect reconstruction for $\mu=10^{-3}$ after 47 iteration (Z1): the cross section defined by the $x=1.55 R_{\odot}$ plane. 


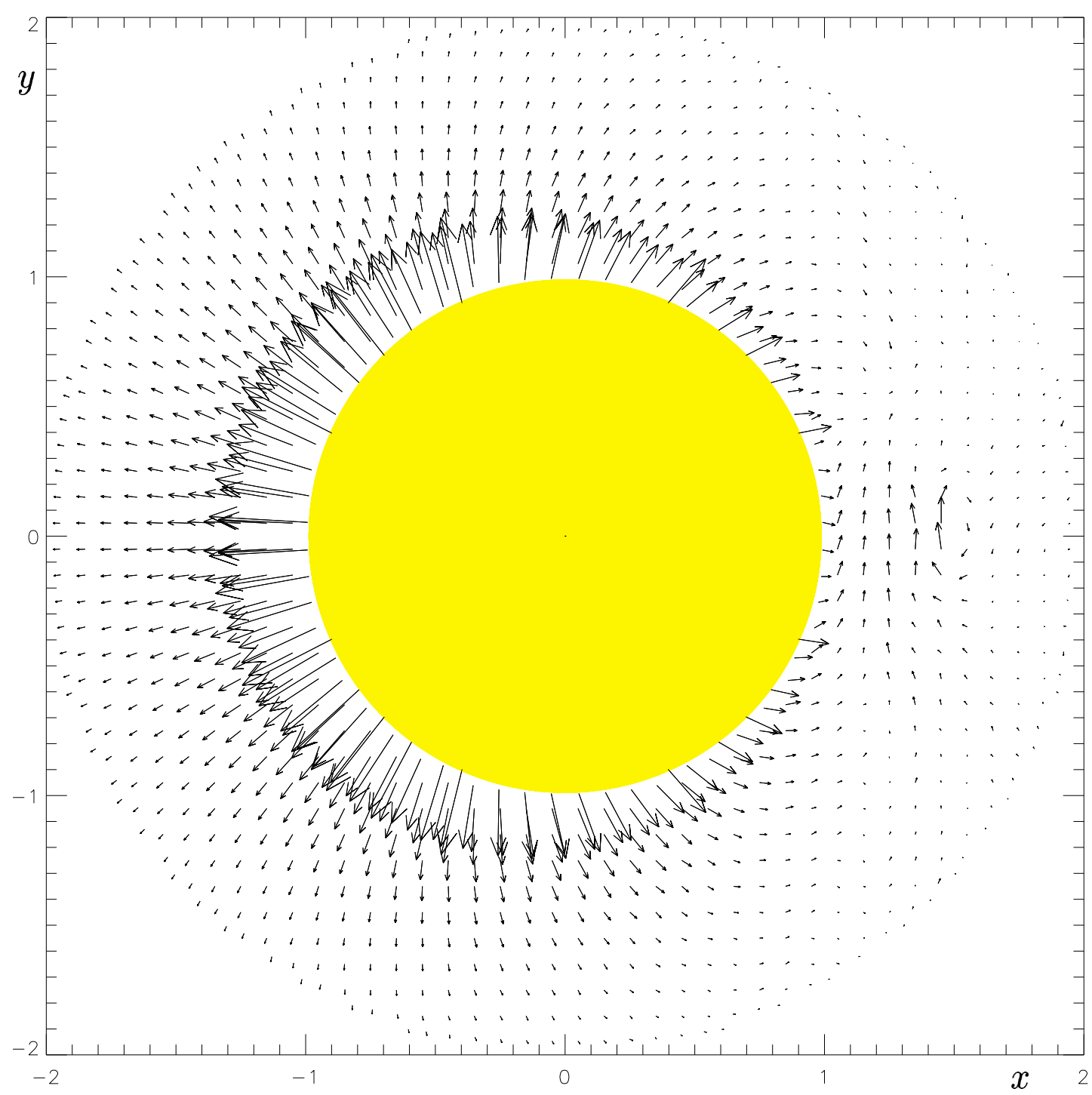

Figure A.4: $(c)$ The Zeeman-effect reconstruction for $\mu=10^{-3}$ after 47 iteration (Z1): the cross section defined by the $z=0.15 R_{\odot}$ plane. 


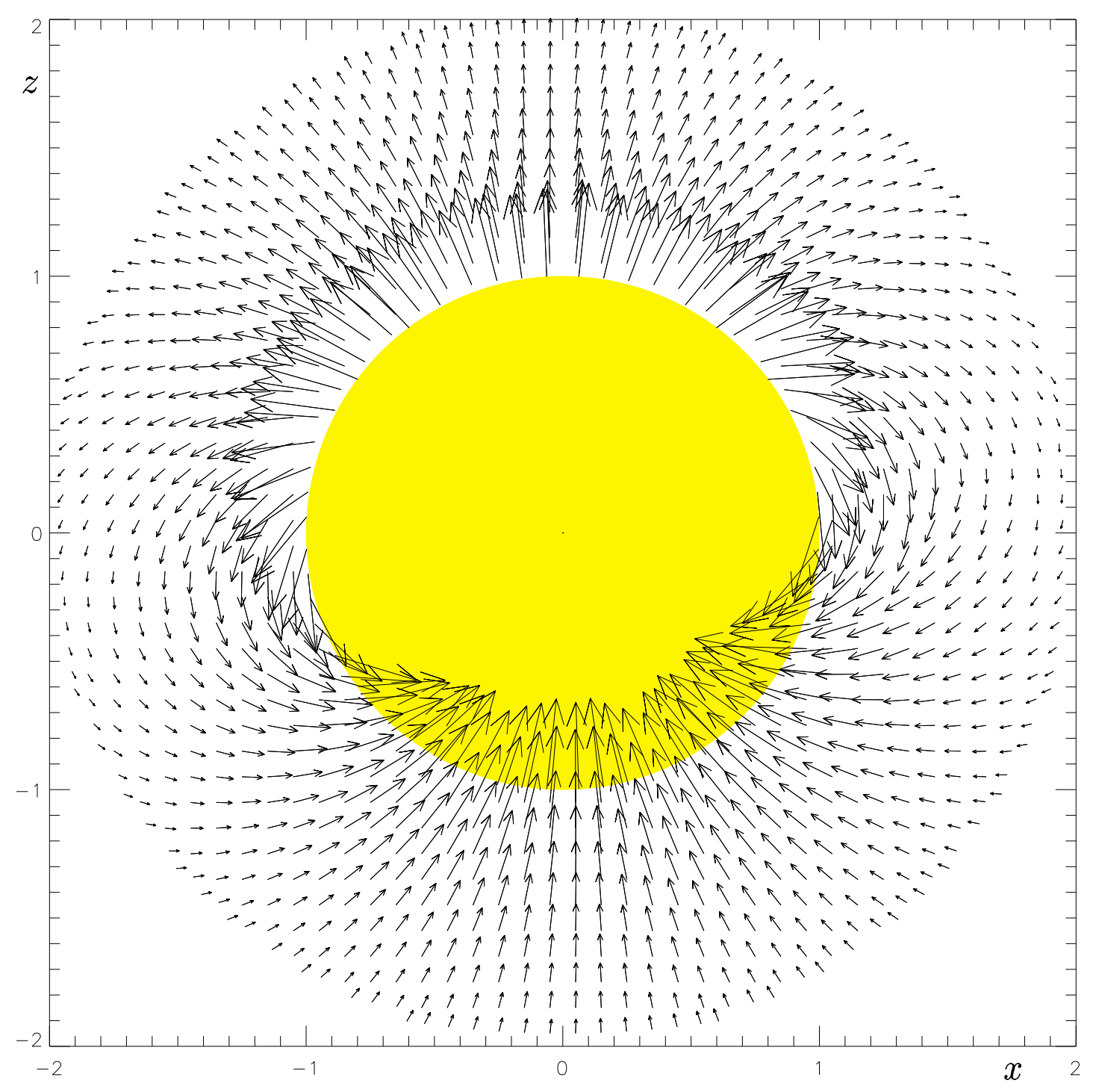

Figure A.5: (a) The Zeeman-effect reconstruction for $\mu=10^{-3}$ after 99 iteration (Z2): the cross section defined by the $y=-0.05 R_{\odot}$ plane. 


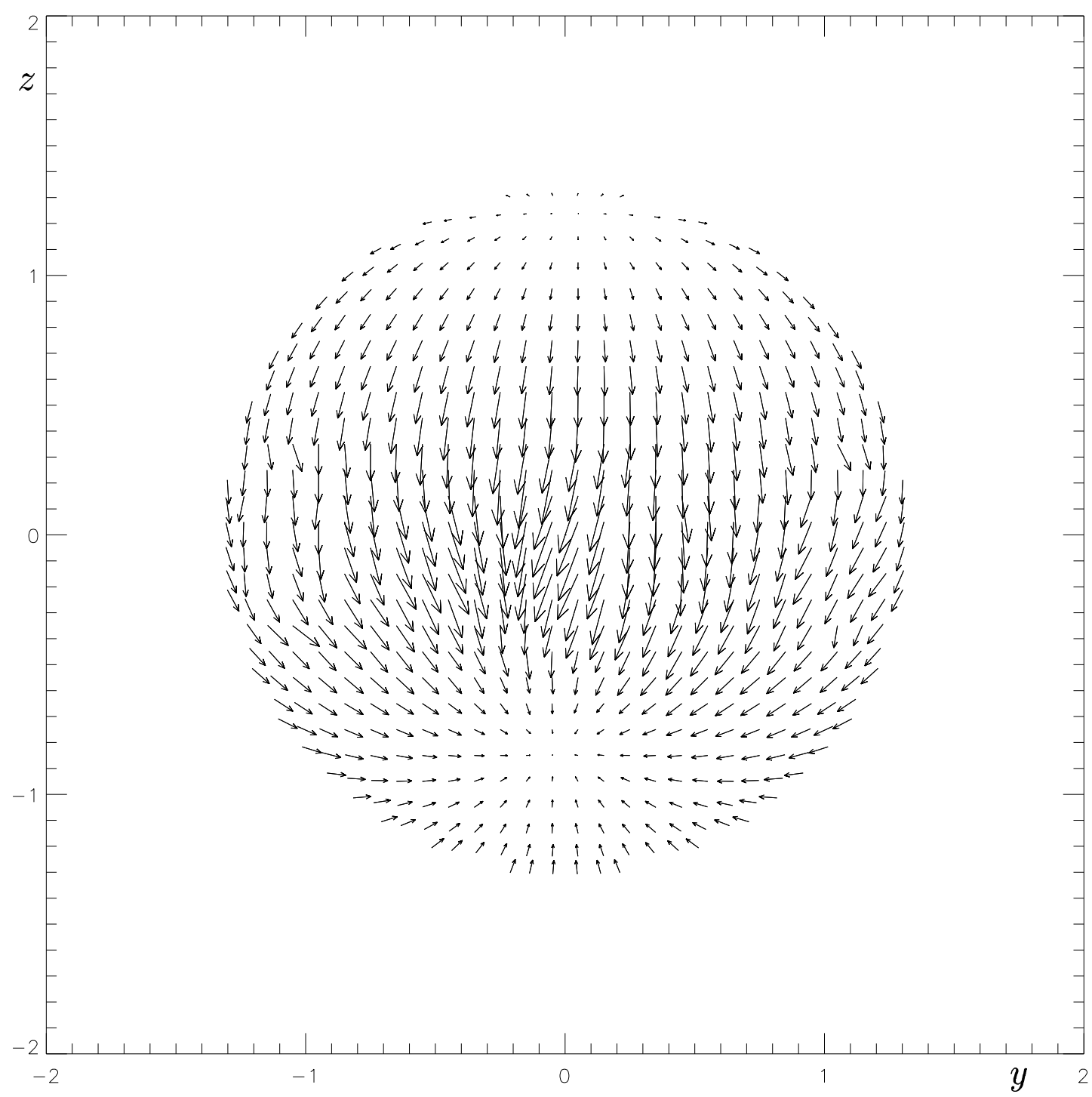

Figure A.5: $(b)$ The Zeeman-effect reconstruction for $\mu=10^{-3}$ after 99 iteration (Z2): the cross section defined by the $x=1.55 R_{\odot}$ plane. 


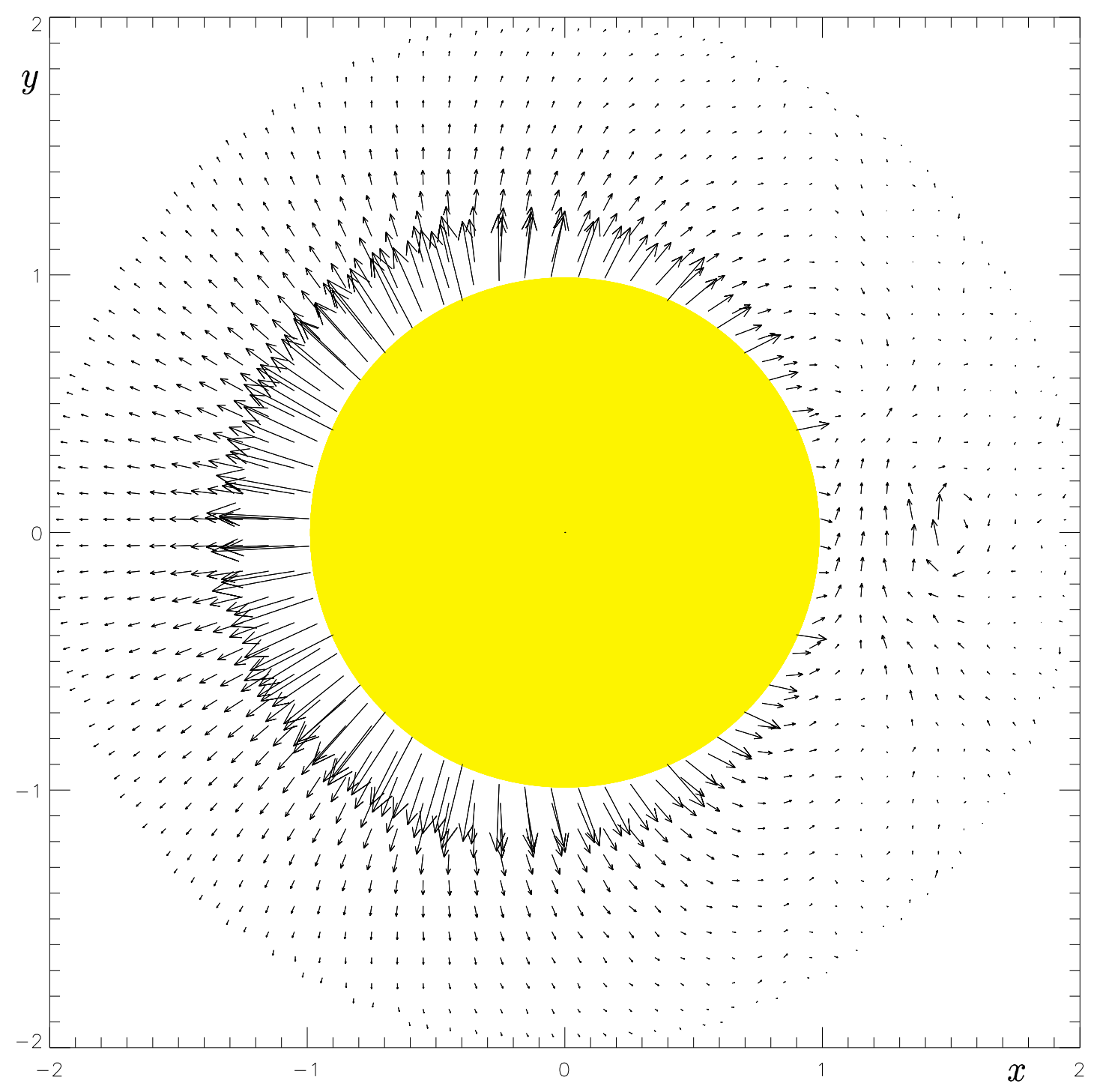

Figure A.5: (c) The Zeeman-effect reconstruction for $\mu=10^{-3}$ after 99 iteration (Z2): the cross section defined by the $z=0.15 R_{\odot}$ plane. 


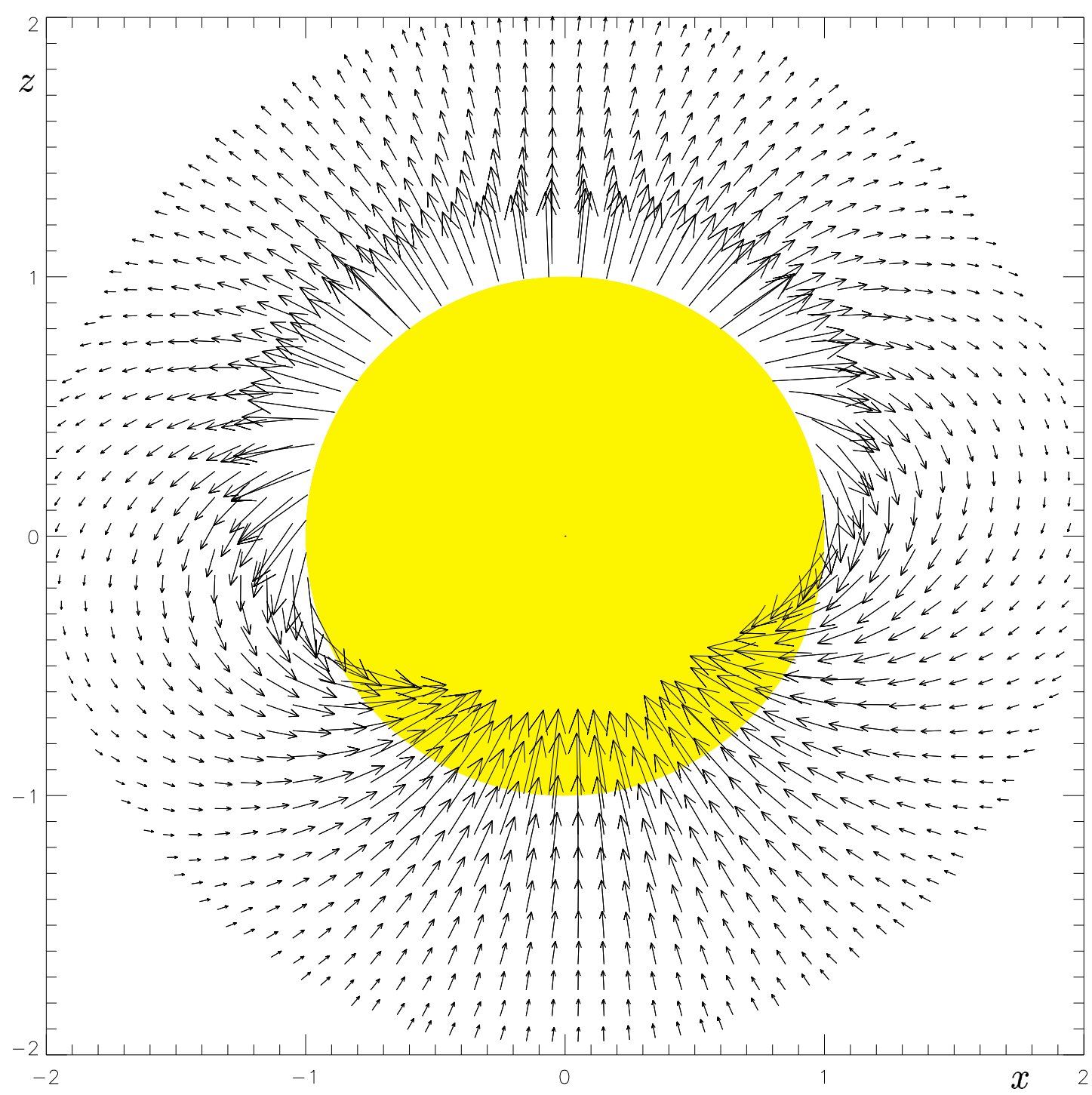

Figure A.6: (c) The Hanle-effect reconstruction for $\mu=10^{-5}$ after 7 iteration (H1): the cross section defined by the $y=-0.05 R_{\odot}$ plane. 


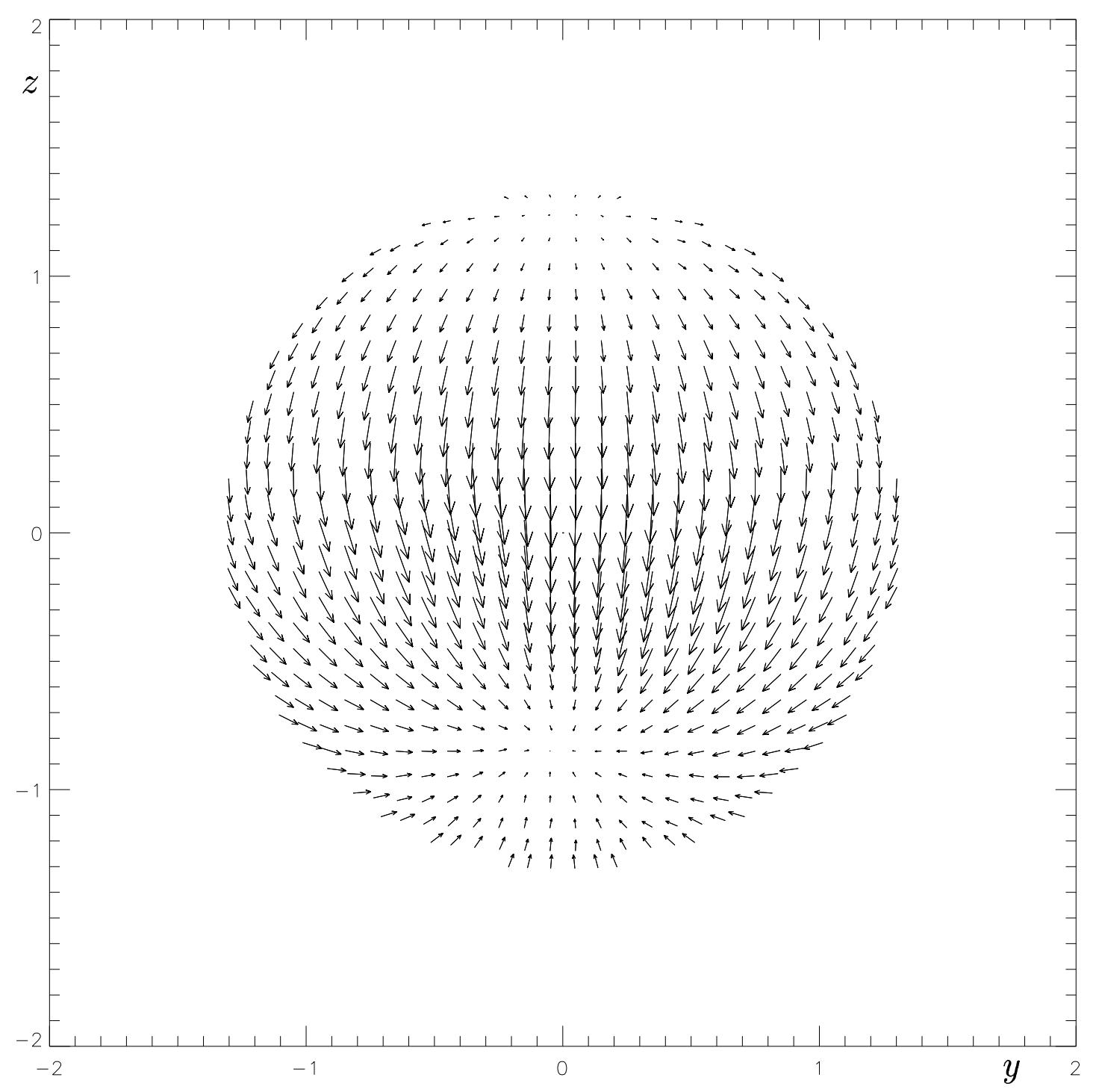

Figure A.6: (b) The Hanle-effect reconstruction for $\mu=10^{-5}$ after 7 iteration (H1): the cross section defined by the $x=1.55 R_{\odot}$ plane. 


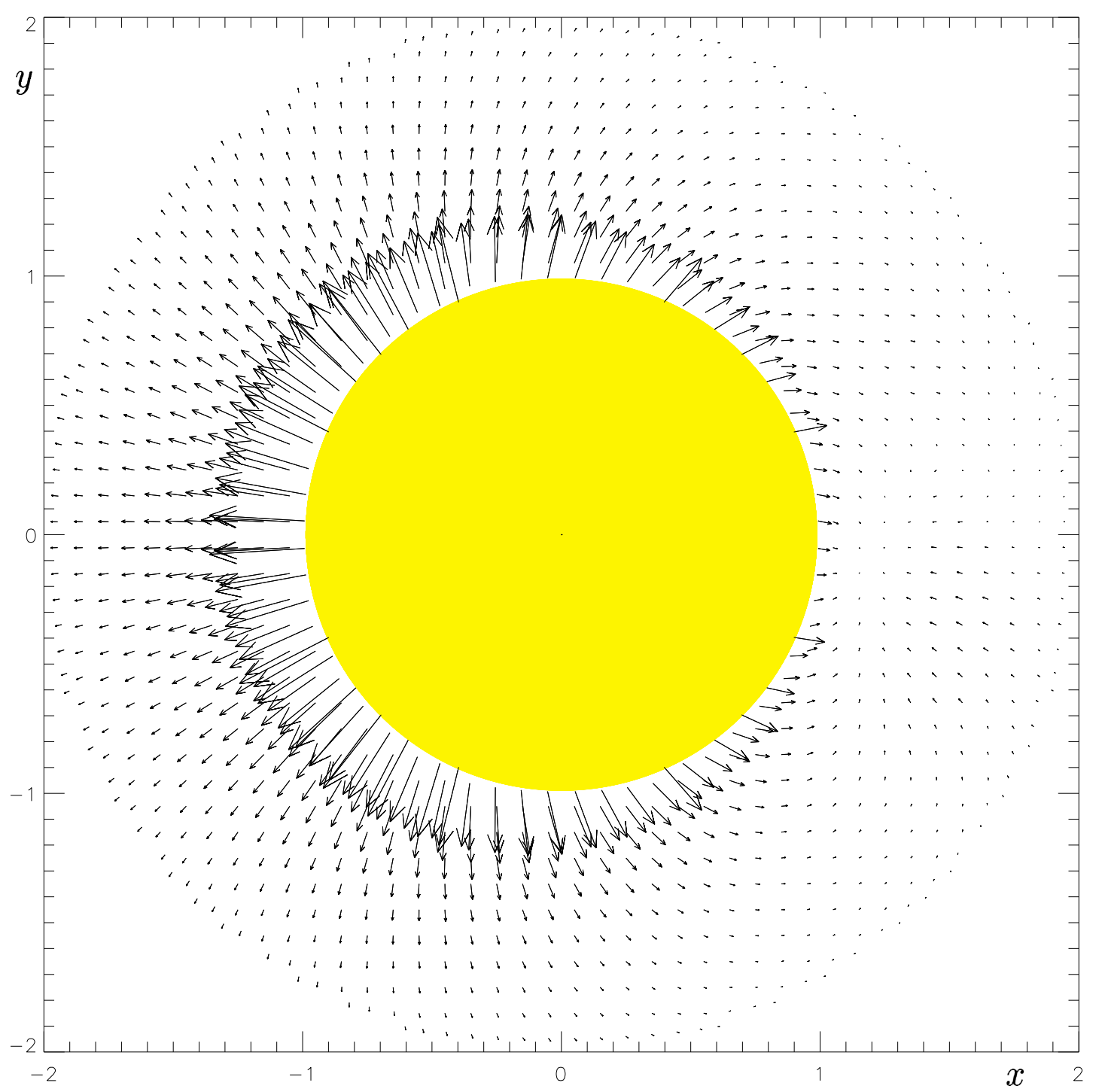

Figure A.6: (c) The Hanle-effect reconstruction for $\mu=10^{-5}$ after 7 iteration (H1): the cross section defined by the $z=0.15 R_{\odot}$ plane. 


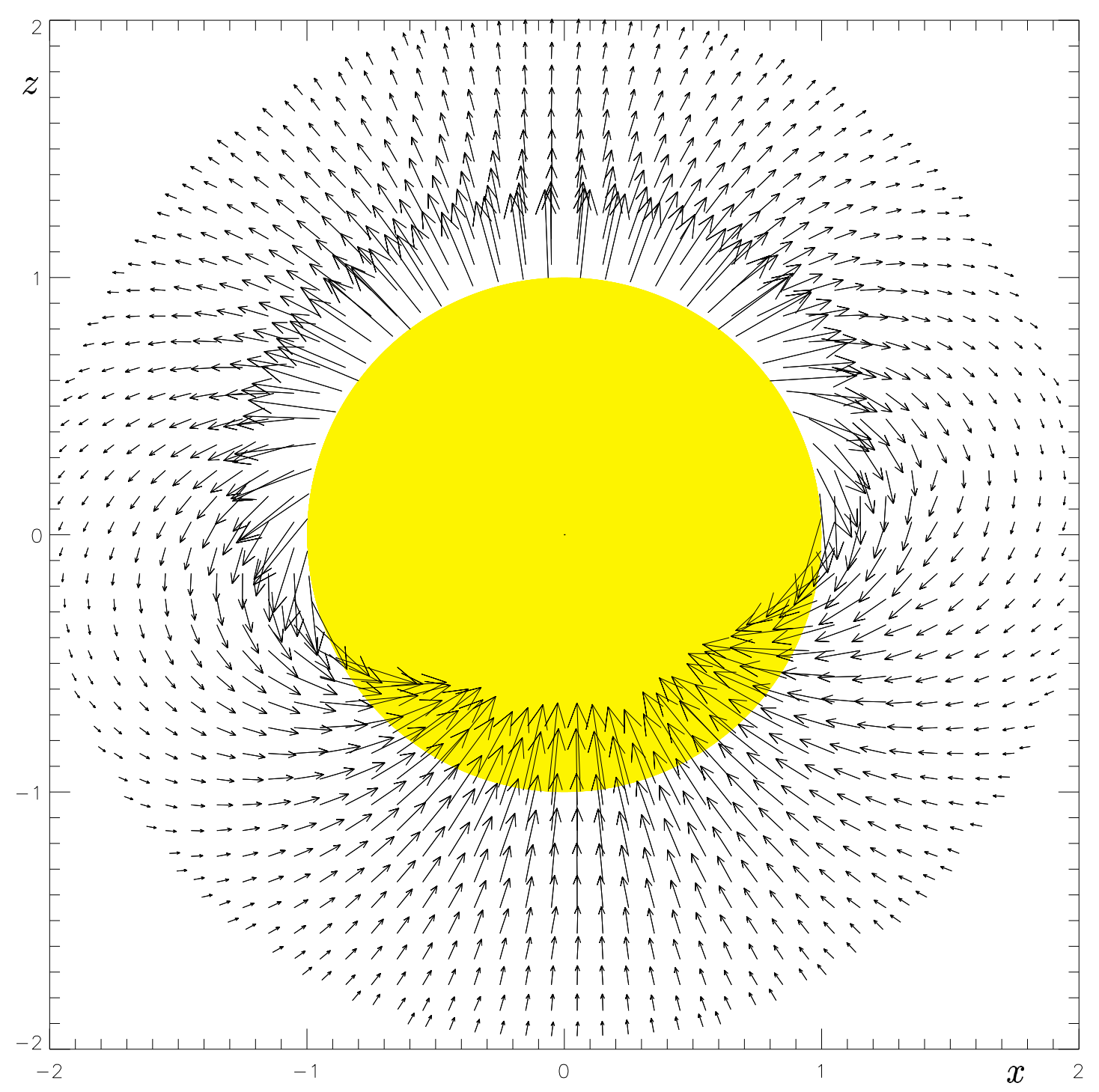

Figure A.7: (a) The Hanle-effect reconstruction for $\mu=10^{-5}$ after 20 iteration (H2): the cross section defined by the $y=-0.05 R_{\odot}$ plane. 


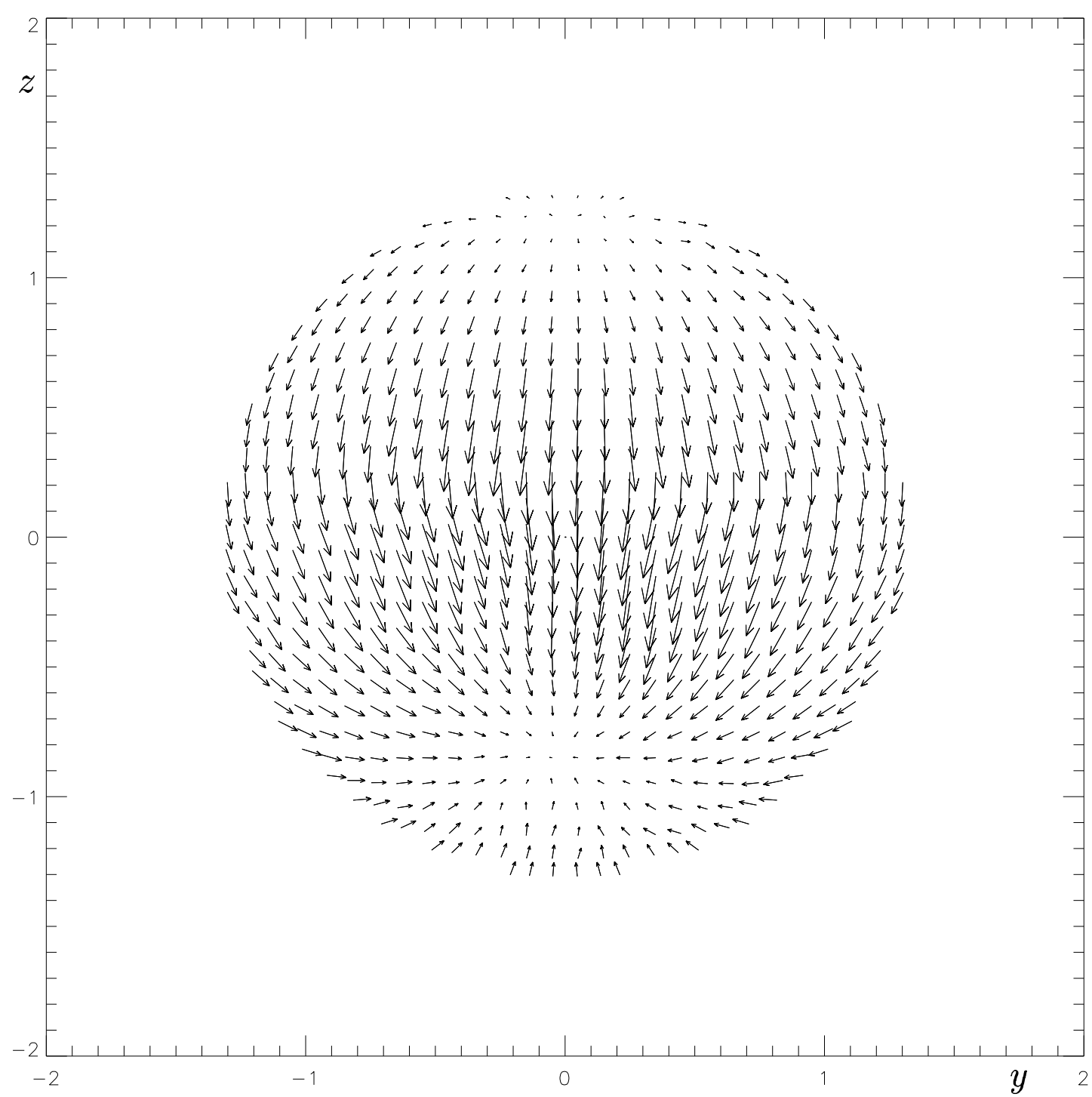

Figure A.7: (b) The Hanle-effect reconstruction for $\mu=10^{-5}$ after 20 iteration (H2): the cross section defined by the $x=1.55 R_{\odot}$ plane. 


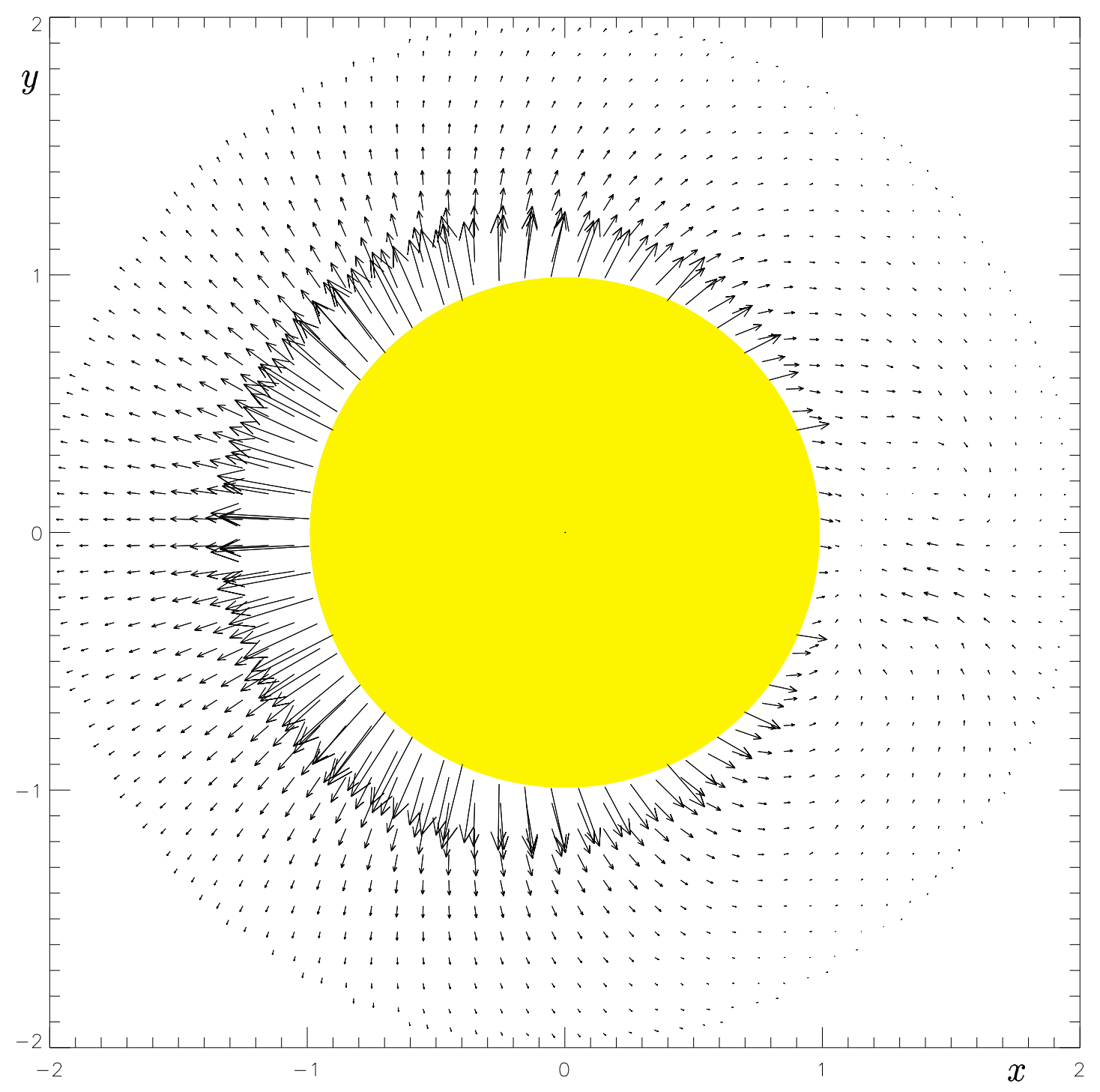

Figure A.7: (c) The Hanle-effect reconstruction for $\mu=10^{-5}$ after 20 iteration (H2): the cross section defined by the $z=0.15 R_{\odot}$ plane. 


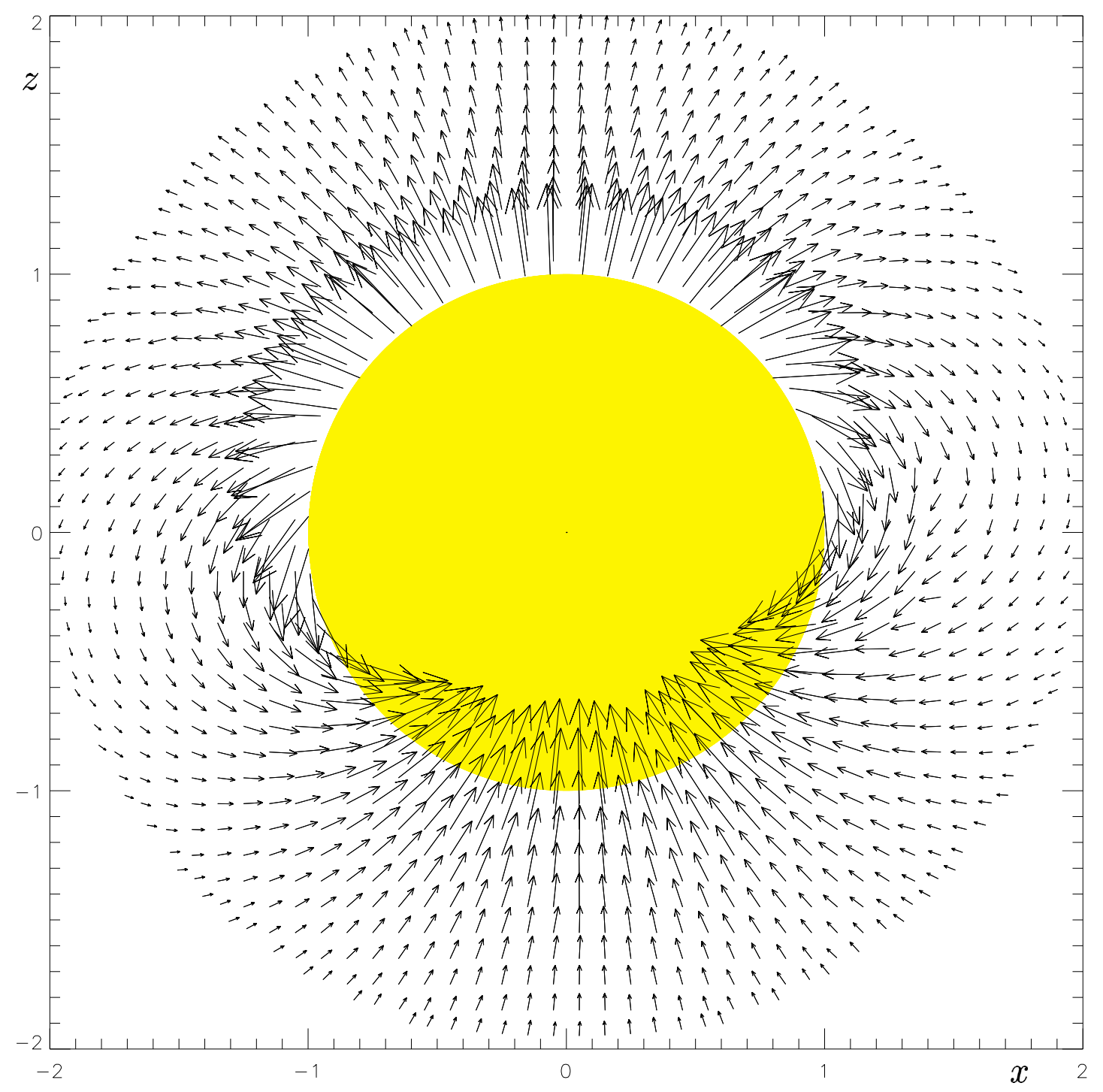

Figure A.8: (a) The Hanle-effect reconstruction for $\mu=10^{-5}$ after 59 iteration (H3): the cross section defined by the $y=-0.05 R_{\odot}$ plane. 


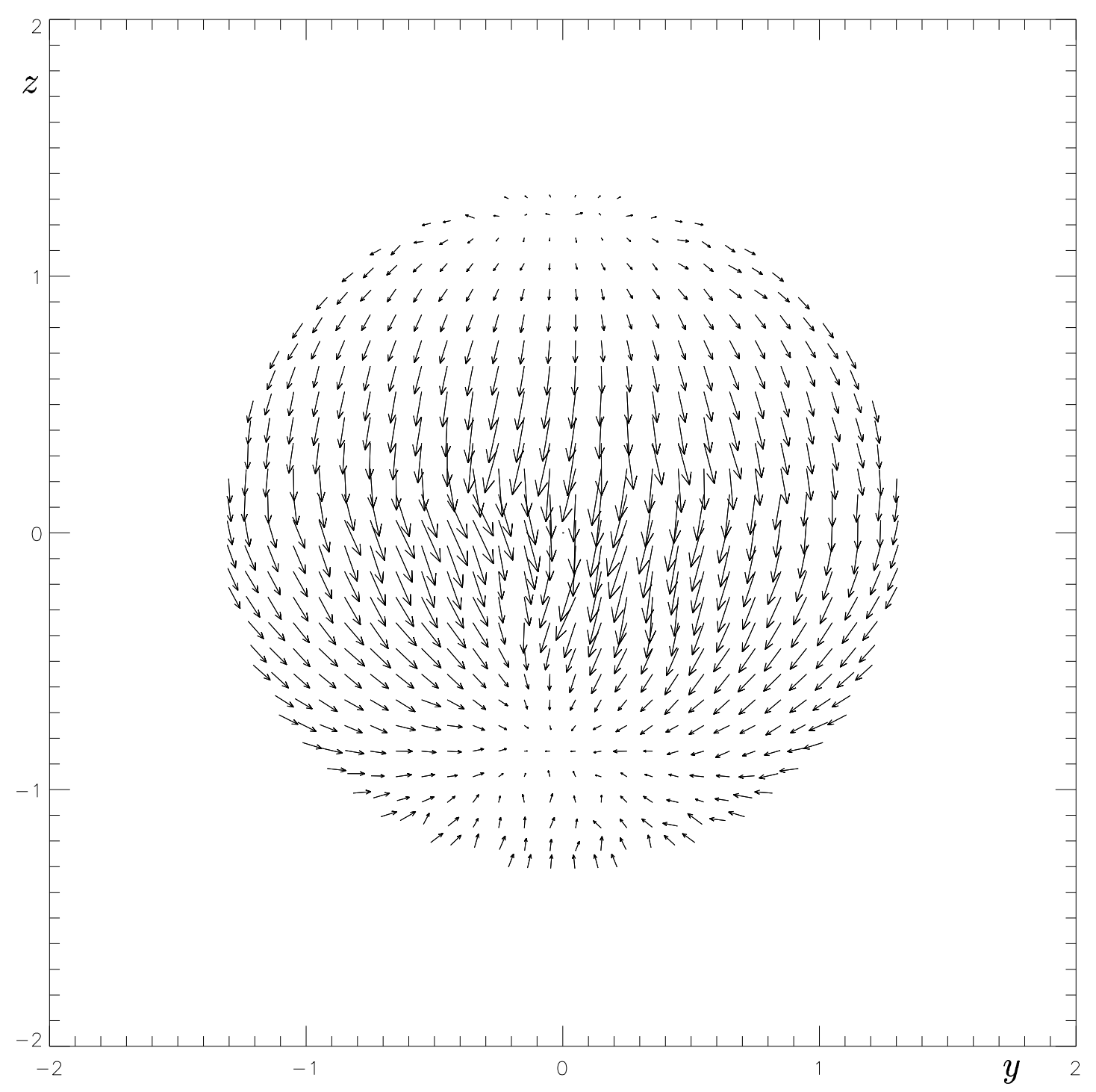

Figure A.8: $(b)$ The Hanle-effect reconstruction for $\mu=10^{-5}$ after 59 iteration (H3): the cross section defined by the $x=1.55 R_{\odot}$ plane. 


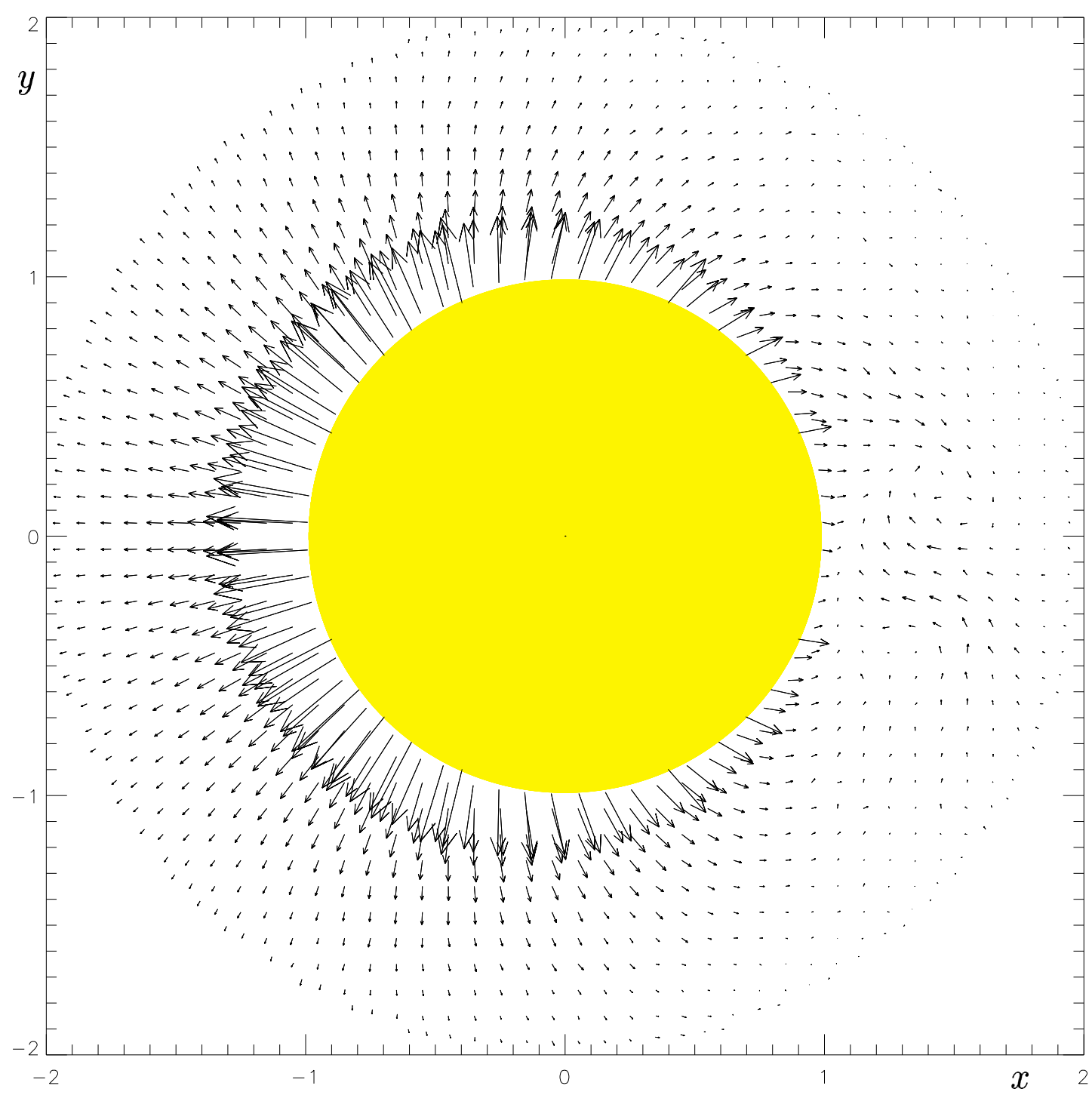

Figure A.8: (c) The Hanle-effect reconstruction for $\mu=10^{-5}$ after 59 iteration (H3): the cross section defined by the $z=0.15 R_{\odot}$ plane. 


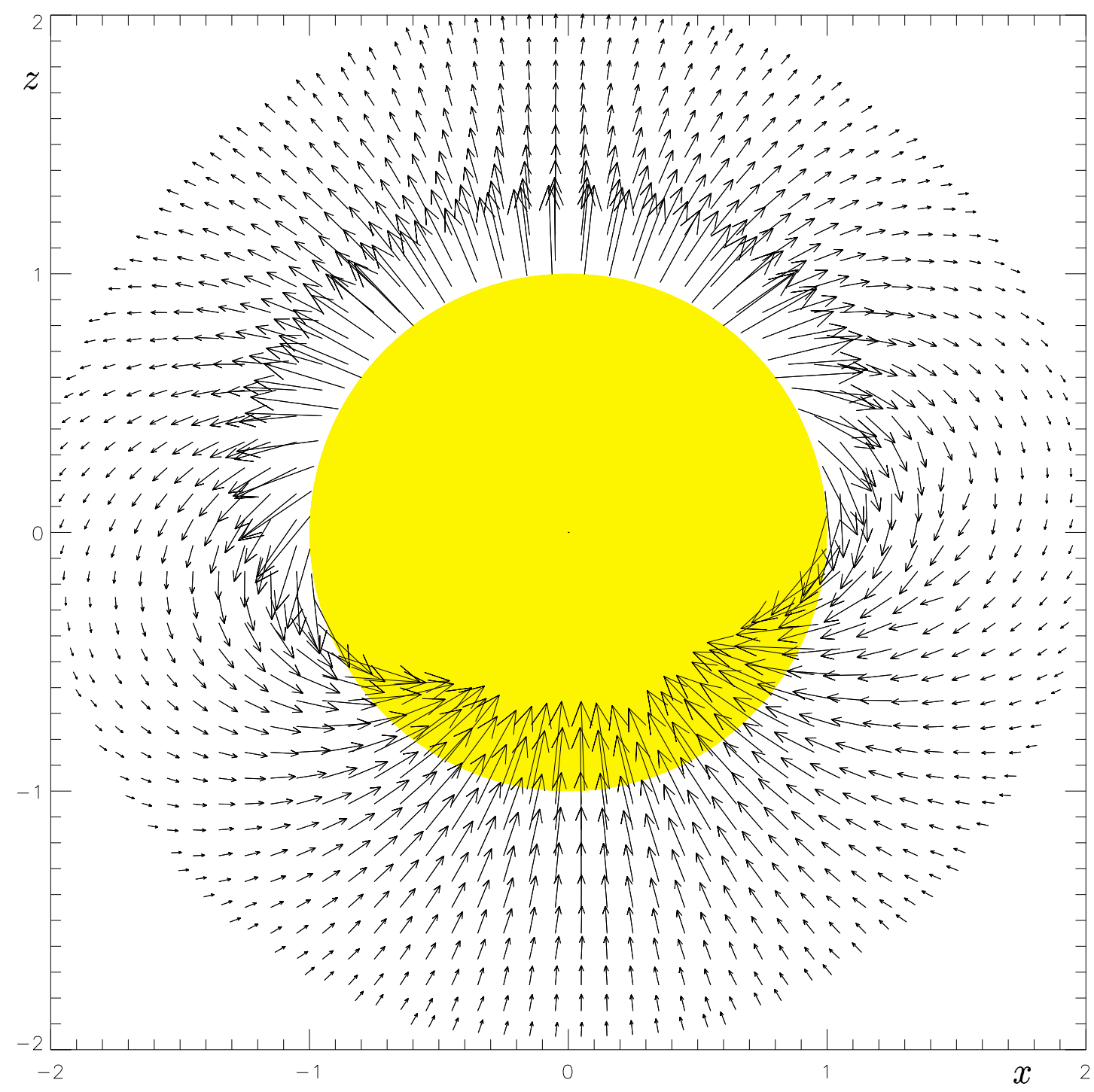

Figure A.9: (a) The Hanle-effect reconstruction for $\mu=10^{-5}$ after 13 iterations (ZH2). Here the Zeeman-effect solution Z1 depicted in the Figs. A.4 was used as initial field in the iterations. It is shown the cross section defined by the $y=-0.05 R_{\odot}$ plane. 


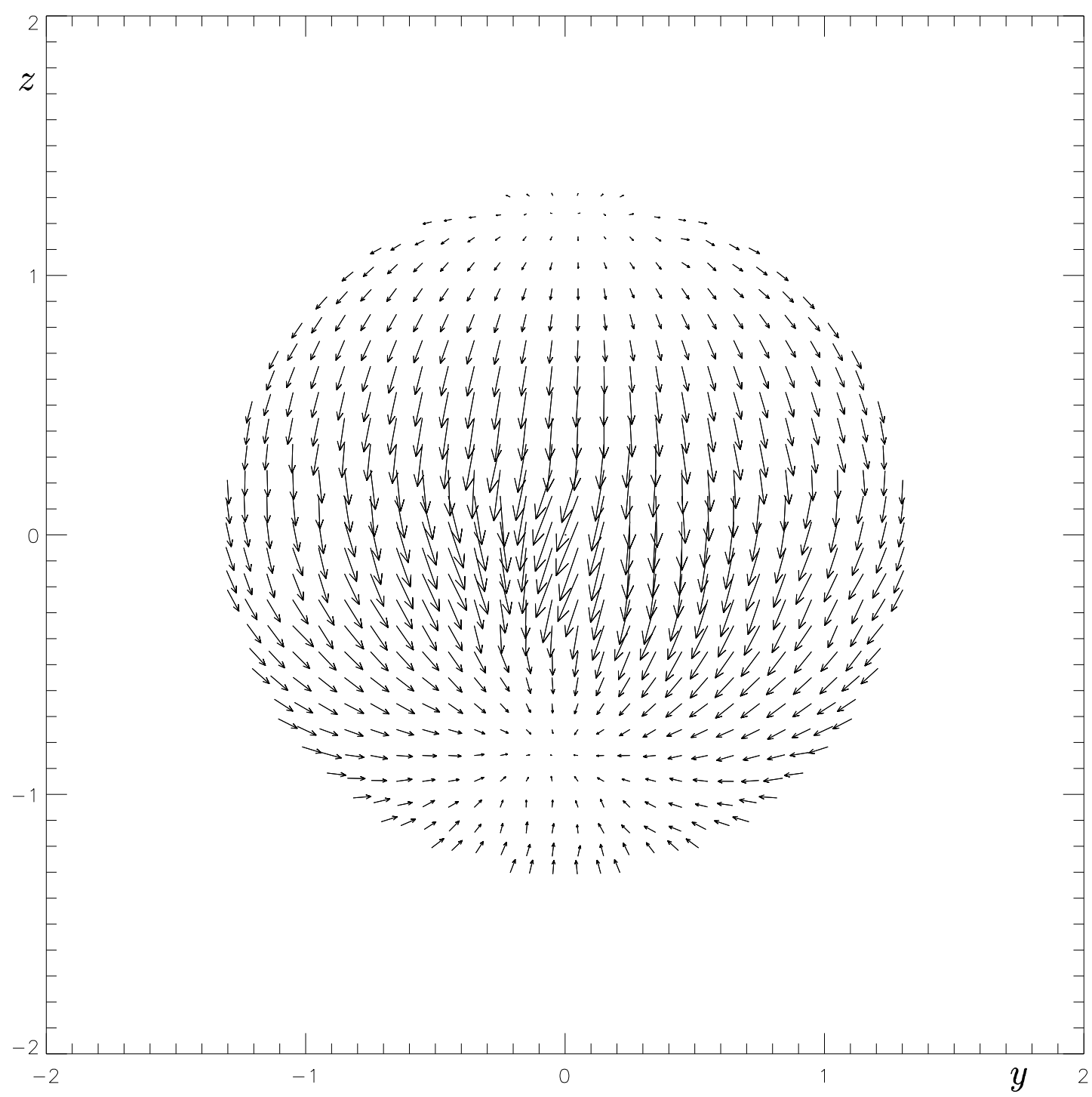

Figure A.9: (b) The Hanle-effect reconstruction for $\mu=10^{-5}$ after 13 iterations (ZH2). Here the Zeeman-effect solution Z1 depicted in the Figs. A.4 was used as initial field in the iterations. It is shown the cross section defined by the $x=1.55 R_{\odot}$ plane. 


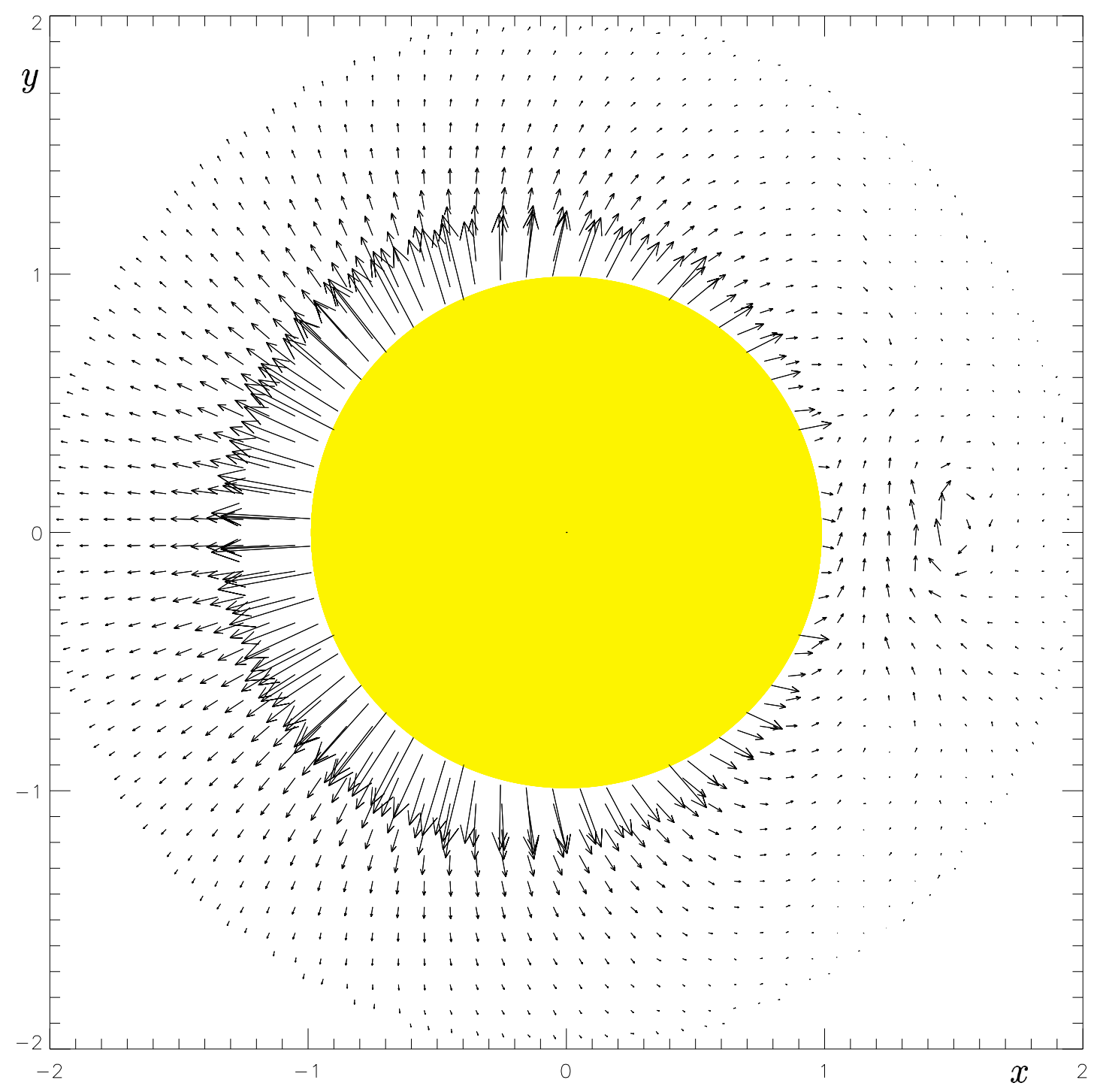

Figure A.9: (c) The Hanle-effect reconstruction for $\mu=10^{-5}$ after 13 iterations (ZH2). Here the Zeeman-effect solution Z1 depicted in the Figs. A.4 was used as initial field in the iterations. It is shown the cross section defined by the $z=0.15 R_{\odot}$ plane. 


\section{Bibliography}

Allen, C.W., 1973, Astrophysical Quantities, University of London, The Athlone Press, 3rd edition, 1973

Altschuler, M.D. \& Newkirk, G.Jr., 1969, Magnetic fields and the structure of the solar corona, Sol. Phys., 9, 131

Arnaud, J., 1982, Observed polarization of the Fe XIV 5303 coronal emission line, A\&A, 112,350

Arnaud, J., 1982, The analysis of Fe XIV coronal emission-line polarization measurements, A\&A, 116, 248

Arnaud, J., Newkirk, G.,Jr, 1987, Mean properties of the polarization of the Fe XIII $10747 \AA$ coronal emission line, A\&A, 178, 263

Bellan, P.M., 2000, Spheromaks: a practical application of magnetohydrodynamic dynamos and plasma self-organization, London: Imperial College Press, 2000

Boffin, H.M.J., Steeghs, D., Cuypers, J., (Eds.), 2001, Astrotomography: indirect imaging methods in observational astronomy, Springer, 2001

Brueckner, G.E., et al., 1995, The Large Angle Spectroscopic Coronagraph (LASCO), Sol. Phys., 162, 357

Casini R., Judge P.G., 1999, Spectral Lines for Polarization Measurements of the Coronal Magnetic Field. II. Consistent Treatment of the Stokes Vector forMagnetic-Dipole Transitions, ApJ, 522, 524

Charvin, P., 1965, Étude de la polarisation des raies interdites de la couronne solaire. Application au cas de la raie verte lambda 5303, Annales d'Astrophysique, 28, 877

Charvin, P., 1971, Experimental Study of the Orientation of Magnetic Fields in the Corona, in Solar Magnetic Fields. Symposium no. 43, held at the College de France Paris, France, August 31 to September 4, 1970. Edited by Robert Howard. International Astronomical Union. Symposium no. 43, Dordrecht, Reidel, p.580

Cormack, A.M., 1963, Representation of a function by its line integrals, with some radiological applications, J. Appl. Phys., 34, 2722

Cormack, A.M., 1964, Representation of a function by its line integrals, with some radiological applications 2, J. Appl. Phys., 35, 2908 
Cuperman, S., Ofman, L., Semel, M., 1990, Determination of force-free magnetic fields above the photosphere using three-component boundary conditions: moderately nonlinear case, A\&A, 230, 193

Davila, J.M., 1994, Solar tomography, ApJ, 423, 871

Demoulin, P., Cuperman, S., Semel, M., 1992, Determination of force-free magnetic fields above the photosphere using three-component boundary conditions. II - Analysis and minimization of scale-related growing modes and of computational induced singularities, A\&A, 263, 351

Eddy, John A., McKim Malville, J., 1967, Observations of the Emission Lines of Fe XIII during the Solar Eclipse of may 30, 1965, ApJ, 150, 289

Edlén, Bengt, 1943, Die Deutung der Emissionslinien im Spektrum der Sonnenkorona, Zeitschrift für Astrophysik, 22, 30

Erdelyi, R., 2004, Coronal heating: Heating in the solar atmosphere, Astronomy \& Geophysics, 45(4), 4.34

Esser, R., Brickhouse, N.S., Habbal, S.R., Altrock, R.C., Hudson, H.S., 1995, Using Fe X $6374 \AA$ and Fe XIV $5303 \AA$ spectral line intensities to study the effect of line of sight integration on coronal temperature inferences, JGR, 100, 19829

Flyer, N., Fornberg, B., Thomas, S., Low, B.C., 2004, Magnetic Field Confinement in the Solar Corona. I. Force-free Magnetic Fields, ApJ, 606, 1210

Frazin, R.A., 2000, Tomography of the Solar Corona. I. A Robust, Regularized, Positive Estimation Method, ApJ, 530, 1026

Frazin, R.A., Janzen, P., 2002, Tomography of the Solar Corona. II. Robust, Regularized, Positive Estimation of the Three-dimensional Electron Density Distribution from LASCO-C2 Polarized White-Light Images, ApJ, 570, 408

Fuchs, G., and Pickalov, V., 1998, Vector and scalar tomography on fusion plasmas using Hamiltonian and variational methods, Plasma Phys. Control. Fusion, 40, 91

Gary, G.A., Moore, R.L., Hagyard, M.J., Haisch, B.M., 1987, Nonpotential features observed in the magnetic field of an active region, ApJ, 314, 782

Gary, G.A., 2001, Plasma beta above a solar active region: rethinking the paradigm, Sol. Phys., 203, 71

Gauss, C.F., 1839, Allgemeine Theorie des Erdmagnetismus, Resultate aus den Beobachtungen des magnetischen Vereins im Jahre 1838, 1.

Golnev, V.Z, Pariisky, Y.N., Soboleva, N.S., 1969, Izv. Glavnoi Astron. Obs. in Pulkovo, 23,22

Gordon, R., Bender, R., and Herman, G.T., 1970, Algebraic reconstruction techniques (ART) for the three-dimensional electron microscopy and X-ray photography, J. Theor. Biol., 29, 471 
Grotrian, W., 1939, Sonne und Ionosphäre, Naturwissenschaften, No 34, 569

Guhathakurta, M., Rottman, G.J., Fisher, R.R., Orrall, F.Q., Altrock, R.C., 1992, Coronal density and temperature structure from coordinated observations associated with the total solar eclipse of 1988 March 18, ApJ, 388, 633

Habbal, S.R., Woo, R., Arnaud, J., 2001, On the predominance of the radial component of the magnetic field in the solar corona, ApJ, 558, 852

Hagyard, M.J., Pevtsov, A.A., 1999, Studies of Solar Helicity Using Vector Magnetograms, Sol. Phys., 189, 25

Hanke, M., 1996, Limitations of the L-curve method in ill-posed problems, BIT, 36, 287

Hanle, W., 1924, Über magnetische Beeinflussung der Polarisation der Resonanzfluoreszenz, Z. Phys., 30, 93

Hansen, P.C., 1992, Analysis of discrete ill-posed problems by means of the L-curve, SIAM Review, 34, 561

Hansen, P.C., O'Leary, P.O., 1993, The use of the L-curve in the regularization of discrete ill-posed problems, SIAM J. Sci. Comput., 14, 1487

Herman, G.T., 1980, Image Reconstruction from Projections: The fundamentals of computerized Tomography, New York: Academic Press, 1980

Hounsfield, G.N., 1972, A method of and apparatus for examination of a body by radiation such as X-ray or gamma radiation, Patent Specification 1283915

House L.L., 1974, The theory of the polarization of coronal forbidden lines, Pub.Astron.Soc.Pacific 86, 490

House, L.L., 1977, Coronal emission-line polarization from the statistical equilibrium of magnetic sublevels. I - Fe XIII, ApJ, 214, 632

House, L.L., Querfeld, C.W., Rees, D.E., 1982, Coronal emission-line polarization from the statistical equilibrium of magnetic sublevels. II - Fe XIV 5303 A, ApJ, 255, 753

Howard, J., 1996, Vector tomography applications in plasma diagnostics, Plasma Phys. Control. Fusion, 38, 489

Van de Hulst, H.C., 1950, The electron density of the solar corona, Bulletin of the Astronomical Institute of the Netherlands, 11, 135

Inhester, B., Stenborg, G., Schwenn, R., Srivastava, N., Podlipnik, B., 1999, The Rotation of the Fe XIV Solar Corona During the Recent Solar Activity Minimum, Space Science Reviews, 87, 211

Jacobs, V.L., Davis, J., Kepple, P.C., and Blake, M., 1977, The influence of autoionization accompanied by excitation on dielectronic recombination and ionization equilibrium, ApJ, 211, 605 
Jensen, E.A., Bird, M.K., Asmar, S.W., Anderson, J.D., Russell, C.T., 2005, The Cassini Solar Faraday Rotation experiment, 35th COSPAR Scientific Assembly, held 18 - 25 July 2004, in Paris, France., p.1530

Jiao, L., McClymont, A.N., Mikić, Z., 1997, Reconstruction of the Three-Dimensional Coronal Magnetic Field, Sol. Phys., 174, 311

Kak, A.C., Slaney, M., 1987, Principles of computerized tomographic imaging, IEEE Press, IEEE order number: PP02071

Krall, K.R., Smith, J.B., Jr., Hagyard, M.J., West, E.A., Cumings, N.P., 1982, Vector magnetic field evolution, energy storage, and associated photospheric velocity shear within a flare-productive active region, Sol. Phys., 79, 59

Lamb, F.K., ter Haar, D., 1971, The interaction of atoms with polarised light, Phys. Rep., 2,253

Lanczos, C., 1961, Linear differential operators, London: Van Nostrand, 1961

Landi Degl'Innocenti, E., Landi Degl'Innocenti, M., 1973, A Perturbative Solution of the Transfer Equations for the Stokes Parameters in a Magnetic Field, Sol. Phys., 31, 299

Landi Degl'Innocenti, E., 1982, The determination of vector magnetic fields in prominences from the observations of the Stokes profiles in the D3 line of helium, Sol. Phys., 79,291

Landi Degl'Innocenti, E., 1984, Polarization in spectral lines. III - Resonance polarization in the non-magnetic, collisionless regime, Sol. Phys., 91, 1

Landi Degl'Innocenti, E., Landolfi, M., 2004, Polarization in spectral lines, Dordrecht: Kluwer Academic Publishers, 2004

Landman, D.A., 1975, Proton collisional excitation in the ground configurations of $\mathrm{Fe}^{+12}$ and $\mathrm{Fe}^{+13}$. III - Transitions between magnetic sublevels, A\&A, 43, 285

Lawson, C.L., Hanson, R.J., 1974, Solving least squares problems, Prentice-Hall: Englewood Cliffs, 1974

Levine, R.H., 1976, Evidence for opposed currents in active region loops, Sol.Phys., 46, 159

Lin, H., Penn, M.J., Tomczyk, S., 2000, A new precise measurement of the coronal magnetic field strength, ApJ, 541, 83

Lin, H., Kuhn, J.R., Coulter, R., 2004, Coronal Magnetic Field Measurements ApJ, 613, 177

Lyot B., 1939, The study of the solar corona and prominences without eclipses (George Darwin Lecture, 1939), MNRAS, 99, 580

Mandrini, C.H., Demoulin, P., Klimchuk, J.A., 2000, Magnetic field and plasma scaling laws: their implications for coronal heating models, ApJ, 530, 999 
Miller, K., 1970, Least squares methods for ill-posed problems with a prescribed bound, SIAM J. Math. Anal., 1, 52

Mitchel, A.C.G., Zemansky, M.W., 1934, Resonance radiation and excited atoms, Cambridge University Press, 1934

Mogilevskii, E.I., Nikol'skii, G.M., and Nikol'skaya, K.I., 1960, The polarization of coronal emission lines, Astron. Zh., 37, 236

Natterer, F., 1986, The mathematics of computerized tomography, Stuttgart: Teubner, 1986

Newkirk G.,Jr., Dupree R.G., Schmahl E.J., 1970, Magnetic Fields and the Structure of the Solar Corona. II: Observations of the 12 November 1966 Solar Corona, Sol. Phys., 15,15

Norton, S.J., 1989, Tomographic reconstruction of 2-D vector fields: application to flow imaging, Geophys. J., 97, 161

Osman, N.F., Prince, J.L., 1997, 3-D Vector Tomography on Bounded Domains, http://citeseer.ist.psu.edu/295203.html

Pecker, Ch., Thomas, R. N., 1962, Excitation of the red and green coronal lines, Annales d'Astrophysique, 25, 100

Petrini, D., 1970, The electron exitation rate of the green coronal line $5303 \AA$, A\&A, 9, 392

Phillips, D.L., 1962, A technique for the numerical solution of certain integral equations of the first kind, J. Assoc. Comput. Mach., 9, 84

Querfeld, C.W., Elmore, D.E., 1976, Observation of polarization in FeXIII 10747 Å coronal emission line, Bull. AAS, 8, 368

Querfeld, C.W., Smartt, R.N., 1984, Comparison of coronal emission-line structure and polarization, Sol. Phys., 91, 299

Querfeld, C.W., 1982, The formation and interpretation of the Fe XIII 10747 A coronal emission line, ApJ, 255, 764

Radon, J., 1917, Über die Bestimmung von Funktionen durch ihre Integralwerte längs gewisser Mannigfaltigkeiten, Berichte Sächsische Akademie der Wissenschaften, 69, 262

Raju, K.P., Desai, J.N., Chandrasekhar, T. \& Ashok, N.M., 1991, The excitation mechanism of Fe XIV 5303 A line in the inner regions of solar corona, J. Astrophys. Astr., 12,311

Sahal-Brechot, S., 1974a, Role of Collisions in the Polarization Rate of the Forbidden Emission Lines of the Solar Corona. I. Depolarization by Proton Impact. Application to the Green Line of Fe XIV and to the Infrared Lines of Fe XIII, A\&A, 32, 147 
Sahal-Brechot, S., 1974b, Role of collisions in the polarization degree of the forbidden emission lines of the Solar Corona. II - Depolarization by electron impact and calculation of the polarization degree of the Green line of Fe XIV, A\&A, 36, 355

Sahal-Brechot, S., 1977, Calculation of the polarization degree of the infrared lines of Fe XIII of the solar corona, ApJ, 213, 887

Sakurai, T., 1981, Calculation of force-free magnetic field with non-constant $\alpha$, Sol. Phys., 69, 343

Sakurai, T., 1989, Computational modeling of magnetic fields in solar active regions, Space Science Rev., 51, 11

Schatten, K.H., Wilcox, J.M., \& Ness, N.F., 1969, A model of interplanetary and coronal magnetic fields, Sol. Phys., 6, 442

Scherrer, P.H., Bogart, R.S., Bush, R. I., Hoeksema, J. T., Kosovichev, A. G., Schou, J., Rosenberg, W., Springer, L., Tarbell, T. D., Title, A., Wolfson, C. J., Zayer, I., MDI Engineering Team, The Solar Oscillations Investigation - Michelson Doppler Imager, Sol. Phys., 162, 129

Schmahl, E.J., Kundu, M.R., Strong, K.T., Bentley, R.D., Smith, J.B., Jr., Krall, K.R., 1982, Active region magnetic fields inferred from simultaneous VLA microwave maps, X-ray spectroheliograms, and magnetograms, Sol. Phys., 80, 233

Schwenn, R., et al., 1997, First View of the Extended Green-Line Emission Corona At Solar Activity Minimum Using the Lasco-C1 Coronagraph on SOHO, Sol. Phys., 175, 667

Sparr, G., Strahlen, K., 1998, Vector field tomography, an overview, http://citeseer.ist.psu.edu/sparr98vector.html, http://www.maths.lth.se/matematiklth/personal/gunnar/publications/imaoverview.ps

Stelzried, C.T., Levy, G.S., Sato, T., Rusch, W.V.T., Ohlson, J.E., Schatten, K.H., Wilcox, J.M., 1970, The Quasi-Stationary Coronal Magnetic Field and Electron Density as Determined from a Faraday Rotation Experiment, Sol. Phys., 14, 440

Stern, D.P., 1976, Representation of magnetic fields in space, Rev. Geophys. Space Phys., 14,199

Stix, T.H., 1962, The theory of plasma waves, McGraw-Hill, Inc., 1962

Swindell, W., \& Barrett, H.H., 1977, Computerized tomography: taking sectional x rays, Phys. Today, 30(Dec), 32

Swings, P., 1943, Edln's Identification of the Coronal Lines with Forbidden Lines of Fe X, XI, XIII, XIV, XV; Ni XII, XIII, XV, XVI; Ca XII, XIII, XV; A X, XIV, ApJ, 98, 116

Tikhonov, A.N., 1963, Solution of incorrectly formulated problems and the regularization method, Soviet Math. Dokl., 4, 1035 
Trujillo Bueno, J., 2000, Atomic polarization and the Hanle effect, ASP Conference Series, 236, 161

Ulmschneider, P., 1998, Heating of Chromospheres and Coronae, in Vial et al. (eds), Space Solar Physics, Lecture Notes in Physics, 507, 77.

van Vleck, J.H., 1925, On the Quantum Theory of the Polarization of Resonance Radiation in Magnetic Fields, Proc. Nat. Acad. Sci., 11, 612

Varshalovich, D.A., Moskalev, A.N., Khersonskii, V.K., 1989, Quantum theory of angular momentum: irreducible tensors, spherical harmonics, vectors coupling coefficients, $3 \mathrm{nj}$ symbols, Singapore: World Scientific, 1989

Wang, Y.-M., et al., 1997, The green line corona and its relation to the photospheric magnetic field, ApJ, 485, 419

Weatland, M.S., 2004, Parallel construction of nonlinear force-free fields, Sol. Phys., 222, 247

Wiegelmann, T., 2004, Optimization code with weighting function for the reconstruction of coronal magnetic fields, Sol. Phys., 219, 87

Wilson, D.C., 1976, The three dimensional solar corona. A coronal streamer, Ph.D. Thesis, Colorado Univ. National Center for Atmospheric Research, Boulder, CO

Withbroe, G.L., 1971, The Chemical Composition of the Photosphere and the Corona, in The Menzel Symposium on Solar Physics, Atomic Spectra, and Gaseous Nebulae, Proceedings of a Symposium held in honor of the contributions made by Donald $\mathrm{H}$. Menzel, 8-9 April, 1971 at the Harvard College Observatory, Cambridge, MA. Edited by Katharine B. Gebbie. National Bureau of Standards Special Publication 353. Washington, DC: U.S. Government Printing Office, 1971, p.127

Zare, R.N., 1988, Angular momentum, USA: John Wiley \& Sons, Inc, 1988

Zeeman, P., 1897, On the Influence of Magnetism on the Nature of the Light Emitted by a Substance, ApJ, 5, 332

Zidowitz, Stephan, 1999, Coronal structure of the Whole Sun Month: A tomographic reconstruction, Journal of Geophys. Research, 104, 9727

Zirker, J.B., 1993, Coronal heating, Sol. Phys., 148, 43 



\section{Acknowledgements}

The presented work was performed in the Max-Planck Institute for Solar System Research and was supported by the International Max-Planck Research School on Physical Processes in the Solar System and Beyond at the Universities of Braunschweig and Göttingen. I thank Dr. D. Schmitt, the coordinator of the school, for the organization in the school. Also, I thank him and the Institute's administration staff for the possibility to avoid a much of bureaucracy work.

I am very grateful to Dr. B. Inhester, who proposed the topic for the thesis, for the guidance and helpful discussions during the work. I also thank Dr. T. Wiegelmann and Dr. N.E. Raouafi for the helpful discussions.

Many thanks to Prof. F. Kneer and Prof. E. Marsch for careful reading the thesis and useful remarks.

Thanks to Prof. R. Schwenn and Prof. E. Marsch for the organizing the coronal physics seminars that supported for better understanding of the present state of this branch of physics. Also I thank Prof. F. Kneer for the seminars in solar physics at the Goettingen observatory.

I thank E. A. Jensen for providing me with the information about Cassini's passage beyond the corona.

I also thank my friends and colleagues at MPS for the interesting discussions and nice time we spent together. 



\section{Curriculum Vitae}

Geburt: $\quad$ Am 5.4.1974 in Ruba, Kreis Witebsk, Weißrussland (Belarus)

Eltern: $\quad$ Ivan Kramar

Stepanija Kramar, geb. Dodyk

Staats-

bürgerschaft: Weißrussisch (Belarus)

Schulbildung: 9/1981 bis 7/1991 Schule N28 (Grundschule und Gymnasium), Witebsk

Studium 9/1991 bis 7/1996 Physikstudium an der Physikalisches Fakultät der Staatsuniversität Witebsk.

Diplomarbeit: "The dielectrical properties of the $\mathrm{PbAl}_{1 / 2} \mathrm{Nb}_{1 / 2} \mathrm{O}_{3}-\mathrm{PbIn}_{1 / 2} \mathrm{Nb}_{1 / 2} \mathrm{O}_{3}$ solid solutions"

10/1997 bis 2/2002 Wissenschaftlicher Mitarbeiter des Instituts für Physik der Nationalen Akademie der Wissenschaften von Weißrussland, Minsk

2/2002 bis 3/2005 Doktorarbeit am Max-Planck-Institut für Sonnensystemforschung (früher Aeronomie), Katlenburg-Lindau, und der Universitäts-Sternwarte, Göttingen 